\title{
REPRESENTATIONS OF A SEMISIMPLE LIE GROUP ON A BANACH SPACE. I.
}

\author{
BY \\ HARISH-CHANDRA
}

1. Introduction. During the past few years several authors have studied unitary representations of a Lie group or, more generally, of a locally compact group on a Hilbert space. In the present paper we approach this problem from a somewhat different point of view and do not require that the representation be necessarily unitary. In fact, following a suggestion of Chevalley, we allow the representation space to be a Banach space. But on the other hand we restrict ourselves to the case when the given group is a connected semisimple Lie group and attack the problem by means of a closer study of the corresponding representations of the Lie algebra. Roughly speaking, our plan of investigation can be divided into two separate parts. The first consists in a careful study of infinite-dimensional representations of a semisimple Lie algebra from a purely algebraic point of view. The second step is then to establish a sufficiently close relationship between the representations of the group and those of the algebra so that the information obtained about the latter can be put to use.

This paper is divided into three parts. Part I contains a purely algebraic theorem (Theorem 1) about infinite-dimensional representations of a semisimple Lie algebra. In the special case of a complex semisimple algebra this result had been obtained in a previous paper [8(e)]. Part II is concerned with the second step of the program mentioned above. It has been shown by Gårding [5] that every representation of a Lie group $G$ gives rise in a natural way to a representation of its Lie algebra. However, it turns out that this correspondence between the representations of the group and of its algebra, as it stands, is not very satisfactory for our purpose. This is due to the fact that the Taylor series of an indefinitely differentiable function on $G$ does not necessarily converge to this function. In order to remedy this defect we replace such functions by suitable analytic functions in Gårding's construction and thus obtain what we call well-behaved vectors in the representation space. The remainder of Part II is then devoted to the problem of approximating an arbitrary vector by well-behaved vectors. Theorem 3 deals with this question in a special case which is of particular importance for us.

In Part III we combine the results of Part I with those of Part II and this enables us to derive information about representations of a semisimple group $G$. We introduce the notion of infinitesimal equivalence of two representations and show that in the case of irreducible unitary representations

Received by the editors June 19, 1951. 
this coincides with the usual notion of equivalence. It follows from Theorem 7 that any factor arising from a unitary representation of $G$ is necessarily of type I in the terminology of Murray and von Neumann [14]. (This fact had been conjectured by Mautner [12].) Theorem 9 gives a purely infinitesimal criterion for the existence of a unitary representation which is infinitesimally equivalent to a given irreducible representation. In the last section we give a method for obtaining a large class of representations of $G$.

All the results of this paper, except Theorem 9, were obtained before the end of 1950. Due to various circumstances their full publication has been delayed although these results were announced, along with some others, in a short note $[8(\mathrm{c})]$. In the mean time Godement has obtained alternative proofs for some of them. However the present paper contains only a small proportion of the results which have appeared in a series of notes in the Proceedings of the National Academy [8(d)]. Their proofs will be published in subsequent papers.

I am greatly indebted to Professor Chevalley for reading the original draft of this paper and suggesting a large number of valuable improvements. In fact Part I was almost entirely rewritten by him and in Part II the whole treatment of well-behaved vectors has been very much simplified due to his suggestions. In particular the method of constructing well-behaved functions on a "quasi-nilpotent" group, as given in this paper, is due to Chevalley. My original construction was more complicated.

\section{Part I. Representations of a Semisimple Lie algebra}

2. Preliminary lemmas. Let $k$ be a field of characteristic zero and $\mathfrak{l}$ a Lie algebra over $k$. Let $\mathfrak{t}$ be a subalgebra of $\mathfrak{l}$ and $\sigma$ the representation of $\mathfrak{t}$ on $\mathfrak{l}$ given by $\sigma(X) Y=[X, Y](X \in \mathfrak{l}, Y \in \mathfrak{l})$. Following Koszul [11] we say that $\mathfrak{l}$ is reductive in $\mathfrak{l}$ if $\sigma$ is a semisimple ${ }^{(1)}$ representation. Moreover $\mathfrak{l}$ is called reductive if it is reductive in itself. It is obvious that if $\mathfrak{t}$ is reductive in $\mathfrak{l}$, then $\mathfrak{l}$ is reductive.

Lemma 1. Suppose $\mathfrak{l}$ is reductive. Then $\mathfrak{l}=\mathfrak{c}+\mathfrak{l}^{\prime}, \mathfrak{c} \cap \mathfrak{l}^{\prime}=\{0\}$ where $\mathfrak{c}$ is the center of $\mathfrak{l}$ and $\mathfrak{l}^{\prime}=[\mathfrak{l}, \mathfrak{l}]$ the derived algebra. Moreover $\mathfrak{l}^{\prime}$ is semisimple. A finite-dimensional representation $\rho$ of $\mathfrak{l}$ is semisimple if and only if $\rho(\Gamma)$ is semisimple for every $\Gamma \in \mathfrak{c}$.

Since the adjoint representation of $\mathfrak{l}$ is semisimple, $\mathfrak{l}$ can be written as a direct sum $\sum_{1 \leqq j \leqq m} \mathfrak{l}_{j}$ of simple ideals $\mathfrak{l}_{j}$. Then $\mathfrak{l}_{j}$ is either semisimple or abelian. Suppose $\mathfrak{l}_{j}$ is abelian if $1 \leqq j \leqq m_{1}$, and semisimple if $j>m_{1}\left(0 \leqq m_{1} \leqq m\right)$. Put $\mathfrak{l}^{\prime}=\sum_{j>m_{1}} \mathfrak{l}_{j}$ and $\mathfrak{c}=\sum_{j \leqq m_{1}} \mathfrak{l}_{j}$. Then $\mathfrak{l}^{\prime}$ is semisimple and it is clear that $\mathfrak{c}$ is the kernel of the adjoint representation. Hence $\mathfrak{c}$ is the center and

(1) We use here the terminology of Chevalley [4, Chap. VI]. A linear transformation of a vector space $V$ (of finite dimension) is called semisimple if $V$ is fully reducible under $A$. 


$$
[\mathfrak{l}, \mathfrak{l}]=\sum_{\mathfrak{l} \leqq j \leqq m}\left[\mathfrak{l}_{j}, \mathfrak{l}_{j}\right]=\sum_{j>m_{1}} \mathfrak{l}_{j}=\mathfrak{l}^{\prime} .
$$

This proves the first part. Now $\rho(\mathfrak{l})=\rho(\mathfrak{c})+\rho\left(\mathfrak{l}^{\prime}\right)$. Since $\mathfrak{l}^{\prime}$ is semisimple the same holds for the linear Lie algebra $\rho\left(\mathfrak{l}^{\prime}\right)$. So the center of $\rho\left(\mathfrak{l}^{\prime}\right)$ is $\{0\}$ and therefore $\rho(\mathfrak{c})$ is the center of $\rho(\mathfrak{l})$. Our second assertion now follows from a result of Jacobson [10].

Lemma 2. Suppose $\mathfrak{l}$ is reductive in $\mathfrak{l}$ and $\rho$ is a finite-dimensional semisimple representation of $\mathfrak{l}$. Then $\rho$ induces a semisimple representation of $\mathfrak{l}$.

Let $\mathfrak{D}$ be the center of $\mathfrak{t}$. Since $\mathfrak{t}$ is reductive, $\rho(\mathfrak{D})$ is the center of $\rho(\mathfrak{t})$. Put $\mathfrak{l}_{1}=\rho(\mathfrak{l}), \mathfrak{l}_{1}=\rho(\mathfrak{l})$, and $\mathfrak{D}_{1}=\rho(\mathfrak{D})$. Since $\mathfrak{l}$ is reductive in $\mathfrak{l}$, it is clear that $\mathfrak{l}_{1}$ is reductive in $\mathfrak{l}_{1}$. Moreover since $\rho$ is semisimple, $\mathfrak{l}_{1}$ is reductive (see Jacobson [10]). Let $\mathfrak{c}_{1}$ be the center and $\mathfrak{l}_{1}^{\prime}$ the derived algebra of $\mathfrak{l}_{1}$. Let $\sigma$ denote the adjoint representation of $\mathfrak{l}_{1}$. Then we know from the above lemma that $\sigma(D)$ is semisimple for any $D \in \mathfrak{D}_{1}$. Choose an abelian subalgebra $\mathfrak{h}_{1}$ of $\mathfrak{l}_{1}$ such that $\mathfrak{h}_{1} \supset \mathfrak{D}_{1}, \sigma(H)$ is semisimple for all $H \in \mathfrak{h}_{1}$, and $\mathfrak{h}_{1}$ is not contained in a larger such algebra. Then $\mathfrak{h}_{1} \supset \mathfrak{c}_{1}$, and it is clear that $\mathfrak{l}_{1}^{\prime} \cap \mathfrak{h}_{1}$ is a Cartan subalgebra of the semisimple algebra $\mathfrak{l}_{1}^{\prime}$. Now if $\phi$ is a semisimple representation of $\mathfrak{l}_{1}$ it follows from the above lemma that $\phi(\Gamma)$ is semisimple for all $\Gamma \in c_{1}$. Moreover $\phi(H)$ is semisimple for every $H \in \mathfrak{l}_{1}^{\prime} \cap \mathfrak{h}_{1}$, since $\mathfrak{l}_{1}^{\prime} \cap \mathfrak{h}_{1}$ is a Cartan subalgebra of the semisimple algebra $\mathfrak{l}_{1}^{\prime}$. Hence $\phi(H)$ is semisimple for all $H \in \mathfrak{h}_{1}$ and therefore in particular for $H \in \mathfrak{D}_{1}$. Therefore, again by the above lemma, the representation of $\boldsymbol{t}_{1}$ defined by $\phi$ is semisimple. In particular if we take for $\phi$ the representation $\phi: \rho(X) \rightarrow \rho(X)(X \in \mathfrak{l})$, we get the required result.

Let $R$ and $C$ be the fields of real and complex numbers respectively and $(-1)^{1 / 2}$ a fixed square root of -1 in $C$. Let $g_{0}$ be a semisimple Lie algebra over $R$ and $\mathrm{g}$ its complexification. Let $X \rightarrow \mathrm{ad} X(X \in \mathfrak{g})$ denote the adjoint representation of $\mathfrak{g}$. Put $B(X, Y)=\operatorname{sp}(\operatorname{ad} X$ ad $Y)$. A real form $\mathrm{g}_{k}$ of $\mathfrak{g}$ is called compact if $B(X, X)<0$ for all $X \in \mathrm{g}_{k}(X \neq 0)$. It is well known (see Cartan [3] and also Mostow [13]) that there exists a compact real form $\mathrm{g}_{k}$ and an automorphism $\theta$ of order 2 of $\mathfrak{g}$ with the following properties: $\theta \mathfrak{g}_{0} \subset \mathfrak{g}_{0}, \theta \mathfrak{g}_{k} \subset \mathfrak{g}_{k}$ and

$$
\mathfrak{g}_{0}=\mathfrak{f}_{0}+\mathfrak{p}_{0}, \quad \mathfrak{g}_{k}=\mathfrak{f}_{0}+(-1)^{1 / 2} \mathfrak{p}_{0}
$$

where $\mathfrak{f}_{0}$ is the set of all $X \in \mathfrak{g}_{0}$ such that $\theta X=X$ and $\mathfrak{p}_{0}$ the set of all $Y \in \mathfrak{g}_{0}$ such that $\theta Y=-Y$. Let $\mathfrak{t}$ and $\mathfrak{p}$ be the subspaces of $\mathfrak{g}$ spanned by $\mathfrak{f}_{0}$ and $\mathfrak{p}_{0}$ respectively over $C$. Since $\theta$ is an automorphism, $\theta([X, Y])=[\theta X, \theta Y]$ $(X, Y \in \mathfrak{g})$ and from this it follows immediately that

$$
[\mathfrak{f}, \mathfrak{f}] \subset \mathfrak{t}, \quad[\mathfrak{f}, \mathfrak{p}] \subset \mathfrak{p}, \quad[\mathfrak{p}, \mathfrak{p}] \subset \mathfrak{t} .
$$

Hence in particular $\mathfrak{l}$ is a subalgebra of $\mathfrak{g}$.

LEMMA 3. $\mathfrak{t}$ is reductive in $\mathfrak{g}$. 
Let $G$ be the component of identity of the adjoint group of $g_{0}$ and $K$ the analytic subgroup of $G$ corresponding to $\mathfrak{P}_{0}$. Then it is known (see Mostow [13]) that $K$ is compact. From this it follows immediately that $\mathfrak{t}_{0}$ is reductive in $\mathfrak{g}_{0}$ and therefore $\mathfrak{l}$ in $\mathfrak{g}$.

Let $\mathfrak{h}_{\mathfrak{p}_{0}}$ be an abelian subalgebra of $\mathfrak{g}_{0}$ contained in $\mathfrak{p}_{0}$ and having the maximal possible dimension. Extend $\mathfrak{h}_{\mathfrak{p}_{0}}$ to a maximal abelian subalgebra $\mathfrak{h}_{0}$ of $\mathfrak{g}_{0}$. Let $X \in \mathfrak{h}_{0}$. Then if $Y \in \mathfrak{h}_{\mathfrak{p}_{0}},[\theta X, Y]=-[\theta X, \theta Y]=-\theta([X, Y])=0$. Hence $X-\theta X$ commutes with all elements in $\mathfrak{h}_{\mathfrak{p}_{0}}$. But $X-\theta X \in \mathfrak{p}_{0}$ and therefore in view of our choice of $\mathfrak{h}_{\mathfrak{p}_{0}}, X-\theta X \in \mathfrak{h}_{\mathfrak{p}_{0}}$. This proves that $\theta \mathfrak{h}_{0}=\mathfrak{h}_{0}$ and therefore $\mathfrak{h}_{0}=\mathfrak{h}_{\mathfrak{t}_{0}}+\mathfrak{h}_{\mathfrak{p}_{0}}$ where $\mathfrak{h}_{\mathfrak{t}_{0}}=\mathfrak{h}_{0} \cap \mathfrak{l}_{0}$. Since $\mathfrak{g}_{k}=\mathfrak{l}_{0}+(-1)^{1 / 2} \mathfrak{p}_{0}$ is compact, ad $X$ is semisimple for every $X \in \mathfrak{l}_{0} \cup_{\mathfrak{p}_{0}}$. Therefore if $\mathfrak{h}$ is the subspace of $\mathfrak{g}$ spanned by $\mathfrak{h}_{0}, \mathfrak{h}$ is a Cartan subalgebra of $\mathfrak{g}$. Put $\mathfrak{h}_{\mathfrak{p}}=\mathfrak{p} \cap \mathfrak{h}, \mathfrak{h}_{\mathfrak{f}}=\mathfrak{l} \cap \mathfrak{h}$. Let $H_{1}, \cdots, H_{l}$ be a base for $\mathfrak{h}_{0}$ (over $R$ ) such that $H_{1}, \cdots, H_{m}$ is a base for $\mathfrak{h}_{\mathfrak{p}_{0}}$ and $H_{m+1}, \cdots, H_{l}$ is a base for $\mathfrak{h}_{\mathfrak{t}_{0}}$. Put $H_{i}^{*}=H_{i}$ or $(-1)^{1 / 2} H_{i}$ according as $i \leqq m$ or $i>m$ and let $\mathfrak{h}^{*}=\mathfrak{h}_{\mathfrak{p}_{0}}+(-1)^{1 / 2} \mathfrak{h}_{\mathfrak{t}_{0}}$. For any linear function $\lambda$ on $\mathfrak{h}$ let $H_{\lambda}$ denote the unique element in $\mathfrak{h}$ such that

$$
B\left(H_{\lambda}, H\right)=\lambda(H)
$$

We say that $\lambda$ is real if $H_{\lambda} \in \mathfrak{h}^{*}$. Now let $H=\sum_{i=1}^{l} e_{i} H_{i}^{*}\left(c_{i} \in R\right)$ be any element in $\mathfrak{h}^{*}$. We say that $H>0$ if $H \neq 0$ and $c_{j}>0$ where $j$ is the least index such that $c_{j} \neq 0$. If $\lambda$ and $\mu$ are two linear functions on $\mathfrak{h}$ such that $\lambda-\mu$ is real, then we write $\lambda>\mu$ or $\mu<\lambda$ if $H_{\lambda-\mu}=H_{\lambda}-H_{\mu}>0$. Moreover we denote by $\theta \lambda$ the linear function $H \rightarrow \lambda(\theta H)(H \in \mathfrak{h})$.

It is known that every root $\alpha$ of $\mathfrak{g}$ with respect to $\mathfrak{h}$ is a real linear function and if $\alpha$ is a root $\theta \alpha$ is also a root. For every root $\alpha$ choose an element $X_{\alpha} \in \mathrm{g}$ such that $X_{\alpha} \neq 0$ and $\left[H, X_{\alpha}\right]=\alpha(H) X_{\alpha}$ for all $H \in \mathfrak{h}$. Let $P$ be the set of all roots $\alpha>0$. Define the subsets $P_{+}$and $P_{-}$of $P$ as follows. A root $\alpha \in P$ belongs to $P_{+}$or $P_{-}$according as $\theta \alpha \neq \alpha$ or $\theta \alpha=\alpha$. It can be shown (see Iwasawa [9]) that $\theta \alpha<0$ for $\alpha \in P_{+}$and $H_{\beta} \in \mathfrak{h}_{\mathfrak{t}}$ and $X_{\beta}, X_{-\beta} \in \mathfrak{l}$ for $\beta \in P_{-}$. Moreover $\alpha>\beta$ for any $\alpha \in P_{+}$and $\beta \in P_{-}$. Let $\mathfrak{n}$ be the subspace of $\mathfrak{g}$ spanned by $X_{\alpha}\left(\alpha \in P_{+}\right)$and $\mathfrak{m}$ the subspace spanned by $\mathfrak{h}_{\mathfrak{l}}, X_{\beta}$ and $X_{-\beta}\left(\beta \in P_{-}\right)$. Then $\mathfrak{n}$ is a nilpotent subalgebra of $\mathfrak{g}$. Put $\mathfrak{n}_{0}=\mathfrak{g}_{0} \cap \mathfrak{n}$. I wasawa [9] has proved that $\mathfrak{g}_{0}$ is the direct sum of $\mathfrak{t}_{0}, \mathfrak{h}_{\mathfrak{p}_{0}}$, and $\mathfrak{n}_{0}$.

Lemma 4. The centraliser of $\mathfrak{h}_{\mathfrak{p}}$ in $\mathfrak{g}$ is $\mathfrak{h}_{\mathfrak{p}}+\mathfrak{m}$ and so $\mathfrak{m}$ is the centraliser of $\mathfrak{h}_{\mathfrak{p}}$ in $\mathfrak{f}$. Moreover there exists an element $H \in \mathfrak{h}_{\mathfrak{p}}$ whose centraliser (in $\mathfrak{g}$ ) is exactly $\mathfrak{h}_{\mathfrak{p}}+\mathfrak{m}$. Finally $\mathfrak{m}$ is a subalgebra of $\mathfrak{l}$ and

$$
\operatorname{dim} \mathfrak{l}-\operatorname{dim} \mathfrak{m}=\operatorname{dim} \mathfrak{p}-\operatorname{dim} \mathfrak{h}_{\mathfrak{p}}=\operatorname{dim} \mathfrak{n} .
$$

Let $X=H_{0}+\sum_{\alpha \in P}\left(c_{\alpha} X_{\alpha}+c_{-\alpha} X_{-\alpha}\right)\left(H_{0} \in \mathfrak{h} ; c_{\alpha}, c_{-\alpha} \in C\right)$. Then

$$
[H, X]=\sum_{\alpha \in P}\left(c_{\alpha} \alpha(H) X_{\alpha}-c_{-\alpha} \alpha(H) X_{-\alpha}\right) \quad(H \in \mathfrak{h}) \text {. }
$$

Therefore if $X$ commutes with $\mathfrak{h}_{\mathfrak{p}}, c_{\alpha}=c_{-\alpha}=0$ unless $\alpha$ is identically zero on 
$\mathfrak{h}_{\mathfrak{p}}$. But $\alpha$ is zero on $\mathfrak{h}_{\mathfrak{p}}$ if and only if $\alpha=\theta \alpha$. This proves that $\mathfrak{h}_{\mathfrak{p}}+\mathfrak{m}$ is the centraliser of $\mathfrak{h}_{\mathfrak{p}}$ in $\mathfrak{g}$. Choose $H \in \mathfrak{h}_{\mathfrak{p}}$ such that $\alpha(H) \neq 0$ for $\alpha \in P_{+}$. Then it is clear from the above argument that the centraliser of $H$ is $\mathfrak{h}_{\mathfrak{p}}+\mathfrak{m}$.

If $\alpha \in P_{+}, \theta \alpha<0$ and so $-\theta \alpha>0$. Hence $-\theta \alpha \neq-\alpha$ and this shows that $-\theta \alpha \in P_{+}$. The mapping $\alpha \rightarrow-\theta \alpha\left(\alpha \in P_{+}\right)$therefore defines a permutation of order 2 on $P_{+}$. Thus when $\alpha$ runs through $P_{+}, \theta \alpha$ runs through the set $\left\{-\alpha ; \alpha \in P_{+}\right\}$. Since $\theta X_{\alpha}$ and $X_{\theta \alpha}$ differ only by a nonzero constant factor, it follows that $X_{\alpha}, \theta X_{\alpha}\left(\alpha \in P_{+}\right)$are linearly independent modulo $\mathfrak{h}_{\mathfrak{p}}+\mathfrak{m}$. The same therefore holds for $X_{\alpha}-\theta X_{\alpha}, X_{\alpha}+\theta X_{\alpha}\left(\alpha \in P_{+}\right)$. Let $q$ $=\sum_{\alpha \in P_{+}} C\left(X_{\alpha}-\theta X_{\alpha}\right)$ and $\mathfrak{l}=\sum_{\alpha \in P_{+}} C\left(X_{\alpha}+\theta X_{\alpha}\right)$. Then $\mathfrak{q} \subset \mathfrak{p}$ and $\mathfrak{l} \subset \mathfrak{l}$ and $\mathfrak{q}+\mathfrak{l}+\mathfrak{h}_{\mathfrak{p}}+\mathfrak{m}=\mathfrak{g}=\mathfrak{p}+\mathfrak{l}$. Hence $\mathfrak{q}+\mathfrak{h}_{\mathfrak{p}}=\mathfrak{p}, \mathfrak{l}+\mathfrak{m}=\mathfrak{l}$, and if $q$ is the number of roots in $P_{+}$, it is clear that $\operatorname{dim} \mathfrak{n}=\mathfrak{q}=\operatorname{dim} q=\operatorname{dim} \mathfrak{p}-\operatorname{dim} \mathfrak{h}_{\mathfrak{p}}$. Similarly $q=\operatorname{dim} \mathfrak{l}=\operatorname{dim} \mathfrak{t}-\operatorname{dim} \mathfrak{m}$.

Since $\mathfrak{m}$ is the centraliser of $\mathfrak{h}_{\mathfrak{p}}$ in $\mathfrak{l}, \mathfrak{m}$ is a subalgebra of $\mathfrak{t}$.

Lemma $5 . \mathfrak{m}$ is reductive both in $\mathfrak{l}$ and in $\mathfrak{g}$ and $[\mathfrak{m}, \mathfrak{n}] \subset \mathfrak{n}$. Furthermore if $\sigma$ is the adjoint representation of the algebra $\mathfrak{h}_{\mathfrak{p}}+\mathfrak{n}$, then $\mathrm{sp} \sigma(H)=\sum_{\alpha \in P} \alpha(H)$ $\left(H \in \mathfrak{h}_{\mathfrak{p}}\right)$.

Put $\mathfrak{m}_{0}=\mathfrak{m} \cap \mathfrak{g}_{0}$ and define $G$ and $K$ as in the proof of Lemma 3. Let $M$ be the set of all $x \in K$ such that $x H=H\left(H \in \mathfrak{h}_{\mathfrak{p}_{0}}\right)$. Clearly $M$ is a closed subgroup of $K$ and so it is compact. Moreover from Lemma $4 \mathrm{~m}_{0}$ is the subalgebra of $\mathfrak{l}_{0}$ corresponding to the component of identity of $M$. Hence $\mathfrak{m}_{0}$ is reductive in $\mathfrak{l}_{0}$ and $\mathfrak{g}_{0}$ and therefore the same is true of $\mathfrak{m}$ in $\mathfrak{f}$ and $\mathfrak{g}$.

Let $\alpha \in P_{+}$and $\beta \in P_{-}$. Then $\alpha+\beta$ and $\alpha-\beta$ are both greater than 0 . Moreover $\theta(\alpha+\beta)=\theta \alpha+\beta \neq \alpha+\beta$ since $\alpha \neq \theta \alpha$. Similarly $\theta(\alpha-\beta) \neq \alpha-\beta$. This shows that $\left[X_{\alpha}, X_{\beta}\right]$ and $\left[X_{\alpha}, X_{-\beta}\right]$ are both in $\mathfrak{\mathfrak { n }}$. Since $[\mathfrak{h}, \mathfrak{n}] \subset \mathfrak{n}$, we conclude that $[\mathfrak{m}, \mathfrak{n}] \subset \mathfrak{n}$.

It is clear that sp $\sigma(H)=\sum_{\alpha \in P_{+}} \alpha(H)\left(H \in \mathfrak{h}_{\mathfrak{p}}\right)$. Since $\alpha$ is zero on $\mathfrak{h}_{\mathfrak{p}}$ if $\alpha \in P_{-}$, it follows that sp $\sigma(H)=\sum_{\alpha \in P} \alpha(H)$.

Let $\rho$ be a representation of $\mathfrak{t}$ on a vector space $V$ whose dimension need not be finite. A subspace $W$ of $V$ will be called $\rho$-stable if $\rho(X) W \subset W$ for all $X \in \mathfrak{l}$. Let $\rho_{W}$ denote the representation of $\mathfrak{t}$ induced on a $\rho$-stable subspace $W$. We shall call $\rho_{W}$ simple (semisimple) if $\operatorname{dim} W<\infty$ and $\rho_{W}$ is simple (semisimple) in the usual sense (see Chevalley [4, Chap VI]). Also we then say that $W$ is a simple (semisimple) subspace of $V$.

Let $\Omega$ denote the set of all (equivalence) classes of simple representations of $\mathfrak{t}$. For any $\mathfrak{D} \in \Omega$ we denote by $V_{\mathfrak{D}}$ the sum of all $\rho$-stable subspaces $W$ of $V$ such that $\rho_{W} \in \mathfrak{D}$. $\left(V_{\mathbb{D}}=\{0\}\right.$ if no such $W$ exists.)

Lemma 6. If $W$ is any $\rho$-stable subspace of $V$, then

$$
W \cap\left(\sum_{\mathfrak{D} \in \Omega} V_{\mathfrak{D}}\right)=\sum_{\mathbb{D}} W \cap V_{\mathfrak{D}} .
$$

Moreover the sum in $\sum_{D \in \Omega} V_{D}$ is direct. 
For any $x \in \sum_{\mathscr{D} \in \Omega} V_{\mathfrak{D}}$ let $V_{x}$ be the smallest $\rho$-stable subspace containing $x$. It is clear that $V_{x}$ is contained in the sum of a finite number of simple spaces. Hence $V_{x}$ is semisimple (see Chevalley [4, Chap. VI]) and therefore $V_{x}=\sum_{D} V_{x} \cap V_{D}$. Applying this result to the elements of $W \cap\left(\sum_{D \in \Omega} V_{D}\right)$ we get the first statement of the lemma. Let $\Omega_{1}$ be a subset of $\Omega$. Then for any $x \in \sum \sum_{\mathscr{N} \in \Omega_{1}} V_{\mathscr{D}}, V_{x}$ is contained in the sum of a finite number of simple spaces $W^{\prime}$ such that the class of $\rho_{W^{\prime}}$ lies in $\Omega_{1}$. Hence it follows (see Chevalley loc. cit.) that every simple component of $\rho_{V x}$ belongs to a class in $\Omega_{1}$ and therefore $V_{x} \cap V_{\mathscr{D}}=\{0\}$ if $\mathfrak{D} \in \Omega_{1}$. This proves the directness of the sum $\sum \sum_{\mathbb{E} \in \Omega} V_{\mathfrak{D}}$.

An element $x \in V$ will be said to transform (under $\rho$ ) according to $\mathfrak{D}$ if $x \in V_{D}$ and we shall say that the representation $\rho$ is quasi-semisimple if $V=\sum_{\mathscr{D} \in \Omega} V_{\mathfrak{D}}$. In order that this be the case it is necessary and sufficient that every element $x \in V$ should belong to some semisimple subspace $W$ of $V$. The following lemma is well known (see Godement [7(b), p. 102]).

Lemma 7. Assume that $\rho$ is quasi-semisimple. Let $V_{0}$ be the space of all $x \in V$ such that $\rho(X) x=0$ for all $X \in \mathfrak{f}$ and let $V_{1}$ be the space spanned by all elements $\rho(X) x(X \in \mathfrak{f}, x \in V)$. Then $V$ is the direct sum of $V_{0}$ and $V_{1}$.

Let $\mathfrak{D}_{0}$ be the class of the simple zero representation of degree 1 of $\mathfrak{t}$. Then $V_{0}=V_{D_{0}}$ and it is clear that $V_{1} \subset \sum_{D \neq D_{0}} V_{D}$ which shows that the sum $V_{0}+V_{1}$ is direct. In order to prove that $V_{0}+V_{1}=V$ it is sufficient to show that if $W$ is a $\rho$-stable subspace of $V$ such that $\rho_{W} \in \mathfrak{D}\left(\mathfrak{D} \neq \mathfrak{D}_{0}\right)$, then $W \subset V_{1}$. The subspace $W^{\prime}$ of $W$ spanned by the elements $\rho(X) x(X \in \mathfrak{l}, x \in W)$ is clearly $\rho$-stable and not equal to $\{0\}$ since $\rho_{W}$ is simple and not in $\mathfrak{D}_{0}$. Hence $W=W^{\prime}$, which proves our assertion.

Let $V$ and $V^{\prime}$ be the spaces of the representations $\rho$ and $\rho^{\prime}$ of $t$. Then the tensor product $V \times V^{\prime}$ is the space of a representation $\rho+\rho^{\prime}$ (the tensor sum of $\rho$ and $\left.\rho^{\prime}\right)$ which is defined as follows: if $X \in \mathfrak{l}, x \in V$, and $x^{\prime} \in V^{\prime}$, then $\left(\left(\rho+\rho^{\prime}\right)(X)\right)\left(x \times x^{\prime}\right)=(\rho(X) x) \times x^{\prime}+x \times\left(\rho\left(X^{\prime}\right) x^{\prime}\right)$.

Lemma 8. Assume that the representations $\rho$ and $\rho^{\prime}$ are quasi-semisimple. Then $\rho+\rho^{\prime}$ is also quasi-semisimple.

Every element of $V \times V^{\prime}$ is contained in a space of the form $W \times W^{\prime}$ where $W$ and $W^{\prime}$ are semisimple subspaces of $V$ and $V^{\prime}$ respectively. Let $\mathrm{c}$ be the center of $\mathfrak{x}$. Since $\rho_{W}$ is semisimple, it follows from Lemma 1 that we can choose a base $\left(x_{1}, \cdots, x_{m}\right)$ in $W$ such that the matrix representing $\rho_{W}(\Gamma)$ $(\Gamma \in \mathfrak{c})$ relative to this base is diagonal. A similar base $\left(x_{1}^{\prime}, \cdots, x_{m^{\prime}}^{\prime}\right)$ can be found for $W^{\prime}$ with respect to $\rho_{W^{\prime}}^{\prime}$. But then it is clear that $\left(\rho+\rho^{\prime}\right)_{W+W^{\prime}}(\Gamma)$ $(\Gamma \in \mathfrak{c})$ is represented by a diagonal matrix relative to the base $x_{i} \times x_{j}^{\prime}$, $1 \leqq i \leqq m ; 1 \leqq j \leqq m^{\prime}$, for $W \times W^{\prime}$. In view of Lemma 1 this shows that $W \times W^{\prime}$ is semisimple under $\rho+\rho^{\prime}$. Hence every element of $V \times V^{\prime}$ is contained in a semisimple subspace and this proves the lemma. 
Let $\mathfrak{B}$ be the universal enveloping $\left({ }^{2}\right)$ algebra of $\mathfrak{g}$ and $\mathfrak{X}$ the subalgebra of $\mathfrak{B}$ generated by $(1, \mathfrak{t})$. Then $\mathfrak{X}$ is the universal enveloping algebra of $\mathfrak{t}$ (see [8(e)]) and there is a natural 1-1 correspondence between representations of $\mathfrak{f}$ and their unique extensions $\left.{ }^{3}\right)$ on $\mathfrak{X}$. It is convenient to identify the representations of $\mathfrak{t}$ and $\mathfrak{X}$ under this correspondence. Let $\pi$ be a representation of $\mathfrak{B}$ on $V$. Then the restriction of $\pi$ on $\mathfrak{t}$ is a representation of $\mathfrak{t}$. For any $\mathfrak{D} \in \Omega$ we define $V_{\mathfrak{D}}$ as the set of all elements of $V$ which transform under $\pi(\mathfrak{l})$ according to $\mathfrak{D}$.

\section{Lemma 9. The subspace $\sum_{\mathbb{D} \in \Omega} V_{\mathfrak{D}}$ is invariant under $\pi(\mathfrak{B})$.}

Let $V_{0}=\sum_{\mathfrak{D} \in \Omega} V_{\mathfrak{D}}$. Since $(1, \mathfrak{g})$ generates $\mathfrak{B}$ it would be sufficient to show that $\pi(\mathfrak{g}) V_{0} \subset V_{0}$. But $\mathfrak{g}=\mathfrak{f}+\mathfrak{p}$ and $V_{0}$ is obviously invariant under $\pi(\mathfrak{f})$. Hence we have only to show that $\pi(\mathfrak{p}) V_{0} \subset V_{0}$. Let $\sigma$ be the representation of $\mathfrak{f}$ on $\mathfrak{p}$ defined by $\sigma(X) Y=[X, Y](X \in \mathfrak{f}, Y \in \mathfrak{p})$ and let $\rho$ be the representation of $\mathfrak{t}$ on $V_{0}$. Consider $\mathfrak{p} \times V_{0}$ and the representation $\sigma+\rho$ induced on it. Since $\mathfrak{t}$ is reductive in $\mathfrak{g}, \sigma$ is semisimple. Moreover for any given $x \in V_{0}$ we can find a $\rho$-stable semisimple subspace $W$ of $V_{0}$ such that $x \in W$. Then $\mathfrak{p} \times W$ is semisimple under the representation $\nu$ induced on it under $\sigma+\rho$ (Lemma 8). Let $\lambda$ be the linear mapping of $p \times W$ into $V$ defined by $\lambda(Y \times w)$ $=\pi(Y) w(Y \in \mathfrak{p}, w \in W)$. Then it is easy to verify that $\pi(X) \lambda=\lambda \nu(X)$. This means that $\lambda$ is a homomorphism of the $\mathfrak{f}$-module $p \times W$ into the $\mathfrak{l}$-module $V$. Since $\mathfrak{p} \times W$ is semisimple the same holds for $\lambda(\mathfrak{p} \times W)$ (see Chevalley [4, Chap. VI $])$. Since $\pi(\mathfrak{p}) x \subset \lambda(\mathfrak{p} \times W)$ it follows that $\pi(\mathfrak{p}) x$ is contained in a semisimple $\mathfrak{t}$-submodule of $V$ and therefore $\pi(\mathfrak{p}) x \subset V_{0}$. This proves the lemma.

Let $V$ be a vector space over $C$ of finite dimension. Then $V$ may be considered as an abelian Lie algebra and the universal enveloping algebra of this Lie algebra is called the symmetric algebra over $V$. We shall denote it by $S(V)$. If $\left\{x_{1}, \cdots, x_{n}\right\}$ is a base for $V$, the monomials, $x_{1}^{e_{1}} x_{2}^{e_{2}} \cdots x_{n}^{e_{n}}\left(e_{i} \geqq 0 ; 1 \leqq i \leqq n\right)$ form a base for $S(V)$ and so $S(V)$ may be regarded as the algebra of polynomials in $x_{1}, \cdots, x_{n}$. For any integer $d \geqq 0$ we denote by $S_{d}(V)$ the subspace of $S(V)$ consisting of all forms in $\left(x_{1}, \cdots, x_{n}\right)$ of degree $d$. It is clear that $S_{d}(V)$ is independent of the particular choice of the base $\left(x_{1}, \cdots, x_{n}\right)$. We call an element $F \in S(V)$ homogeneous of degree $d$ if $F \in S_{d}(V)$.

Let $T$ be an endomorphism of $V$. Then it follows from the structure of $S(V)$ as an algebra of polynomials that $T$ may be extended uniquely to a derivation $d_{T}$ of $S(V)$. If $f \in P\left(x_{1}, \cdots, x_{n}\right)$ where $P$ is a polynomial, then $d_{T} f=\sum_{1 \leqq i \leqq n}\left(\partial P\left(x_{1}, \cdots, x_{n}\right) / \partial x_{i}\right)\left(T x_{i}\right)$. Hence $d_{T}$ maps $S_{d}(V)$ into itself. Moreover the mapping $T \rightarrow d_{T}$ is linear and $\left({ }^{4}\right) d_{\left[T_{1}, T_{2}\right]}=\left[d_{T_{1}}, d_{T_{2}}\right]$.

(2) For a definition of the universal enveloping algebra see [8(a)].

( $\left.{ }^{3}\right)$ Let $\mathfrak{A}$ be an associative algebra with a unit element 1 and $\pi$ a representation of $\mathscr{A}$. We shall always assume that $\pi(1)$ is the identity mapping of the representation space and this condition will be included in the definition of a representation.

(4) Throughout this paper we write $[a, b]=a b-b a$ whenever $a, b$ lie in an associative algebra or are endomorphisms of a vector space. 
Now consider in particular the algebra $S(\mathfrak{g})$. The product of elements of $\mathfrak{g}$ do not have the same meaning in $S(\mathfrak{g})$ as in $\mathfrak{B}$. In order to avoid confusion we shall represent by $\bar{X}$ an element $X \in \mathfrak{g}$ when we want to consider it as an element of $S(\mathfrak{g})$ (this is less cumbersome than introducing different notations for multiplications in $S(\mathfrak{g})$ and in $\mathfrak{B})$. Let $\left(X_{1}, \cdots, X_{n}\right)$ be a base for $\mathfrak{g}$. Define a linear mapping $\lambda$ of $S(\mathfrak{g})$ into $\mathscr{B}$ as follows:

$$
\lambda\left(\bar{X}_{1}^{e_{1}} \cdots \bar{X}_{n}^{e_{n}}\right)=(1 / m !) \sum X_{i_{1}} X_{i_{2}} \cdots X_{i_{m}} \quad\left(0 \leqq e_{i}<\infty, 1 \leqq i \leqq n\right)
$$

where $m=e_{1}+\cdots+e_{n}$ and the summation is over all sequences $\left(i_{1}, \cdots, i_{m}\right)$ which have exactly $e_{j}$ terms equal to $j(1 \leqq j \leqq n)$. It is clear that if $Y_{1}, \cdots, Y_{k}$ are any elements of $\mathfrak{g}$, then

$$
\lambda\left(\bar{Y}_{1} \bar{Y}_{2} \cdots \bar{Y}_{k}\right)=(1 / k !) \sum_{\omega} Y_{\omega(1)} \cdots Y_{\omega(k)}
$$

where the summation is over all permutations $\omega$ of $\{1,2, \cdots, k\}$. This shows that the definition of $\lambda$ is independent of the choice of the base $\left(X_{1}, \cdots, X_{m}\right)$. We know from theorems of [8(a)] that $\lambda$ is a 1-1 linear mapping of $S(\mathfrak{g})$ onto $\mathfrak{B}$. We shall call it the canonical mapping of $S(\mathfrak{g})$ onto $\mathfrak{B}$.

Let $\sigma(X)(X \in \mathfrak{g})$ be the derivation of $S(\mathfrak{g})$ which coincides with ad $X$ on $\mathfrak{g}$. Then $\sigma: X \rightarrow \sigma(X)$ is a representation of $\mathfrak{g}$ on $S(\mathfrak{g})$. Let $\sigma_{\mathfrak{t}}$ be the restriction of $\sigma$ to $\mathrm{t}$.

\section{LEMMA 10. $\sigma_{\mathfrak{t}}$ is a quasi-semisimple representation of $\mathfrak{t}$.}

Let $\sigma_{d}(X)(X \in \mathfrak{g})$ denote the restriction of $\sigma(X)$ on $S_{d}(\mathfrak{g})$. Then $\sigma_{d}$ is a finite-dimensional representation of $\mathfrak{g}$. Since $\mathfrak{g}$ is semisimple and $\mathfrak{t}$ is reductive in $\mathfrak{g}, \sigma_{d}$ induces a semisimple representation of $\mathfrak{l}$ on $S_{d}(\mathfrak{g})$. But $S(\mathfrak{g})=\sum_{d \geqq 0} S_{d}(\mathfrak{g})$ and so the lemma follows.

Lemma 11. Let $\sigma$ be the representation of $\mathfrak{g}$ on $S(\mathfrak{g})$ as defined above. Then if $F \in S(\mathfrak{g})$ and $X \in \mathfrak{g}$,

$$
\lambda(\sigma(X) F)=[X, \lambda(F)]=X \lambda(F)-\lambda(F) X .
$$

It is clearly sufficient to prove this formula when $F$ is of the form $\bar{Y}_{1} \bar{Y}_{2} \cdots \bar{Y}_{k}, Y_{1}, \cdots, Y_{k}$ being in g. Set $Y_{i}^{\prime}=\left[X, Y_{i}\right](1 \leqq i \leqq k)$; since $\sigma(X)$ is a derivation, $\sigma(X) F$ is the sum of the $\operatorname{l}$ products obtained from $\bar{Y}_{1} \cdots \bar{Y}_{k}$ by replacing successively each one of the factors $\bar{Y}_{i}$ by $\bar{Y}_{i}^{\prime}$. On the other hand the mapping $b \rightarrow[X, b](b \in \mathfrak{B})$ is a derivation of $\mathfrak{B}$. It follows that for any permutation $\omega$ of $(1,2, \cdots, k),\left[X, Y_{\omega(1)} Y_{\omega(2)} \cdots Y_{\omega(k)}\right]$ is the sum of the products obtained from $Y_{\omega(1)} Y_{\omega(2)} \cdots Y_{\omega(k)}$ by replacing successively each one of the factors $Y_{\omega(i)}$ by its transform $\left[X, Y_{\omega(i)}\right]=Y_{\omega(i)}^{\prime}$. The formula $\lambda(\sigma(X) F)=[X, \lambda(F)]$ now follows immediately.

CoRollary. The center of the algebra $\mathfrak{B}$ is the image under $\lambda$ of the set of elements $F$ in $S(\mathfrak{g})$ such that $\sigma(X) F=0$ for all $X \in \mathfrak{g}$.

This is an immediate consequence of Lemma 11. 
If a vector space $V$ (of finite dimension) is the direct sum of two subspaces $V^{\prime}$ and $V^{\prime \prime}$, we may obviously identify $S\left(V^{\prime}\right)$ and $S\left(V^{\prime \prime}\right)$ with subalgebras of $S(V)$ and the bilinear mapping $\left(F^{\prime}, F^{\prime \prime}\right) \rightarrow F^{\prime} F^{\prime \prime}$ defines an isomorphism of the tensor product $S\left(V^{\prime}\right) \times S\left(V^{\prime \prime}\right)$ with $S(V)$ as is easily seen by making use of a base of $V$ which is composed of a base of $V^{\prime}$ and a base of $V^{\prime \prime}$.

Lemma 12. Let $\mathfrak{g}$ be the direct sum of two subspaces $\mathfrak{g}^{\prime}$ and $\mathfrak{g}^{\prime \prime}$. The bilinear mapping $\left(F^{\prime}, F^{\prime \prime}\right) \rightarrow \lambda\left(F^{\prime}\right) \lambda\left(F^{\prime \prime}\right)\left(F^{\prime} \in S\left(\mathfrak{g}^{\prime}\right), F^{\prime \prime} \in S\left(\mathfrak{g}^{\prime \prime}\right)\right)$ defines a linear isomorphism of $S\left(\mathfrak{g}^{\prime}\right) \times S\left(\mathfrak{g}^{\prime \prime}\right)$ with $\mathfrak{B}$. If $d$ is an integer $\geqq 0$, denote by $S_{d}(\mathfrak{g})$, $S_{d}\left(\mathfrak{g}^{\prime}\right)$, and $S_{d}\left(\mathfrak{g}^{\prime \prime}\right)$ the spaces of homogeneous elements of degree $d$ in $S(\mathfrak{g})$, $S\left(\mathfrak{g}^{\prime}\right)$, and $S\left(\mathfrak{g}^{\prime \prime}\right)$ respectively. Then

$$
\sum_{e \leqq d} \lambda\left(S_{e}(\mathfrak{g})\right)=\sum_{d^{\prime \prime}+d^{\prime \prime} \leqq d} \lambda\left(S_{d^{\prime}}\left(\mathfrak{g}^{\prime}\right)\right) \lambda\left(S_{d^{\prime \prime}}\left(\mathfrak{g}^{\prime \prime}\right)\right) .
$$

In this lemma, as in the rest of this paper, we make the following convention: if $A$ and $B$ are linear subspaces of an associative algebra, we denote by $A B$ the vector space spanned by all elements $a b(a \in A, b \in B)$.

Consider a base $\left\{X_{1}, \cdots, X_{n}\right\}$ of $g$ which is composed of a base $\left\{X_{1}, \cdots, X_{n^{\prime}}\right\}$ of $\mathfrak{g}^{\prime}$ and a base $\left\{X_{n^{\prime}+1}, \cdots, X_{n^{\prime}+n^{\prime \prime}}\right\}$ of $\mathfrak{g}^{\prime \prime}\left(n=n^{\prime}+n^{\prime \prime}\right)$. We denote by $M$ the set of all monomials in $\bar{X}_{1}, \cdots, \bar{X}_{n}$, by $M^{\prime}$ the set of monomials in $\bar{X}_{1}, \cdots, \bar{X}_{n^{\prime}}$, by $M^{\prime \prime}$ the set of monomials in $\bar{X}_{n^{\prime}+1}, \cdots$, $\bar{X}_{n^{\prime}+n^{\prime \prime}}$, by $M_{d}$ the set of monomials of degree $d$ in $M$, by $M_{d}^{\prime}$ the set $M^{\prime} \cap M_{d}$, and by $M_{d}^{\prime \prime}$ the set $M^{\prime \prime} \cap M_{d}$. The space $\sum_{e \leqq d} \lambda\left(S_{e}(\mathfrak{g})\right)$ is spanned by the elements $\lambda(\mu), \mu \in \mathrm{U}_{e \leq d} M_{\theta}$. We shall prove by induction on $d$ that it is also spanned by $\lambda\left(\mu^{\prime}\right) \lambda\left(\mu^{\prime \prime}\right)$ where $\mu^{\prime} \in M_{d^{\prime}}^{\prime}, \mu^{\prime \prime} \in M_{d^{\prime \prime}}^{\prime \prime}$, and $d^{\prime}+d^{\prime \prime} \leqq d$. This is obviously true for $d=0$. Assume that it is true for $d$. It is proved in [8(a)] that if $F \in S_{k}(\mathfrak{g}), F^{\prime} \in S_{k^{\prime}}(\mathfrak{g})$ then

$$
\lambda(F) \lambda\left(F^{\prime}\right) \equiv \lambda\left(F F^{\prime}\right) \bmod \sum_{m<k+k^{\prime}} \lambda\left(S_{m}(\mathfrak{g})\right) .
$$

It follows immediately that the condition $d^{\prime}+d^{\prime \prime} \leqq d+1$ implies $\lambda\left(S_{d^{\prime}}\left(\mathfrak{g}^{\prime}\right)\right) \lambda\left(S_{d^{\prime \prime}}\left(\mathfrak{g}^{\prime \prime}\right)\right) \subset \sum_{e \leqq d+1} \lambda\left(S_{e}(\mathfrak{g})\right)$. On the other hand every $\mu \in M_{d+1}$ may be written in the form $\mu^{\prime} \mu^{\prime \prime}$ where $\mu^{\prime} \in M_{d^{\prime}}^{\prime}, \mu^{\prime \prime} \in M_{d^{\prime \prime}}^{\prime \prime}$, and $d^{\prime}+d^{\prime \prime}=d+1$. Hence it follows from the above formula and our inductive assumption that $\lambda(\mu) \in \sum_{d^{\prime \prime}+d^{\prime \prime} \leqq d+1} \lambda\left(S_{d^{\prime}}\left(\mathfrak{g}^{\prime}\right)\right) \lambda\left(S_{d^{\prime \prime}}\left(\mathfrak{g}^{\prime \prime}\right)\right)$ and this proves our assertion for $d+1$. Since every element $\mu \in M$ can be written in exactly one way in the form $\mu^{\prime} \mu^{\prime \prime}\left(\mu^{\prime} \in M^{\prime}, \mu^{\prime \prime} \in M^{\prime \prime}\right)$, we see that for every $d$ the elements $\lambda\left(\mu^{\prime}\right) \lambda\left(\mu^{\prime \prime}\right)$ $\left(\mu^{\prime} \in M_{d^{\prime}}^{\prime}, \mu^{\prime \prime} \in M_{d^{\prime \prime}}^{\prime \prime}, d^{\prime}+d^{\prime \prime} \leqq d\right)$ form a base of $\sum_{\bullet} \leqq d \lambda\left(S_{e}(\mathfrak{g})\right)$. This being true for every $d$, the lemma follows.

It is clear that $\lambda(S(\mathfrak{l}))=\mathfrak{X}$. We shall denote by $\mathfrak{B}$ the set $\lambda(S(\mathfrak{p}))$ and by $\mathfrak{B}_{d}$ the set $\lambda\left(S_{d}(\mathfrak{p})\right)$.

LEMMA 13. We have $\lambda\left(S_{d}(\mathfrak{p}) S(\mathfrak{l})\right) \subset \sum_{\varrho \leqq d} \mathfrak{B}_{d} \mathfrak{X}$ and $\lambda\left(S_{d}(\mathfrak{p}) S(\mathfrak{l})\right) \lambda\left(S_{d^{\prime}}(\mathfrak{p}) S(\mathfrak{l})\right)$ $\subset \sum_{\odot \leqq d+d^{\prime}} \mathfrak{B}_{\odot} \mathfrak{X}$. 
The first formula follows immediately from Lemma 12 . In order to prove the second formula, it will be sufficient to show that

$$
\left(\mathfrak{P}_{d} \mathfrak{X}\right)\left(\mathfrak{P}_{d^{\prime}} \mathfrak{X}\right) \subset \sum_{e \leqq d+d^{\prime}} \mathfrak{B}_{e} \mathfrak{X} .
$$

Let $\mathfrak{p}^{d}$ denote the space spanned by the products of $d$ elements in $\mathfrak{p}$. First we prove that $\mathfrak{X p}^{d} \subset \mathfrak{p}^{d} \mathfrak{X}$. This is true for $d=0$. Let $\mathfrak{X}^{\prime}$ be the set of all $u \in \mathfrak{B}$ such that $u \mathfrak{p} \subset \mathfrak{p} \mathfrak{X}$. Then $\mathfrak{X}^{\prime}$ is a subalgebra of $\mathfrak{B}$ and $1 \in \mathfrak{X}^{\prime}$. Moreover if $X \in \mathfrak{l}$ and $Y \in \mathfrak{p}$, then $X Y=Y X+[X, Y] \in \mathfrak{p} \mathfrak{X}$ since $[X, Y] \in \mathfrak{p}$. Hence $\mathfrak{t}$ and therefore $\mathfrak{X}$ is contained in $\mathfrak{X}^{\prime}$ which shows that $\mathfrak{X} \subset \subset \mathfrak{p}$. Now assume that our assumption is true for some $d$. Then $\mathfrak{X p}^{d+1}=\left(\mathfrak{X p}^{d}\right) \mathfrak{p} \subset \mathfrak{p}^{d} \mathfrak{X} \mathfrak{p} \subset \mathfrak{p}^{d+1} \mathfrak{X}$. This proves it for $d+1$. But it is clear that $\mathfrak{B}_{d} \subset \mathfrak{p}^{d}$ and therefore

$$
\left(\mathfrak{P}_{d} \mathfrak{X}\right)\left(\mathfrak{P}_{d^{\prime}} \mathfrak{X}\right) \subset \mathfrak{p}^{d} \mathfrak{X} \mathfrak{p}^{d^{\prime}} \mathfrak{X} \subset \mathfrak{p}^{d+d^{\prime}} \mathfrak{X} .
$$

Hence it would be sufficient to prove that $\mathfrak{p}^{d} \mathfrak{X} \subset \sum_{e \leqq d} \mathfrak{P}_{e} \mathfrak{X}$. This is true for $d=0,1$. Assume that it is true for $d$. Then

$$
\mathfrak{p}^{d+1} \mathfrak{X} \subset \sum_{e \leqq d} \mathfrak{p} \mathfrak{B}_{e} \mathfrak{X} \text {. }
$$

But $\mathfrak{p M}_{e} \subset \lambda\left(S_{1}(\mathfrak{g})\right) \lambda\left(S_{e}(\mathfrak{g})\right)$ and therefore from formula (1) above $\mathfrak{p \mathfrak { B } _ { e }}$ $\subset \sum_{e^{\prime} \leqq e+1} \lambda\left(S_{e^{\prime}}(\mathfrak{g})\right)$ and this is contained in $\sum_{e^{\prime} \leqq e+1} \mathfrak{B}_{e^{\prime}} \mathfrak{X}$ in virtue of Lemma 12. Since this proves our formula for $d+1$ the lemma follows.

Let $\mathrm{g}^{*}$ be the space dual to $g$, i.e., the space of linear functions on $\mathfrak{g}$. Then the fundamental bilinear form $B(X, Y)$ of $\mathfrak{g}$ defines an isomorphism $I$ of $\mathfrak{g}$ with $\mathfrak{g}^{*}$, which assigns to every $X \in \mathfrak{g}$ the linear function $Y \rightarrow B(X, Y)$ on g. (This is an isomorphism since $B$ is nondegenerate.) $I$ may be extended uniquely to an isomorphism of $S(\mathfrak{g})$ with the symmetric algebra $S\left(\mathfrak{g}^{*}\right)$ on $\mathfrak{g}^{*}$. We denote this extension again by $I$. Let $\left\{x_{1}^{*}, \cdots, x_{n}^{*}\right\}$ be a base for $\mathfrak{g}^{*}$ and $F$ any element in $S\left(\mathfrak{g}^{*}\right)$. Then $F=P\left(x_{1}^{*}, \cdots, x_{n}^{*}\right)$ where $P$ is a polynomial. Since $x_{1}^{*}, \cdots, x_{n}{ }^{*}$ are linear functions on $\mathfrak{g}, P\left(x_{1}^{*}, \cdots, x_{n}^{*}\right)$ represents a function on $\mathfrak{g}$, namely the function $F^{\prime}: X \rightarrow P\left(x_{1}^{*}(X), \cdots, x_{n}^{*}(X)\right)$ $(X \in \mathfrak{g})$. It is clear that the mapping $F \rightarrow F^{\prime}$ is isomorphic and does not depend on the choice of the base $\left\{x_{1}^{*}, \cdots, x_{n}^{*}\right\}$. The functions $F^{\prime}$ are called the polynomial functions on $\mathfrak{g}$. Henceforth we shall identify $S(\mathfrak{g})$ with $S\left(\mathfrak{g}^{*}\right)$ and the algebra of polynomial functions on $\mathrm{g}$ under the mappings $F \rightarrow I(F)$ $\rightarrow(I(F))^{\prime}(F \in S(\mathfrak{g}))$. Since $B(X, Y)=0, X \in \mathfrak{f}$, and $Y \in \mathfrak{p}, \mathfrak{p}$ is identified with the space of linear functions on $\mathfrak{g}$ which are zero on $\mathfrak{l}$. We may therefore identify $S(\mathfrak{p})$ with the algebra of polynomial functions on the subspace $\mathfrak{p}$ of $\mathfrak{g}$. Let $\left\{x_{1}, \cdots, x_{p}, x_{p+1}, \cdots, x_{n}\right\}$ be a base for $g$ such that $\left\{x_{1}, \cdots, x_{p}\right\}$ and $\left\{x_{p+1}, \cdots, x_{n}\right\}$ are bases for $\mathfrak{p}$ and $\mathfrak{t}$ respectively. Then if $P$ is a polynomial in $n$ variables, the restriction of the polynomial function $F=P\left(x_{1}, \cdots, x_{n}\right)$ on $\mathfrak{p}$ is $P\left(x_{1}, \cdots, x_{p}, 0, \cdots, 0\right)$.

3. Proof of Theorem 1. Let $\mathfrak{A}$ be an associative algebra with a unit element 1 and $\mathfrak{M}$ a left ideal in $\mathfrak{A}$. Then the space $\mathfrak{A}^{*}=\mathfrak{A} / \mathfrak{M}$ is an $\mathfrak{A}$-module and if 
we assign to every $x \in \mathfrak{A}$ the mapping $\pi(x): a^{*} \rightarrow x a^{*}$ of $\mathfrak{A}^{*}$ into itself we obtain a representation $\pi$ of $\mathfrak{A}$, which we call the natural representation of $\mathfrak{A}$ on $\mathfrak{A} / \mathfrak{M}$. Let $\mathfrak{l}$ be a finite-dimensional subspace of $\mathfrak{A}$ which is a Lie algebra under the bracket operation $[X, Y]=X Y-Y X(X, Y \in \mathfrak{l})$. Then $\pi$ defines a representation of this Lie algebra which will also be called the natural representation of $\mathfrak{l}$ on $\mathfrak{A} / \mathfrak{M}$.

Theorem 1. Let $\mathfrak{Y}$ be a left ideal in $\mathfrak{X}$. Assume that $\mathfrak{X} / \mathfrak{Y}$ is finite-dimensional and the natural representation of $\mathfrak{t}$ on $\mathfrak{X} / \mathfrak{Y}$ is semisimple. Then the natural representation of $\mathfrak{t}$ on $\mathfrak{B} / \mathfrak{B Y}$ is quasi-semisimple. Let $\mathfrak{D}$ be any equivalence class of finite-dimensional simple representations of $\mathfrak{t}$ and let $\mathfrak{B}_{\mathfrak{D}}^{*}$ be the set of all elements of $\mathfrak{B}^{*}=\mathfrak{B} / \mathfrak{B V}$ which transform according to $\mathfrak{D}$ under the natural representation $\pi$ of $\mathfrak{t}$ on $\mathfrak{B}^{*}$. Then $\mathfrak{B}_{\mathfrak{D}}^{*}$ is a finite module over the center $\mathfrak{B}$ of $\mathfrak{B}$.

In order to clarify the last assertion we first observe that $\mathfrak{B}_{\mathfrak{D}}^{*}$ is actually a module over 3 . Let $x$ be an element of $\mathfrak{B}_{\mathfrak{D}}^{*}$ and let $W$ be the smallest $\pi$-stable subspace of $\mathfrak{B}^{*}$ containing $x$. Let $\pi_{W}(X)(X \in \mathfrak{l})$ denote the restriction of $\pi(X)$ on $W$. Then $\pi_{W}$ is semisimple and every simple component of $\pi_{W}$ is contained in $\mathfrak{D}$. Let $z \in \mathfrak{Z}$; then the mapping $y \rightarrow z y(y \in W)$ maps $W$ upon a space $z W$. If $X \in \mathfrak{l}, X z y=z X y \in z W$; thus $z W$ is $\pi$-stable and $\pi(X) z y$ $=z(\pi(X) y)$. It follows immediately (see Chevalley [4, Chap. VI]) that the representation of $\mathfrak{t}$ induced on $z W$ is semisimple and all its simple components are in $\mathfrak{D}$. Hence $z x \in \mathfrak{B}_{\mathfrak{D}}^{*}$ and this shows that $\mathfrak{B}_{\mathfrak{D}}^{*}$ is a $\mathfrak{B}$-module.

The proof of Theorem 1 will be divided into several parts.

1. Proof of the first assertion. We have defined above the canonical mapping $\lambda$ of $S(\mathfrak{g})$ onto $\mathfrak{B}$. We consider now the tensor product $S(\mathfrak{p}) \times(\mathfrak{X} / \mathfrak{Y})$. Since $\mathfrak{Y} \subset \mathfrak{B} \mathfrak{Y}$, there is a natural mapping $f \rightarrow f^{*}(f \in \mathfrak{X} / \mathfrak{V})$ of $\mathfrak{X} / \mathfrak{V}$ into $\mathfrak{B}^{*}$ $=\mathfrak{B} / \mathfrak{B Y}$. Define a linear mapping $\Gamma$ of $S(\mathfrak{p}) \times(\mathfrak{X} / \mathfrak{Y})$ into $\mathfrak{B}^{*}$ as follows:

$$
\Gamma(F \times f)=\lambda(F) f^{*} \quad(F \in S(\mathfrak{p}), f \in \mathfrak{X} / \mathfrak{Y}) .
$$

(We recall that $\mathfrak{B}^{*}$ is a $\mathfrak{B}$-module and so $\lambda(F)$ operates on $\mathfrak{B}^{*}$.) We shall prove that $\Gamma$ is a linear isomorphism of $S(\mathfrak{p}) \times(\mathfrak{X} / \mathfrak{Y})$ with $\mathfrak{B}^{*}$. Select a base $\left(s_{i}\right)_{i \in I}$ for $S(\mathfrak{p})$ and a base $\left(t_{j}\right)_{j \in J}$ for $S(\mathfrak{t})$. We may suppose that one of the elements $s_{i}$, say $s_{0}$, is equal to 1 . The mapping $\lambda$ induces an isomorphism of $S(\mathfrak{l})$ with $\mathfrak{X}$. Hence we may assume that the base $\left(t_{j}\right)_{j \in J}$ is so chosen that for a suitable subset $J^{\prime}$ of $J$ the elements $\left(\lambda\left(t_{j}\right)\right)_{j \in J^{\prime}}$ form a base for $\mathfrak{Y}$. From Lemma 12 the elements $\lambda\left(s_{i}\right) \lambda\left(t_{j}\right)(i \in I, j \in J)$ form a base for $\mathscr{B}$ and, if $j \in J^{\prime}$, $\lambda\left(s_{i}\right) \lambda\left(t_{j}\right) \in \mathfrak{B Y}$. Conversely every element of $\mathfrak{B Y}$ is in the space spanned by the elements $\lambda\left(s_{i}\right) \lambda\left(t_{j}\right) \lambda\left(t_{j^{\prime}}\right) \quad\left(i \in I, j \in J, j^{\prime} \in J^{\prime}\right)$. But since $\mathfrak{Y}$ is a left ideal in $\mathfrak{X}, \lambda\left(t_{j}\right) \lambda\left(t_{j^{\prime}}\right) \in \mathfrak{Y}$ and so $\lambda\left(t_{j}\right) \lambda\left(t_{j^{\prime}}\right)$ lies in the space spanned by $\lambda\left(t_{j^{\prime \prime}}\right)\left(j^{\prime \prime} \in J^{\prime}\right)$. This proves that the elements $\lambda\left(s_{i}\right) \lambda\left(t_{j^{\prime}}\right)\left(i \in I, j^{\prime} \in J^{\prime}\right)$ form a base for $\mathfrak{B V}$. Since $\mathfrak{X}$ is spanned by the elements $\lambda\left(s_{0}\right) \lambda\left(t_{j}\right)(j \in J)$, it follows that $\mathfrak{B Y} \cap \mathfrak{X}$ $=\mathfrak{Y}$. We may therefore identify $\mathfrak{X} / \mathfrak{Y}$ with its image in $\mathfrak{B}^{*}$. Let $J^{\prime \prime}$ be the complement of $J^{\prime}$ in $J$. Let $\lambda^{*}\left(t_{j}\right)\left(j \in J^{\prime \prime}\right)$ denote the residue class of $\lambda\left(t_{j}\right)$ modulo 
V (or $\mathfrak{B Z})$. Then the elements $\lambda\left(s_{i}\right) \lambda^{*}\left(t_{j}\right)\left(i \in I, j \in J^{\prime \prime}\right)$ form a base for $\mathfrak{B}^{*}$. On the other hand the elements $\lambda^{*}\left(t_{j}\right)\left(j \in J^{\prime \prime}\right)$ form a base of $\mathfrak{X} / \mathfrak{Y}$ and $\Gamma\left(s_{i} \times \lambda^{*}\left(t_{j}\right)\right)=\lambda\left(s_{i}\right) \lambda^{*}\left(t_{j}\right) \quad\left(i \in I, j \in J^{\prime \prime}\right)$. This proves that $\Gamma$ is a linear isomorphism of $S(\mathfrak{p}) \times(\mathfrak{X} / \mathfrak{V})$ with $\mathfrak{B}^{*}$.

For any $X \in \mathfrak{l}$ let $\sigma(X)$ denote the derivation of $S(\mathfrak{g})$ which coincides with ad $X$ on $\mathfrak{g}$. Then we have seen in Lemma 10 that $\sigma$ is a quasi-semisimple representation of $\mathfrak{t}$. On the other hand ad $X$ maps $\mathfrak{p}$ into itself and therefore $\sigma(X)$ maps $S(\mathfrak{p})$ into itself. Let $\rho(X)$ denote the restriction of $\sigma(X)$ on $S(\mathfrak{p})$. The representation $\rho$ is quasi-semisimple (Lemma 6 ). Let $\mu$ be the natural representation of $\mathfrak{t}$ on $\mathfrak{X} / \mathfrak{V}$. Then $\mu$ is of finite degree and semisimple. From Lemma 8 , the representation $\rho+\mu$ on $S(\mathfrak{p}) \times(\mathfrak{X} / \mathfrak{V})$ is quasi-semisimple. The first assertion of Theorem 1 will therefore be proved if we show that $\pi$ is equivalent to $\rho+\mu$. Now if $F \in S(\mathfrak{p}), f \in \mathfrak{X} / \mathfrak{V}$, and $X \in \mathfrak{l}$,

$$
((\rho+\mu)(X))(F \times f)=\rho(X) F \times f+F \times X f .
$$

But from Lemma 11,

$$
\lambda(\rho(X) F)=[X, \lambda(F)]
$$

Hence

$$
\begin{aligned}
\Gamma(\rho(X) F \times f)+\Gamma(F \times X f) & =([X, \lambda(F)]) f+\lambda(F) X f \\
& =X \lambda(F) f=\pi(X)(\Gamma(F \times f)) .
\end{aligned}
$$

Since $\Gamma$ is an isomorphism, $\pi$ is equivalent to $\rho+\mu$.

2.1. Proof of the second assertion. First reduction. Let $\nu$ be a simple representation of $\mathfrak{t}$ which is contragredient to the representations of the class $\mathfrak{D}$ and let $V$ be the space of the representation $\nu$. Consider the representation $\pi+\nu$ of $\mathfrak{f}$ on the tensor product $\mathfrak{B}^{*} \times V$. An element $\phi \in \mathfrak{B}^{*} \times V$ will be called an invariant if $((\pi+\nu)(X)) \phi=0$ for all $X \in \mathfrak{l}$. Let $\Im$ be the set of these invariants. Suppose $\left\{v_{1}, \cdots, v_{m}\right\}$ is a base for $V$ and $\phi=\sum_{i=1}^{m} b_{i}^{*} X_{v_{i}}\left(b_{i}{ }^{*} \in \mathfrak{B}^{*}\right)$ is in $\Im$. Then the elements $b_{i}^{*}$ belong to $\mathfrak{B}_{\mathfrak{D}}^{*}$. For, if $X \in \mathfrak{l}, \sum_{i=1}^{m} \pi(X) b_{i}^{*} \times v_{i}$ $=-\sum_{i=1}^{m} b_{i}^{*} \times \nu(X) v_{i}$ from which it follows immediately that the elements $\pi(X) b_{i}^{*}$ lie in the space $V^{*}$ spanned by $b_{1}^{*}, \cdots, b_{m}^{*}$ and therefore $V^{*}$ is $\pi$-stable. Let $\pi_{V^{*}}(X)$ denote the restriction of $\pi(X)$ on $V^{*}$. Now $\phi \in V^{*} \times V$ and $\left(\left(\pi_{V^{*}}+\nu\right)(X)\right) \phi=0$ for all $X \in \mathfrak{l}$. If $\phi \neq 0$ this means that $\pi_{V^{*}}$ contains a simple representation contragredient to $\nu$. Since $\operatorname{dim} V^{*} \leqq m$, it follows that $\pi_{V^{*}}$ itself is contragredient to $\nu$ and so lies in $\mathfrak{D}$. Therefore $b_{i}^{*} \in \mathfrak{B}_{\mathfrak{D}}^{*}(1 \leqq i \leqq m)$. On the other hand if $\phi=0, b_{i}^{*}=0(1 \leqq i \leqq m)$ and so our assertion is true trivially. Conversely let $U$ be a $\pi$-stable subspace of $\mathfrak{B}^{*}$ such that the representation $\pi_{U}$ induced on $U$ lies in $\mathfrak{D}$. Then $\pi_{U}$ is contragredient to $\nu$ and so there exists a base $\left\{b_{1}^{*}, \cdots, b_{m}^{*}\right\}$ for $U$ such that $\sum_{i=1}^{m} b_{i}^{*} \times v_{i}$ is an invariant of $\pi+\nu$.

Since $\mathfrak{B}^{*}$ is a $\mathfrak{B}$-module we may also regard $\mathfrak{B}^{*} \times V$ as a $\mathfrak{B}$-module as follows: 


$$
a\left(b^{*} \times v\right)=\left(a b^{*}\right) \times v \quad\left(a \in \mathfrak{B}, b^{*} \in \mathfrak{B}^{*}, v \in V\right) .
$$

If $z \in \mathfrak{Z}$, the operator $\boldsymbol{z}$ on $\mathfrak{B}^{*} \times V$ commutes with $(\pi+\nu)(X)(X \in \mathfrak{l})$. Hence it follows immediately that $\Im$ is a $\mathbf{Z}$-module. If this $\mathbf{Z}$-module is finite, then $\mathfrak{B}_{\mathfrak{D}}^{*}$ is a finite $\mathbb{3}$-module. For let $\phi_{k}(1 \leqq k \leqq q)$ be a finite set of elements in $\Im$ such that $\Im=\sum_{k=1}^{q} 3 \phi_{k}$. Let $\phi_{k}=\sum_{i=1}^{m} b_{k t}^{*} \times v_{i}\left(b_{k t}^{*} \in \mathfrak{B}^{*}\right)$. Then it follows immediately from what we said above that $\mathfrak{B}_{\mathfrak{D}}^{*}=\sum_{k=1}^{q} \sum_{i=1}^{m} B b_{k t}^{*}$.

We have defined above an isomorphism $\Gamma$ of $S(\mathfrak{p}) \times(\mathfrak{X} / \mathfrak{Y})$ with $\mathfrak{B}^{*}$. Now consider the linear isomorphism

$$
F \times f \times v \rightarrow \Gamma(F \times f) \times v \quad(F \in S(\mathfrak{p}), f \in \mathfrak{X} / \mathfrak{V}, v \in V)
$$

of $S(\mathfrak{p}) \times(\mathfrak{X} / \mathfrak{Y}) \times V$ with $\mathfrak{B}^{*} \times V$. This we shall denote again by $\Gamma$. Put $W=(\mathfrak{X} / \mathfrak{Y}) \times V$ and $\xi=\rho+\mu+\nu, \mu^{\prime}=\mu+\nu, \pi^{\prime}=\pi+\nu$. Then if $X \in \mathfrak{l}$ and $A$ $\in S(\mathfrak{p}) \times W, \Gamma(\xi(X) A)=\pi^{\prime}(X) \Gamma(A)$. Let $\mathfrak{\Im}^{\prime}$ be the set of elements $A \in S(\mathfrak{p})$ $\times W$ such that $\xi(X) A=0$ for all $X \in \mathfrak{l}$. Thus $\Gamma$ induces an isomorphism of $\Im^{\prime}$ with $\Im$.

We now regard $S(\mathfrak{p}) \times W$ as a module over $S(\mathfrak{p})$ by the rule $F_{1}\left(F_{2} \times w\right)$ $=\left(F_{1} F_{2}\right) \times w\left(F_{1}, F_{2} \in S(\mathfrak{p}), w \in W\right)$. If $X \in \mathfrak{l}, F \in S(\mathfrak{p}), A \in S(\mathfrak{p}) \times W$, we have $\xi(X) F A=(\rho(X) F) A+F(\xi(X) A)$. Let $\Omega$ denote the set of elements $F \in S(\mathfrak{p})$ such that $\rho(X) F=0$ for all $X \in \mathfrak{l}$. Then $\Im^{\prime}$ is clearly an $\Omega$-module.

On the other hand for any $Z \in g$ the operation ad $Z$ may be extended to a derivation $\tau(Z)$ of the algebra $S(\mathfrak{g})$. If $Z \in \mathfrak{f}$, then the operation $\sigma(Z)$ considered above is identical with $\tau(Z)$. We shall denote by $J$ the set of elements $J \in S(\mathrm{~g})$ such that $\tau(Z) J=0$ for all $Z \in \mathrm{g}$; this is a subalgebra of $S(\mathrm{~g})$. We now regard the elements of $S(\mathfrak{g})$ as polynomial functions on $\mathfrak{g}$ in the manner explained above and denote by $\mathfrak{J}_{\mathfrak{p}}$ the set of restrictions to $\mathfrak{p}$ of all functions $J$ in $\jmath_{\text {. Then }} \mathfrak{J}_{\mathfrak{p}} \subset \Omega$. For, let $J \in J^{\prime}$ and let $J^{\prime}$ be its restriction to $\mathfrak{p}$. Then $J^{\prime}$ is the unique element of $S(\mathfrak{p})$ such that $J-J^{\prime}$ belongs to the ideal $\Re$ generated by $\mathfrak{l}$ in $S(\mathfrak{g})$. Let $X$ be an element of $\mathfrak{t}$. Then $\sigma(X) J=0$. On the other hand since ad $X$ maps the spaces $\mathfrak{l}$ and $\mathfrak{p}$ into themselves, $\sigma(X)$ maps $\Omega$ and $S(\mathfrak{p})$ into themselves and so $\rho(X) J^{\prime}=\tau(X) J^{\prime} \in \Omega \cap S(\mathfrak{p})=\{0\}$. Hence $J^{\prime} \in \Omega$. It is clear that $J_{\mathfrak{p}}$ is a algebra of $\Omega$. We shall see that in order to prove that $\Im$ is a finite $\mathbf{3}$-module it is sufficient to show that $\mathfrak{S}^{\prime}$ is a finite $\mathfrak{J}_{\mathfrak{p}}$-module.

Assume that $\mathfrak{S}^{\prime}$ is a finite $\mathfrak{J}_{\mathfrak{p}}$-module. For any integer $d \geqq 0$ let $S_{d}(\mathfrak{p})$ denote the space of homogeneous elements of degree $d$ of $S(\mathfrak{p})$. Then $S_{d}(\mathfrak{p}) \times W$ is mapped into itself by the operations of $\xi(\mathfrak{l})$. Therefore we can find a finite number of elements $A_{i} \in \mathcal{Y}^{\prime}(1 \leqq i \leqq r)$ each of which lies in some $S_{d}(\mathfrak{p}) \times W$, say $S_{d_{i}}(\mathfrak{p}) \times W$, such that $\Im^{\prime}=\sum_{i=1}^{r} J_{p} A_{i}$. The space $\Im_{\infty}=\sum_{i=1}^{r} 3 \Gamma\left(A_{i}\right)$ is contained in $\Im$. We now intend to prove that $\Im_{\infty}=\Im$. Put

$$
\Im_{d}^{\prime}=\Im^{\prime} \cap\left(\sum_{0 \leqq e \leqq d} S_{e}(\mathfrak{p}) \times W\right), \Im_{d}=\Gamma\left(\Im_{d}^{\prime}\right) .
$$

The set of those $A_{i}$ for which $d_{i}=0$ obviously generates the space $\mathfrak{I}^{\prime} \cap W$ 
( $W$ being identified in the usual manner to the subspace $1 \times W$ of $S(\mathfrak{p}) \times W$ ). It follows that $\Im_{0} \subset \Im_{\infty}$. It will therefore be sufficient to prove that for every $d \geqq 0, \Im_{d+1} \subset \Im_{d}+\Im_{\infty}$.

Let $A \in \mathcal{S}^{\prime} \cap\left(S_{d+1}(\mathfrak{p}) \times W\right)$ and let $A=\sum_{i=1}^{r} F_{i} A_{i}$ with $F \in J_{\mathfrak{p}}$. It is clear that the homogeneous components of any element of $J_{p}$ also belong to $J_{p}$. We may therefore assume that $F_{i} \in S_{d+1-d_{i}}(\mathfrak{p})\left(S_{e}(\mathfrak{p})=\{0\}\right.$ if $\left.e<0\right)$. Let $\left\{w_{1}, \cdots, w_{a}\right\}$ be a base for $W$ and let $A_{i}=\sum_{j=1}^{a} A_{i j} \times w_{j}$ with $A_{i j} \in S_{d_{i}}(p)$. Then $\Gamma(A)=\sum_{i=1}^{r} \sum_{j=1}^{a} \lambda\left(F_{i} A_{i j}\right) w_{j}$. Each $F_{i}$ is the restriction to $\mathfrak{p}$ of an element $J_{i}$ of $J$ and we may assume that $J_{i}$ is homogeneous of degree $d+1-d_{i}$ which is the degree of $F_{i}$. It follows that $F_{i}-J_{i} \in \sum_{e<d+1-d_{i}} S_{e}(\mathfrak{p}) S(\mathfrak{l})$. Hence

$$
F_{i} A_{i j}-J_{i} A_{i j} \in \sum_{e \leqq d} S_{\ell}(\mathfrak{p}) S(\mathfrak{l}) .
$$

Therefore from Lemma 13,

$$
\Gamma(A)-\sum_{i=1}^{r} \sum_{j=1}^{a} \lambda\left(J_{i} A_{i j}\right) w_{j} \in \sum_{e \leq d}\left(\mathfrak{P}_{e} \mathfrak{X}\right) W
$$

where $\mathfrak{B}_{e}=\lambda\left(S_{e}(\mathfrak{p})\right)$. On the other hand we know (see $\left.[8(\mathrm{a})]\right)$ that

$$
\lambda\left(J_{i} A_{i j}\right) \equiv \lambda\left(J_{i}\right) \lambda\left(A_{i j}\right) \bmod \sum_{\mathfrak{e} \leqq d} \lambda\left(S_{e}(\mathfrak{g})\right)
$$

where $S_{e}(\mathfrak{g})$ is the space of homogeneous elements of degree $e$ in $S(\mathfrak{g})$. Since $\lambda\left(S_{e}(\mathfrak{g})\right) \subset \sum_{e^{\prime} \leqq e} \mathfrak{B}_{e^{\prime}} \mathfrak{X}$ from Lemma 12, it follows that

$$
\Gamma(A)-\sum_{i=1}^{r} \sum_{j=1}^{a} \lambda\left(J_{i}\right) \lambda\left(A_{i j}\right) w_{j} \in \sum_{e \leqq d}\left(\mathfrak{B}_{\mathfrak{e}} \mathfrak{X}\right) W .
$$

Since $W$ is the subspace $((\mathfrak{X}+\mathfrak{B Z}) / \mathfrak{B} \mathfrak{Y}) \times V$ of $\mathfrak{B}^{*} \times V$, it is clear that $\mathfrak{X} W \subset W$ and therefore

$$
\sum_{e \leqq d}\left(\mathfrak{P}_{e} \mathfrak{X}\right) W \subset \Gamma\left(\left(\sum_{e \leqq d} S_{e}(\mathfrak{p})\right) \times W\right) .
$$

On the other hand

$$
\sum_{i=1}^{r} \sum_{j=1}^{a} \lambda\left(J_{i}\right) \lambda\left(A_{i j}\right) w_{j}=\sum_{i=1}^{r} \lambda\left(J_{i}\right) \Gamma\left(A_{i}\right) .
$$

The elements $\lambda\left(J_{i}\right)$ are in 8 in virtue of the corollary to Lemma 11. Hence $\sum_{i=1}^{r} \lambda\left(J_{i}\right) \Gamma\left(A_{i}\right) \in \Im_{\infty}$ and therefore

$$
\Gamma(A)-\sum_{i=1}^{r} \lambda\left(J_{i}\right) \Gamma\left(A_{i}\right) \in \Im \cap \Gamma\left(\sum_{e \leqq d} S_{e}(\mathfrak{p}) \times W\right)=\Im_{d} .
$$

This proves that $\Gamma(A) \in \Im_{d}+\Im_{\infty}$ and therefore $\Im_{d+1} \subset \Im_{d}+\Im_{\infty}$. Hence $\Im=\Im_{\infty}$. 
2.2. Second reduction. We shall now establish that the ring $\Omega$ introduced above is a finite module over its subring $J_{\mathfrak{p}}$. This will reduce the problem of proving that $\mathfrak{\Im}^{\prime}$ is a finite $\mathfrak{J}_{\mathfrak{p}}$-module to that of proving that it is finite $\Omega$-module.

Let $\mathfrak{h}$ be the Cartan subalgebra of $\mathfrak{g}$ as defined in Lemma 4 . Let $p=\operatorname{dim} \mathfrak{p}$ and $h=\operatorname{dim}(\mathfrak{h} \cap \mathfrak{p})$. We shall first show that $\Omega$ cannot contain more than $h$ algebraically independent elements. Let $\left(Y_{1}, \ldots, Y_{p}\right)$ be a base of $\mathfrak{p}$. We have identified every element $Y$ of $\mathfrak{g}$ to the linear function $Z \rightarrow B(Y, Z)$ on $\mathfrak{g}$. Let $y_{1}, \cdots, y_{p}$ be the elements $Y_{1}, \cdots, Y_{p}$ regarded as linear functions on g. These functions are zero on $t$. An element of $\Omega$ may be written as a polynomial $F\left(y_{1}, \cdots, y_{p}\right)$ in $y_{1}, \cdots, y_{p}$ and $\sum_{i=1}^{p} \partial F\left(y_{1}, \cdots, y_{p}\right) / \partial y_{i}\left(\rho(X) y_{i}\right)$ $=0$ for all $X \in \mathfrak{l}$. The function $\rho(X) y_{i}$ is the function $Z \rightarrow B\left(\left[X, Y_{i}\right], Z\right)$ $=-B\left(Y_{i},[X, Z]\right)$ on $\mathfrak{g}$. Let $Z$ be any element of $\mathfrak{p}$. Put $\zeta_{i}=y_{i}(Z)(1 \leqq i \leqq p)$. Then

$$
\sum_{i=1}^{p} \frac{\partial F}{\partial y_{i}}\left(\zeta \mathrm{i}, \cdots, \zeta_{p}\right) y_{i}([X, Z])=0 .
$$

Let $\left\{X_{1}, \cdots, X_{k}\right\}$ be a base for 1 . Then the rank $r(Z)$ of the matrix $\left(y_{i}\left(\left[X_{j}, Z\right]\right)\right)_{1 \leqq i \leqq p, 1 \leqq j \leqq k}$ is the dimension of the space spanned by the elements $[X, Z]$ for all $X \in \mathfrak{l}$. Let $r=\max _{Z \in \mathfrak{p}} r(Z)$. Then there exists a polynomial function $G \neq 0$ on $\mathfrak{p}$ such that $r(Z)=r$ if $G(Z) \neq 0(Z \in \mathfrak{p})$. It follows from the above relation that if $F_{1}, \cdots, F_{q}$ are in $\Omega$, the rank of the Jacobian matrix $\left(\partial F_{i} / \partial y_{j}\right)_{1 \leqq i \leqq q, 1 \leqq j \leqq p}$ for $y_{i}=\zeta_{i}$ is $\leqq p-r(Z)$. Hence there cannot be more than $p-r$ algebraically independent elements among $F_{1}, \cdots, F_{q}$. Now it is clear that $r(Z)$ is equal to the difference between the dimension of $\mathfrak{t}$ and that of the space of elements $X \in \mathfrak{l}$ which commute with $Z$. Making use of Lemma 4, we see that there exists an element $Z \in \mathfrak{p}$ such that $r(Z)=p-h$. Hence $r \geqq p-h$ and therefore there cannot be more than $h$ algebraically independent elements among $F_{1}, \cdots, F_{q}$. This proves our assertion.

The bilinear form $B$ is nondegenerate on $\mathfrak{h}$. Since $\mathfrak{h}=\mathfrak{h}_{\mathfrak{p}}+\mathfrak{h}_{\mathfrak{t}}$ and $\mathfrak{t}$ and $\mathfrak{p}$ are mutually orthogonal under $B$, it follows that $B$ is nondegenerate on $\mathfrak{h}_{\mathfrak{p}}$. We may therefore assume that the base $\left\{Y_{1}, \cdots, Y_{p}\right\}$ for $\mathfrak{p}$ is so chosen that $Y_{1}, \cdots, Y_{h}$ form a base for $\mathfrak{h}_{\mathfrak{p}}=\mathfrak{h} \cap \mathfrak{p}$ and $B\left(Y_{i}, Y_{j}\right)=0$ if $i \leqq h<j$. The restrictions of $y_{1}, \cdots, y_{h}$ to $\mathfrak{h}_{\mathfrak{p}}$ are then linearly independent and the ring generated by $y_{1}, \cdots, y_{h}$ may be identified with the algebra $S\left(\mathfrak{h}_{\mathfrak{p}}\right)$ of polynomial functions on $\mathfrak{h}_{\mathfrak{p}}$ (i.e., those functions on $\mathfrak{h}_{\mathfrak{p}}$ which may be written as polynomials in linear functions). The restriction of an element $F\left(y_{1}, \cdots, y_{p}\right)$ of $S(\mathfrak{p})$ to $\mathfrak{h}_{\mathfrak{p}}$ is $F\left(y_{1}, \cdots, y_{h}, 0, \cdots, 0\right)$. Let $\mathfrak{h}_{\mathfrak{p}}$ be the algebra of restrictions to $\mathfrak{h}_{\mathfrak{p}}$ of elements of $\mathfrak{J}_{\mathfrak{p}}$. We shall prove $\mathfrak{J}_{\mathfrak{p}}$ contains $h$ algebraically independent elements and $S\left(\mathfrak{h}_{\mathfrak{p}}\right)$ is a finite module over $\mathfrak{J}_{\mathfrak{h}_{\mathfrak{p}}}$. Let $\mathfrak{J}_{\mathfrak{h}}$ be the ring of restrictions to $\mathfrak{h}$ of elements of $\mathcal{F}$. Then $\mathfrak{J}_{\mathfrak{p}}$ coincides with ring of restrictions to $\mathfrak{h}_{\mathfrak{p}}$ of elements of $\mathcal{F}_{\mathfrak{b}}$. Let $\mathfrak{w}$ be the Weyl group of $\mathfrak{g}$ with respect to $\mathfrak{h}$ and $S(\mathfrak{h})$ 
the algebra of polynomial functions on $\mathfrak{h}$. Then Chevalley $\left(^{(5)}\right.$ has proved that every element of $S(\mathfrak{h})$ which is invariant under $\mathfrak{w}$ lies in $J_{\mathfrak{b}}$. It follows that every element $\phi \in S(\mathfrak{h})$ is integral over $J_{\mathfrak{b}}$ since it is a root of the polynomial $\prod_{s \in \mathfrak{w}}(T-s \phi)$ whose coefficients are in $\mathfrak{J}_{\mathfrak{b}}$. Moreover since $\mathfrak{w}$ is a finite group it follows from the theory of invariants that the ring of invariants of $\mathfrak{w}$ (in $S(\mathfrak{h})$ ) is finitely generated. Hence $\Im_{\mathfrak{b}}$ is a Noetherian ring. Now the operation of restriction from $\mathfrak{h}$ to $\mathfrak{h}_{\mathfrak{p}}$ is a homomorphism of $S(\mathfrak{h})$ onto $S\left(\mathfrak{h}_{\mathfrak{p}}\right)$ which maps $J_{\mathfrak{b}}$ onto $\Im_{\mathfrak{b}_{\mathfrak{p}}}$. Hence $\mathfrak{J}_{\mathfrak{b}_{\mathfrak{p}}}$ is a Noetherian ring and every element of $S\left(\mathfrak{h}_{\mathfrak{p}}\right)$ is integral over $J_{\mathfrak{b}_{p}}$. This shows that $J_{\mathfrak{b}_{\mathrm{p}}}$ contains $h$ algebraically independent elements. Furthermore since $S\left(\mathfrak{h}_{\mathfrak{p}}\right)$ may be obtained from $\mathfrak{J}_{\mathfrak{b}}$ by the adjunction of a finite number of elements which are integral over it, $S\left(\mathfrak{h}_{\mathfrak{p}}\right)$ is a finite module over $\Im_{b_{p}}$. Let $\Omega_{b_{p}}$ be the set of restrictions of elements of $\Omega$ to $\mathfrak{h}_{p}$. Then this restriction is a homomorphism of $\Omega$ onto $\Omega_{b_{p}}$. The ring $\Omega$ does not contain more than $h$ algebraically independent elements and contains $\Im_{\mathfrak{b}_{\mathfrak{p}}}$ which does contain $h$ algebraically independent elements. Since $\Omega$ and $\Omega_{b_{p}}$ are integral domains. this implies that the operation of restriction to $\mathfrak{h}_{\mathfrak{p}}$ induces an isomorphism of $\Omega$ onto $\Omega_{\mathfrak{b}_{\mathfrak{p}}}$. But since ${J_{\mathfrak{b}}}_{\mathfrak{p}} \subset \Omega_{\mathfrak{b}_{\mathfrak{p}}} \subset S\left(\mathfrak{h}_{\mathfrak{p}}\right)$ and since $\Im_{\mathfrak{b}_{\mathfrak{p}}}$ is Noetherian and $S\left(\mathfrak{h}_{\mathfrak{p}}\right)$ is a finite $\mathfrak{J}_{\mathfrak{b}_{\mathfrak{p}}}$-module, it follows (see van der Waerden $\left[16\right.$, vol. II, §99]) that $\Omega_{b_{p}}$ is also a finite $\Im_{b_{p}}$-module. Therefore in view of the above isomorphism $\Omega$ is a finite $J_{\mathfrak{p}}$-module.

2.3. The last step. We have now to prove that $\Im^{\prime}$ is a finite $\Omega$-module. For this we shall use the classical argument of Hilbert. Let $\mathfrak{A}$ be the $S(\mathfrak{p})$ module generated by $\Im^{\prime}$ (i.e. the set of linear combinations of elements of $\Im^{\prime}$ with coefficients in $S(\mathfrak{p})$ ). Since the ring $S(\mathfrak{p})$ is Noetherian and $S(\mathfrak{p}) \times W$ is a finite module over $S(\mathfrak{p}), \mathfrak{A}$ is a finite $S(\mathfrak{p})$-module and therefore $\mathfrak{A}$ $=\sum_{i=1}^{r} S(\mathfrak{p}) A_{i}\left(A_{i} \in \mathfrak{\Im}^{\prime}\right)$. We shall prove that $\mathfrak{\Im}^{\prime}=\sum_{i=1}^{r} \Omega A_{i}$. From Lemma 7, $S(\mathfrak{p}) \times W$ is the direct sum of $\mathfrak{Y}^{\prime}$ and the space $\mathfrak{M}$ spanned by the elements $\xi(X) A(X \in \mathfrak{l}$ and $A \in S(\mathfrak{p}) \times W)$. Similarly $S(\mathfrak{p})$ may be written on the direct sum of $\Omega$ and the space $\mathfrak{R}$ spanned by the elements $\rho(X) F(X \in \mathfrak{l}, F \in S(\mathfrak{p}))$. Let $A=\sum_{i=1}^{r} F_{i} A_{i}\left(F_{i} \in S(\mathfrak{p})\right)$ be an element of $\mathfrak{Y}^{\prime}$. Then $F_{i}=F_{i}^{\prime}+N_{i}$ when $F_{i}^{\prime} \in \Omega$ and $N_{i} \in \mathfrak{N}$. If $G \in S(\mathfrak{p}), \xi(X)\left(G A_{i}\right)=(\rho(X) G) A_{i}(X \in \mathfrak{l})$ since $\xi(X) A_{i}$ $=0$. Hence $(\rho(X) G) A_{i} \in \mathfrak{M}$ and therefore $N_{i} A_{i} \in \mathfrak{M}(1 \leqq i \leqq r)$. Now $F_{i}^{\prime} A_{i} \in \mathfrak{Y}^{\prime}$ and $A=\sum_{i=1}^{r} F_{i}^{\prime} A_{i}+\sum_{i=1}^{r} N_{i} A_{i}$. The sum $\Im^{\prime}+\mathfrak{M}$ being direct, it follows that $A=\sum_{i=1}^{r} F_{i}^{\prime} A_{i}$ and this proves our assertion. Theorem 1 is now rompletely proved.

\section{Part II. Well-behaved Functions on a Lie group}

4. Preliminary remarks. Let $G$ be a connected Lie group and $\mathfrak{E}$ a (complex) Banach space. For any bounded linear operator $A$ on $\mathfrak{E}$ we write $|A|=\sup _{|\psi| \leqq 1}|A \psi|(\psi \in \mathfrak{S})$. Let $I$ denote the unit operator on $\mathfrak{S}$. Then by a representation of $G$ on $\mathfrak{E}$ we mean a mapping $\pi$ which assigns to every

(5) Chevalley's results are not yet published. I am thankful to Professor Chevalley for being good enough to let me use them. 
$x \in G$ a bounded linear operator $\pi(x)$ on $\mathfrak{S}$ such that the following two conditions hold: G.

(1) $\pi(x y)=\pi(x) \pi(y)(x, y \in G)$ and $\pi(1)=I$ where 1 is the unit element of

(2) The mapping $\left({ }^{6}\right)(x, \psi) \rightarrow \pi(x) \psi(x \in G, \psi \in \mathfrak{S})$ is a continuous mapping of $G \times \mathfrak{L}$ into $\mathfrak{S}$.

It follows from the second condition that $|\pi(x)|$ is bounded on every compact set in $G$. Conversely assuming that (1) is fulfilled, it is easy to prove that if the mapping $x \rightarrow \pi(x) \psi(x \in G)$ is continuous at $x=1$ for every $\psi \in \mathfrak{S}$ and $|\pi(x)|$ is bounded on some neighbourhood of 1 in $G$, then $\pi$ is a representation.

Let $C_{c}^{\infty}(G)$ denote the set of all (complex-valued) functions on $G$ which are indefinitely differentiable everywhere and which vanish outside a compact set. Let $V$ be the subspace of $\mathfrak{S}$ spanned by all elements $\phi$ of the form

$$
\phi=\int_{G} f(x) \pi(x) \psi d x \quad\left(f \in C_{c}^{\infty}(G), \psi \in \mathfrak{S}\right)
$$

where $d x$ is the element of the left invariant Haar measure on $G$. Let $g_{0}$ be the Lie algebra of $G$ and $g$ its complexification. We denote by $\mathscr{B}$ the universal enveloping algebra of $\mathfrak{g}$. Gårding [5] has shown that for any $X \in \mathfrak{g}_{0}$ and $\phi \in V$ the limit

$$
\lim _{t \rightarrow 0} \frac{1}{t}\{\pi(\exp t X) \phi-\phi\}
$$

exists and lies in $V$ and if we denote this limit by $\pi_{V}(X) \phi$ we get a representation $\pi_{V}$ of $\mathfrak{g}$ (and therefore of $\mathfrak{B}$ ) on $V$. We shall call $V$ the Gårding subspace of $\mathfrak{E}$ and $\pi_{V}$ the Gårding representation of $\mathfrak{g}$ (or $\mathfrak{B}$ ). Unfortunately this representation has one serious shortcoming. If $U$ is a $\pi_{V}$-stable linear subspace of $V$, then its closure $\bar{U}$ is not necessarily invariant under $\pi(G)$. Thus one of the main links which connect representations of $G$ with those of $g_{0}$ in the finite-dimensional case is absent in the relationship between $\pi$ and $\pi_{V}$. Our principal objective now will be to restore this link by replacing the Gårding subspace by the space of all "well-behaved" elements in $\mathfrak{S}$.

5. Power series in a Banach space. Let $\left\{\psi_{\alpha}\right\}_{\alpha \in J}$ be an indexed set of elements in $\mathfrak{S}$. For any finite subset $F$ of $J$ let $s_{F}$ denote the sum $\sum_{\alpha \in F} \psi_{\alpha}$. We say that the series $\sum_{\alpha \in J} \psi_{\alpha}$ converges if there exists an element $\phi \in \mathfrak{G}$ such that for any $\epsilon>0$ we can find a finite subset $F_{0}$ of $J$ with the property that $\left|s_{F}-\phi\right| \leqq \epsilon$ whenever $F$ is a finite subset of $J$ containing $F_{0} . \phi$ is then called the sum of the series and we write $\phi=\sum_{\alpha \in J} \psi_{\alpha}$. (It is clear that $\phi$ if it exists is unique.) Moreover we say that the series converges absolutely if

(8) It can be shown that condition (2) can be replaced by the apparently weaker requirement that the mapping $x \rightarrow \pi(x) \psi(x \in G)$ be continuous for every $\psi \in \mathscr{\Phi}$. 
$\sum_{\alpha \in J}\left|\psi_{\alpha}\right|<\infty$. It is obvious that an absolutely convergent series is convergent.

Let $\sum_{e_{1}, \cdots, e_{n} \geq 0} \psi\left(e_{1}, \cdots, e_{n}\right) t_{1}^{\ell_{1}} \cdots t_{n}^{\ell_{n}}\left(\psi\left(e_{1}, \cdots, e_{n}\right) \in \mathfrak{S}\right)$ be a power series which converges for $t_{1}=a_{1}, \cdots, t_{n}=a_{n}\left(a_{i} \in C\right)$. Then it follows from the usual arguments that it converges absolutely for all $t_{1}, \cdots, t_{n}$ such that $\left|t_{i}\right|<\left|a_{i}\right|$. We shall say that the series( $\left.{ }^{7}\right) \sum_{(e)} \psi\left(e_{1}, \cdots, e_{n}\right) t_{1}^{e_{1}} \cdots t_{n}^{e_{n}}$ converges near the origin if it converges when $\left|t_{1}\right|, \cdots,\left|t_{n}\right|$ are all sufficiently small. It is clear that convergence near the origin implies absolute convergence for sufficiently small values of $\left|t_{1}\right|, \cdots,\left|t_{n}\right|$. Moreover a power series converges to zero near the origin if and only if all its coefficients are zero. Since the field of complex numbers is a Banach space, the above terminology is also applicable to ordinary power series with complex coefficients.

Lemma 14. Let $\sum_{e_{1}, \cdots, e_{n} \geq 0} \psi\left(e_{1}, \cdots, e_{n}\right) t_{1}^{e_{1}} \cdots t_{n}^{e_{n}}$ be a power series with coefficients in $\mathfrak{S}$ which converges near the origin. Let $g_{1}, \cdots, g_{n}$ be $n$ power series in $m$ variables $u_{1}, \cdots, u_{m}$ with complex coefficients which are all convergent near the origin and which take the values 0 at the origin (i.e. at $\left.u_{1}=u_{2}=\cdots=u_{m}=0\right)$. Let

$$
\sum_{(d)} a\left(e_{1}, \cdots, e_{n}, d_{1}, \cdots, d_{m}\right) u_{1}^{d_{1}} \cdots u_{m}^{d_{m}} \quad(a(e, d) \in C)
$$

be the power series expansion of $g_{1}^{e_{1}} \cdots g_{n}^{\varepsilon_{n}}$. Then the series

$$
\sum_{(e),(d)} \psi\left(e_{1}, \cdots, e_{n}\right) a\left(e_{1}, \cdots, e_{n}, d_{1}, \cdots, d_{n}\right) u_{1}^{d_{1}} \cdots u_{m}^{d_{m}}
$$

converges absolutely near the origin.

Choose $\epsilon>0$ such that the series $\sum_{(e)} \psi\left(e_{1}, \cdots, e_{n}\right) t_{1}^{e_{1}} \cdots t_{n}^{e_{n}}$ and the series $g_{1}, \cdots, g_{n}$ all converge absolutely if $\left|t_{i}\right| \leqq \epsilon,\left|u_{j}\right| \leqq \epsilon(1 \leqq i \leqq n, 1 \leqq j \leqq m)$. Let $g_{i}=\sum_{(d)} b_{i}\left(d_{1}, \cdots, d_{m}\right) u_{1}^{d_{1}} \cdots u_{m}^{d_{m}}\left(b_{i}(d) \in C, 1 \leqq i \leqq n\right)$. Since the constant term of $g_{i}$ is zero, it is possible to choose $\delta>0(\delta \leqq \epsilon)$ such that

$$
\sum_{(d)}\left|b_{i}\left(d_{1}, \cdots, d_{m}\right) u_{1}^{d_{1}} \cdots u_{m}^{d_{m}}\right| \leqq \epsilon
$$

if $\left|u_{j}\right| \leqq \delta(1 \leqq j \leqq m)$. Then it is clear that

$$
\begin{aligned}
& \sum_{(e),(d)}\left|\psi\left(e_{1}, \cdots, e_{n}\right) a\left(e_{1}, \cdots, e_{n}, d_{1}, \cdots, d_{m}\right) u_{1}^{d_{1}} \cdots u_{m}^{d_{m}}\right| \\
& \leqq \sum_{(e)}\left|\psi\left(e_{1}, \cdots, e_{n}\right)\right| \epsilon^{e_{1}+\cdots+e_{n}}<\infty
\end{aligned}
$$

provided $\left|u_{j}\right| \leqq \delta$. This proves the lemma.

( $\left.{ }^{7}\right)$ We shall of ten abbreviate $\left(e_{1}, \cdots, e_{n}\right)$ to $(e)$ and $\psi\left(e_{1}, \cdots, e_{n}\right)$ to $\psi(e)$. Similarly for other symbols. 
Lemma 15. Let $\sum_{e \geq 0} \psi(e) t^{\circ}(\psi(e) \in \mathfrak{S})$ be a power series which converges to $f(t)$ if $|t|<r(r>0)$. Then the power series $\sum_{e \geqq 1} \psi(e) e^{t-1}$ is convergent for $|t|$ $<r$ and its sum is equal to the limit

$$
\lim _{h \rightarrow 0} \frac{f(t+h)-f(t)}{h} \quad(h \in C,|t|<r) .
$$

It is clear that $\sum_{e \geqq 0}\left|\psi(e) t^{e}\right|$ converges if $|t|<r$ and from this it follows that the same is true of the series $\sum_{e \geq 1}\left|\psi(e) e t^{-1}\right|$. Therefore if $g(t)$ is the sum of the series $\sum_{e \geqq 1} \psi(e) e t^{e-1}(|t|<r)$, we have

$$
\left|\frac{f(t+h)-f(t)}{h}-g(t)\right| \leqq \sum_{e \geqq 0}|\psi(e)|\left|\frac{(t+h)^{e}-t^{e}}{h}-e t^{e-1}\right| .
$$

Choose $\delta$ so small $(\delta>0)$ that $|t|+2 \delta<r$. Then if $|h|<\delta / 2$,

$$
\left|\frac{(t+h)^{e}-t^{e}}{h}-e t^{e-1}\right|=\left|h \oint \frac{z^{e}}{(z-t)^{2}(z-t-h)} d z\right| \leqq|h| \frac{4 \pi}{\delta^{2}}(r-\delta)^{e}
$$

where $\Phi$ denotes complex integration on the circle $|z-t|=\delta$. Hence

$$
\left|\frac{f(t+h)-f(t)}{h}-g(t)\right| \leqq \frac{4 \pi}{\delta^{2}}|h| \sum_{e \geq 0}|\psi(e)|(r-\delta)^{e} .
$$

Since $\sum_{e \geq 0}|\psi(e)|(r-\delta)^{e}<\infty$ it follows that

$$
g(t)=\lim _{h \rightarrow 0} \frac{f(t+h)-f(t)}{h} .
$$

Put

$$
\frac{d}{d t} f(t)=\lim _{h \rightarrow 0} \frac{f(t+h)-f(t)}{h}
$$

Then

$$
\frac{d f(t)}{d t}=\sum_{e \geqq 1} e \psi(e) t^{e-1}
$$

Corollary. Let $\sum_{(e)} \psi\left(e_{1}, \cdots, e_{n}\right) t_{1}^{e_{1}} \cdots t_{n}^{e_{n}}\left(\psi\left(e_{1}, \cdots, e_{n}\right) \in \mathfrak{W}\right)$ be a power series which converges to $f\left(t_{1}, \cdots, t_{n}\right)=f(t)$ if $\left|t_{i}\right|\left\langle r_{i}\left(r_{i}>0,1 \leqq i \leqq n\right)\right.$. Then the series $\sum_{(e)} \psi\left(e_{1}, \cdots, e_{n}\right) \partial\left(t_{1}^{e_{1}} \cdots t_{n}^{e_{n}}\right) / \partial t_{i}$ converges in the same region to

$$
\frac{\partial f(t)}{\partial t_{i}}=\lim _{h \rightarrow 0} \frac{f\left(t_{1}, \cdots, t_{i}+h, \cdots, t_{n}\right)-f\left(t_{1}, \cdots, t_{n}\right)}{h} .
$$

We know that $\sum_{(e)} \psi\left(e_{1}, \cdots, e_{n}\right) t_{1}^{e_{1}} \cdots t_{n}^{e_{n}}$ converges absolutely for $\left|t_{j}\right|<r_{j}$. Hence the series obtained by collecting together the coefficients of 
$t_{i}^{e_{i}}$ is also absolutely convergent. Let $\psi^{\prime}\left(e_{i}\right)$ denote its sum. Then

$$
f(t)=\sum_{e_{i} \geqq 0} \psi^{\prime}\left(e_{i}\right) t_{i}^{e_{i}}
$$

and our assertion now follows from the above lemma.

Let $M$ be a real (complex) analytic manifold. A mapping $f$ of $M$ into a Banach space $\mathfrak{S}$ is called analytic (holomorphic) at a point $x_{0} \in M$ if for every coordinate system $\left(t_{1}, \cdots, t_{n}\right)$ at $x_{0}$ such that $t_{i}\left(x_{0}\right)=0$, there exists a neighbourhood $V$ of $x_{0}$ in $M$ (on which our coordinates are valid) and a power series $\sum_{e_{1}, \ldots, e_{n} \geq 0} \psi\left(e_{1}, \cdots, e_{n}\right) \tau_{1}^{e_{1}} \cdots \tau_{n}^{e_{n}}\left(\psi\left(e_{1}, \cdots, e_{n}\right) \in \mathfrak{E}\right)$ in $n$ variables $\tau_{1}, \cdots, \tau_{n}$ such that this series converges to $f(x)$ at $\tau_{i}=t_{i}(x)$, $1 \leqq i \leqq n(x \in V)$. It follows easily from Lemma 14 that if the above condition is fulfilled for one coordinate system $\left(t_{1}, \cdots, t_{n}\right)$, then it necessarily holds for all coordinate systems $\left(u_{1}, \cdots, u_{n}\right)$ at $x_{0}$ such that $u_{i}\left(x_{0}\right)=0,1 \leqq i \leqq n$.

Lemma 16. Let $M$ and $M^{\prime}$ be real analytic manifolds and $g$ a mapping of $M^{\prime}$ into $M$ which is analytic at a point $x_{0}^{\prime} \in M^{\prime}$. Let $x_{0}=g\left(x_{0}^{\prime}\right)$ and let $f$ be a mapping of $M$ into $\mathfrak{S}$ which is analytic at $x_{0}$. Then the mapping $f \circ g$ of $M^{\prime}$ into $\mathfrak{S}$ is analytic at $x_{0}^{\prime}$.

This again is an immediate consequence of Lemma 14.

Lemma 17. Let $f$ be a mapping of $M$ into $\mathfrak{S}$ analytic at $x_{0}$. Let $u$ be a (complexvalued) function on $M$ which is analytic at $x_{0}$. Then the mapping uf is analytic at $x_{0}$.

Choose a coordinate system $\left(t_{1}, \cdots, t_{n}\right)$ at $x_{0}$ such that $t_{i}\left(x_{0}\right)=0,1 \leqq i \leqq n$. Then if $x$ lies in a suitable neighbourhood $V$ of $x_{0}$,

$$
\begin{array}{lr}
f(x)=\sum_{(e)} \psi\left(e_{1}, \cdots, e_{n}\right) t_{1}^{e_{1}}(x) \cdots t_{n}^{e_{n}}(x) & (\psi(e) \in \mathfrak{E}), \\
u(x)=\sum_{(e)} a\left(e_{1}, \cdots, e_{n}\right) t_{1}^{e_{1}}(x) \cdots t_{n}^{e_{n}}(x) & (a(e) \in C)
\end{array}
$$

both series being absolutely convergent. Hence

$$
\sum_{(o),(d)} \psi\left(e_{1}, \cdots, e_{n}\right) a\left(d_{1}, \cdots, d_{n}\right) t_{1}^{e_{1}+d_{1}}(x) \cdots t_{n}^{e_{n}+d_{n}}(x)
$$

converges absolutely to $u(x) f(x)$ for $x \in V$. From this the lemma follows.

Lemma 18. Let $f$ be a mapping of $M$ into $\mathfrak{S}$ which is analytic at $x_{0}$ and let $A$ be a continuous linear mapping of $\mathfrak{S}$ into a Banach space $\mathfrak{S}^{\prime}$. Then the mapping $A$ of of $M$ into $\mathfrak{S}^{\prime}$ is analytic at $x_{0}$.

Let $\phi$ be the sum of a convergent series $\sum_{\alpha \in J} \psi_{\alpha}$ in $\mathfrak{S}$. Since $A$ is linear and continuous, the series $\sum_{\alpha \in J} A \psi_{\alpha}$ is convergent in $\mathfrak{Q}^{\prime}$ and its sum is $A \phi$. The assertion in the lemma is an immediate consequence of this fact. 
Let $f$ be a mapping of $M$ into $\mathfrak{S}$ which is analytic at $x_{0}$ and let $X_{0}$ be a vector $\left(^{8}\right)$ in the tangent space of $M$ at $x_{0}$. Let $\left(t_{1}, \cdots, t_{n}\right)$ be a coordinate system at $x_{0}$ such that $t_{i}\left(x_{0}\right)=0$ and let

$$
f(x)=\sum_{(e)} \psi\left(e_{1}, \cdots, e_{n}\right) t_{1}^{e_{1}}(x) \cdots t_{n}^{i_{n}}(x) \quad\left(\psi\left(e_{1}, \cdots, e_{n}\right) \in \mathfrak{W}\right)
$$

for $x$ sufficiently near $x_{0}$. Then we put

$$
X_{0} f=\sum_{1 \leqq i \geqq n} \psi_{i}\left(X_{0} t_{i}\right)
$$

where $\psi_{i}$ is the value of $\psi\left(e_{1}, \cdots, e_{n}\right)$ for $e_{i}=1$ and $e_{j}=0, j \neq i$. It is easy to verify that the value of $X_{0} f$ does not depend on the particular choice of the coordinate system $\left(t_{1}, \cdots, t_{n}\right)$ used in its definition.

LemMA 19. Let $f$ and $X_{0}$ be as above and $\phi$ a continuous linear function on S. Then the function $\phi(f(x))$ is analytic at $x_{0}$ and $X_{0}(\phi(f))=\phi\left(X_{0} f\right)$.

$\phi$ is a continuous linear mapping of $\mathfrak{S}$ into $C$. Hence from Lemma 18, $\phi(f(x))$ is analytic at $x_{0}$. Moreover $\phi(f(x))=\sum_{(0)} \phi\left(\psi\left(e_{1}, \cdots, e_{n}\right)\right) t_{1}^{e_{1}}(x) \cdots$ $t_{n}^{\ln }(x)$ if $x$ is sufficiently near $x_{0}$. Hence

$$
X_{0}(\phi(f))=\sum_{1 \leqq i \leqq n} \phi\left(\psi_{i}\right)\left(X_{0} t_{i}\right)=\phi\left(X_{0} f\right) .
$$

Lemma 20. Suppose $M^{\prime}$ and $M$ are real analytic manifolds and $g$ is a mapping of $M^{\prime}$ into $M$ which is analytic at $x_{0}^{\prime} \in M^{\prime}$. Put $x_{0}=g\left(x_{0}^{\prime}\right)$ and let $f$ be a mapping of $M$ into $\mathfrak{S}$ which is analytic at $x_{0}$. Then if $X_{0}^{\prime}$ is a vector in the tangent space of $M^{\prime}$ at $x_{0}^{\prime}$,

$$
X_{0}^{\prime}(f \circ g)=\left(d g X_{0}^{\prime}\right) f
$$

where $d g$ is the differential of $g$.

This follows without difficult from Lemmas 16 and 14.

We regard the field $R$ of real numbers as a real analytic manifold in the usual way. For a given $t_{0} \in R$ let $T_{0}$ be the vector in the tangent space of $R$ at $t_{0}$ such that $T_{0} g=\{d g(t) / d t\}_{t-t_{0}}$ for any function $g$ which is analytic at $t_{0}$.

Lemma 21. Let $f$ be a mapping of $R$ into $\mathfrak{S}$ which is analytic at $t_{0}$. Then

$$
T_{0} f=\lim _{h \rightarrow 0} \frac{1}{h}\left\{f\left(t_{0}+h\right)-f\left(t_{0}\right)\right\}=\left(\frac{d f}{d t}\right)_{t_{0}} .
$$

Put $h(t)=t-t_{0}(t \in R)$. Then $h\left(t_{0}\right)=0$ and $h$ can be chosen as a coordinate on $R$. Since $f$ is analytic at $t_{0}$ we can write

$$
f(t)=\sum_{e \geq 0} \psi(e) h^{e}(t)
$$

(3) We use here and in what follows the terminology of Chevalley [4, Chap. III]. 
for $t$ sufficiently near $t_{0}$. Hence

$$
\frac{f(t)-f\left(t_{0}\right)}{h(t)}=\sum_{e \geqq 1} \psi(e) h^{e-1}(t)
$$

and therefore

$$
\lim _{t \rightarrow 0} \frac{f(t)-f\left(t_{0}\right)}{h(t)}=\psi(1)=T_{0} f .
$$

This proves the lemma.

CoROLlary. Let $g$ be a mapping of $R$ in $M$ which is analytic at $t_{0}$ and $f$ a mapping of $M$ into $\mathfrak{S}$ which is analytic at $x_{0}=g\left(t_{0}\right)$. Then if $X_{0}=d g T_{0}$,

$$
X_{0} f=\left(\frac{d}{d t} f(g(t))\right)_{t=t_{0}}=\lim _{h \rightarrow 0} \frac{f\left(g\left(t_{0}+h\right)\right)-f\left(g\left(t_{0}\right)\right)}{h} .
$$

This follows immediately from Lemmas 20 and 21.

Let $f$ be a mapping of a real analytic manifold $M$ into $\mathfrak{S}$. We say $f$ is analytic if it is analytic at every point of $M$.

LeMma 22. Let $f$ be an analytic mapping of $M$ into $\mathfrak{S}$ and $X$ an analytic infinitesimal transformation on $M$. Then the mapping $X f$ (i.e. $x \rightarrow X(x) f, x \in M$ ) is also analytic. Moreover if $Y$ is another infinitesimal transformation and $Z=[X, Y]=X Y-Y X$, then

$$
Z f=X \cdot Y f-Y \cdot X f
$$

Let $x_{0} \in M$ and let $\left(t_{1}, \cdots, t_{n}\right)$ be a coordinate system at $x_{0}$ such that $t_{i}\left(x_{0}\right)=0,1 \leqq i \leqq n$. Then there exists an open neighbourhood $U$ of $x_{0}$ (on which the system $\left(t_{1}, \cdots, t_{n}\right)$ is valid) and functions $u_{1}, \cdots, u_{n}$ which are analytic on $U$ such that $X=\sum_{1 \leqq i \leqq n} u_{i} \partial / \partial t_{i}$ on $U$. Moreover we may choose $U$ so small that we have the expansion

$$
f(x)=\sum_{(e)} \psi\left(e_{1}, \cdots, e_{n}\right) t_{1}^{e_{1}}(x) \cdots t_{n}^{e_{n}}(x) \quad(\psi(e) \in \mathfrak{S}, x \in U) .
$$

It follows from the above corollary that

$$
\begin{aligned}
\left(\frac{\partial f}{\partial t_{i}}\right)(x)= & \lim _{h \rightarrow 0} \frac{1}{h}\left\{\sum_{(e)} \psi\left(e_{1}, \cdots, e_{n}\right) t_{1}^{e_{1}}(x) \cdots\left(t_{i}(x)+h\right)^{e_{i}} \cdots t_{n}^{e_{n}}(x)\right. \\
& \left.-\sum_{(e)} \psi\left(e_{1}, \cdots, e_{n}\right) t_{1}^{e_{1}}(x) \cdots t_{i}^{e_{i}}(x) \cdots t_{n}^{e_{n}}(x)\right\} \\
= & \sum_{(e)} \psi\left(e_{1}, \cdots, e_{n}\right)\left\{\frac{\partial}{\partial t_{i}}\left(t_{1}^{e_{1}} \cdots t_{n}^{e_{n}}\right)\right\}_{t_{j}-t_{j}(x)}
\end{aligned}
$$


in view of the corollary to Lemma 15 . This proves that $\partial f / \partial t_{i}$ is analytic at $x_{0}$. Since the functions $u_{i}$ are analytic at $x_{0}$ we conclude from Lemma 17 that $X f$ is analytic at $x_{0}$.

Now we come to the second part. Let $\phi$ be a continuous linear function on $\mathfrak{S}$ and $g$ an analytic mapping of $M$ into $\mathfrak{S}$. Then from Lemma 19 the function $\phi_{g}: x \rightarrow \phi(g(x))$ is an analytic function on $M$ and $X \phi_{g}=\phi_{X_{g}}$ for any analytic linear transformation $X$ on $M$. From this it follows that $\phi_{Z f}=\phi_{X Y f}$ $-\phi_{Y X f}$. Now put $g=Z f-(X Y f-Y X f)$. Then $\phi(g(x))=0$. Since this is true for every $\phi$ it follows from the Hahn-Banach Theorem that $Z f=X Y f-Y X f$.

6. Banach spaces of functions. Let $L$ be a closed finite interval on the real line and $t \rightarrow \psi_{t}$ a continuous mapping of $L$ into a Banach space $\mathfrak{S}$. Then $\left|\psi_{t}\right|$ is a continuous function on $L$ and $\int_{L}\left|\psi_{t}\right| d t<\infty$. Therefore the integral $\int_{L} \psi_{t} d t$ is defined and for any bounded linear function $\alpha$ on $\mathfrak{S}, \alpha\left(\int_{L} \psi_{t} d t\right)$ $=\int_{L} \alpha\left(\psi_{t}\right) d t$. Let $D$ be a domain (i.e. an open connected set) in the complex plane and $z \rightarrow \psi_{z}$ a continuous mapping of $D$ into $\mathfrak{W}$. Let $\Gamma$ be a rectifiable curve in $D$. Then it is clear that the complex integral $\int_{\Gamma} \psi_{z} d z$ exists and $\alpha\left(\int_{\Gamma} \psi_{z} d z\right)$ $=\int_{\Gamma} \alpha\left(\psi_{z}\right) d z$.

Let $E$ be a locally compact Hausdorff space with a (regular) positive measure $\mu$ given on it. Let $\mathfrak{S}$ denote the Banach space of all $\mu$-summable functions on $E$.

Lemma 23. Let $D$ be a domain in $C^{n}$ and $\left(z_{1}, \cdots, z_{n}, x\right) \rightarrow f\left(z_{1}, \cdots, z_{n} ; x\right)$ a continuous function on $D \times E$ which satisfies the following two conditions:

(a) there exists a $\mu$-summable function $g$ on $E$ such that $\left|f\left(z_{1}, \cdots, z_{n} ; x\right)\right|$ $\leqq|g(x)|$ for all $\left(z_{1}, \cdots, z_{n}, x\right) \in D \times E$;

(b) for each $x \in E$ the function $\left(z_{1}, \cdots, z_{n}\right) \rightarrow f\left(z_{1}, \cdots, z_{n} ; x\right)$ is holomorphic on $D$.

Let $\psi\left(z_{1}, \cdots, z_{n}\right)$ be the element of $\mathfrak{S}$ represented by the function $x \rightarrow f\left(z_{1}, \cdots, z_{n} ; x\right)$. Then the mapping $\left(z_{1}, \cdots, z_{n}\right) \rightarrow \psi\left(z_{1}, \cdots, z_{n}\right)$ is a holomorphic mapping of $D$ into $\mathfrak{S}$.

Let $\left(z_{1}, \cdots, z_{n}\right) \in D$. Given $\epsilon>0$ and a compact set $K$ in $E$ we can find $\delta>0$ such that if $\max _{i}\left|h_{i}\right|<\delta$, then $\left(z_{1}+h_{1}, \cdots, z_{n}+h_{n}\right) \in D$ and

$$
\left|f\left(z_{1}+h_{1}, \cdots, z_{n}+h_{n} ; x\right)-f\left(z_{1}, \cdots, z_{n} ; x\right)\right| \leqq \epsilon
$$

for $x$ in $K$. Since $|g|$ is $\mu$-summable we can choose $K$ such that $\int_{E-K}|g| d \mu \leqq \epsilon$. Hence

$$
\begin{aligned}
\mid \psi\left(z_{1}+h_{1}, \cdots, z_{n}\right. & \left.+h_{n}\right)-\psi\left(z_{1}, \cdots, z_{n}\right) \mid \\
& =\int\left|f\left(z_{1}+h_{1}, \cdots, z_{n}+h_{n} ; x\right)-f\left(z_{1}, \cdots, z_{n} ; x\right)\right| d \mu \\
& \leqq \epsilon \int_{K} d \mu+2 \int_{E-K}|g| d \mu \leqq(\mu(K)+2) \epsilon
\end{aligned}
$$


if $\left|h_{i}\right|<\delta$. This shows that the mapping $\left(z_{1}, \cdots, z_{n}\right) \rightarrow \psi\left(z_{1}, \cdots, z_{n}\right)$ is a continuous mapping of $D$ into $\mathfrak{S}$.

Now let $\left(a_{1}, \cdots, a_{n}\right)$ be any point of $D$. Choose positive real numbers $r_{1}, \cdots, r_{n}$ such that the polycylinder $P$ defined by the conditions $\left|z_{i}-a_{i}\right|$ $\leqq r_{i}(1 \leqq i \leqq n)$ lies in $D$. Let $\left(z_{1}, \cdots, z_{n}\right)$ be any interior point of $P$. Put

$$
\phi\left(z_{1}, \cdots, z_{n}\right)=\left(\frac{1}{2 \pi(-1)^{1 / 2}}\right)^{n} \oint_{1} \cdots \oint_{n} \frac{\psi\left(\zeta_{1}, \cdots, \zeta_{n}\right)}{\left(\zeta_{1}-z_{1}\right) \cdots\left(\zeta_{n}-z_{n}\right)} d \zeta_{1} \cdots d \zeta_{n}
$$

where $\mathscr{S}_{K}$ denotes complex integration on the circle $\left|\zeta_{k}-a_{k}\right|=r_{k}$. For any measurable set $X$ in $E$ and a $\mu$-summable function $F$ on $E$ put

$$
\alpha_{X}(F)=\int_{X} F d \mu .
$$

Then $\alpha_{X}$ may clearly be regarded as a bounded linear function on $\mathfrak{W}$. Moreover $\alpha_{X}(F)=0$ for all $X$ implies $F=0 \mu$-almost everywhere. Therefore if $\alpha_{X}(\psi)=0(\psi \in \mathfrak{S})$ for all $X$ then $\psi=0$. Now

$\alpha_{X}\left(\phi\left(z_{1}, \cdots, z_{n}\right)\right)$

$$
=\left(\frac{1}{2 \pi(-1)^{1 / 2}}\right)^{n} \oint_{1} \cdots \oint_{n} \frac{\alpha_{X}\left(\psi\left(\zeta_{1}, \cdots, \zeta_{n}\right)\right)}{\left(\zeta_{1}-z_{1}\right) \cdots\left(\zeta_{n}-z_{n}\right)} d \zeta_{1} \cdots d \zeta_{n} .
$$

But $\alpha_{X}\left(\psi\left(\zeta_{1}, \cdots, \zeta_{n}\right)\right)=\int_{X} f\left(\zeta_{1}, \cdots, \zeta_{n} ; x\right) d \mu$ and therefore

$$
\left|\alpha_{X}\left(\psi\left(\zeta_{1}, \cdots, \zeta_{n}\right)\right)\right| \leqq \int|g| d \mu .
$$

Hence it is clear that

$$
\begin{aligned}
\alpha_{X}\left(\phi\left(z_{1}, \cdots, z_{n}\right)\right) & \\
& =\left(\frac{1}{2 \pi(-1)^{1 / 2}}\right)^{n} \int_{X} d \mu \oint_{1} \cdots \oint_{n} \frac{f\left(\zeta_{1}, \cdots, \zeta_{n} ; x\right)}{\left(\zeta_{1}-z_{1}\right) \cdots\left(\zeta_{n}-z_{n}\right)} d \zeta_{1} \cdots d \zeta_{n} \\
& =\int_{X} f\left(z_{1}, \cdots, z_{n} ; x\right) d \mu
\end{aligned}
$$

by Cauchy's Theorem. Therefore

$$
\alpha_{X}\left(\phi\left(z_{1}, \cdots, z_{n}\right)\right)=\alpha_{X}\left(\psi\left(z_{1}, \cdots, z_{n}\right)\right) .
$$

This being true for all $X$ we conclude that

$$
\begin{aligned}
\psi\left(z_{1}, \cdots, z_{n}\right) & =\phi\left(z_{1}, \cdots, z_{n}\right) \\
& =\left(2 \pi(-1)^{1 / 2}\right)^{-n} \oint_{1} \cdots \oint_{n} \frac{\psi\left(\zeta_{1}, \cdots, \zeta_{n}\right)}{\left(\zeta_{1}-z_{1}\right) \cdots\left(\zeta_{n}-z_{n}\right)} d \zeta_{1} \cdots d \zeta_{n} .
\end{aligned}
$$

It follows immediately from this formula, by the classical argument, that 
there exists a power series in $\left(z_{1}-a_{1}\right), \cdots,\left(z_{n}-a_{n}\right)$ with coefficients in $\mathfrak{E}$ which converges in the interior of $P$ to $\psi\left(z_{1}, \cdots, z_{n}\right)$. Therefore the mapping $\left(z_{1}, \cdots, z_{n}\right) \rightarrow \psi\left(z_{1}, \cdots, z_{n}\right)$ is holomorphic at $\left(a_{1}, \cdots, a_{n}\right)$.

7. Well-behaved vectors in a representation space. Let $\pi$ be a representation of a connected Lie group $G$ on a Banach space $\mathfrak{S}$. We shall say that an element $\psi \in \mathfrak{S}$ is well-behaved (under $\pi$ ) if the mapping $f: x \rightarrow \pi(x) \psi$ is an analytic mapping of $G$ into $\mathfrak{S}$. For any $y \in G$ let $\phi_{y}$ denote the mapping $x \rightarrow y^{-1} x(x \in G)$. Then it is easy to verify that $\pi(y) \circ f \circ \phi_{y}=f$. Since $\phi_{y}$ is an analytic isomorphism of $G$ with itself it follows from Lemmas 16 and 18 that if $f$ is analytic at $x=1$ then $f=\pi(y) \circ f \circ \phi_{y}$ is analytic at $y$. Therefore in order that $f$ be analytic it is sufficient that it should be analytic at 1 .

Let $W$ be the set of all elements in $\mathfrak{S}$ which are well-behaved under $\pi$. Clearly $W$ is a linear subspace of $\mathfrak{S}$. Moreover since for a fixed $y \in G$ the mapping $x \rightarrow x y$ is an analytic mapping of $G$, it follows that if $\psi \in W$ then $\pi(y) \psi \in W$. Let $\mathrm{g}_{0}$ be the Lie algebra of $G$. For any $\psi \in W$ and $X \in \mathfrak{g}_{0}$ consider the mapping $t \rightarrow \pi(\exp t X) \psi(t \in R)$. Clearly this mapping is analytic and therefore from Lemma 21 the limit

$$
\lim _{t \rightarrow 0} \frac{1}{t}\{\pi(\exp t X) \psi-\psi\}
$$

exists. We denote this limit by $\pi_{W}(X) \psi$. Let $f$ be the mapping $x \rightarrow \pi(x) \psi$ and $g$ the mapping $x \rightarrow \pi(x) \pi_{W}(X) \psi$. Then it follows from Lemmas 20 and 21 that $g=X f$ and so $g$ is analytic (Lemma 22). Hence $\pi_{W}(X) \psi \in W$. Thus we get a linear transformation $\pi_{W}(X)$ of $W$ into itself. Moreover if $[X, Y]=Z$ $\left(X, Y, Z \in g_{0}\right)$ we know from Lemma 22 that $Z f=X \cdot Y f-Y \cdot X f$ and therefore $\pi_{W}([X, Y])=\pi_{W}(X) \pi_{W}(Y)-\pi_{W}(Y) \pi_{W}(X)$. This shows that the mapping $X \rightarrow \pi_{W}(X)$ is a representation of $g_{0}$ on $W$. Let $g_{0}$ be the complexification of $g$ and $\mathfrak{B}$ the universal enveloping algebra of $\mathfrak{g}$. We denote by $\pi_{W}$ the representation of $\mathfrak{B}$ on $W$ which coincides on $\mathfrak{g}_{0}$ with this representation.

Since $g_{0}$ is a vector space of finite dimension over $R$ we may regard it as a real analytic manifold in the obvious way. Then the most important property of well-behaved elements may be expressed as follows.

THeOREM 2. Let $\psi$ be an element in $W$. Then there exists a neighbourhood $V$ of zero in $\mathrm{g}_{0}$ such that the series $\sum_{m \geqq 0}(1 / m !) \pi_{W}\left(X^{m}\right) \psi$ converges to $\pi(\exp X) \psi$ for $X \in V$.

Let $\left(t_{1}, \cdots, t_{n}\right)$ be the Cartesian coordinate system in $g_{0}$ corresponding to a base $\left(X_{1}, \cdots, X_{n}\right)$ so that $X=\sum_{i=1}^{n} t_{i}(X) X_{i}\left(X \in g_{0}\right)$. Since the mapping $X \rightarrow \pi(\exp X) \psi$ is an analytic mapping of $\mathfrak{g}_{0}$ into $\mathfrak{S}$, we can find a neighbourhood $V$ of zero in go such that

$$
\pi(\exp X) \psi=\sum_{(\Theta)} \psi\left(e_{1}, \cdots, e_{n}\right) t_{1}^{e_{1}}(X) \cdots t_{n}^{e_{n}}(X)
$$


$\left(\psi\left(e_{1}, \cdots, e_{n}\right) \in \mathfrak{G}, X \in V\right)$, the series being absolutely convergent. We shall now prove that

$$
\sum_{e_{1}+\cdots+e_{n}=m} \psi\left(e_{1}, \cdots, e_{n}\right) t_{1}^{e_{1}}(X) \cdots t_{n}^{e_{n}}(X)=\frac{1}{m !} \pi_{W}\left(X^{m}\right) \psi \quad\left(X \in g_{0}\right) .
$$

For a fixed $X \in \mathfrak{g}_{0}$ choose $r>0$ so small that $u X \in V$ whenever $|u| \leqq r(u \in R)$. Then

$$
\pi(\exp u X) \psi=\sum_{m \geqq 0} \psi_{m} u^{m} \quad(|u|<r)
$$

where $\psi_{m}=\sum_{e_{1}+\ldots+e_{n}=m} \psi\left(e_{1}, \cdots, e_{n}\right) \ell_{1}^{\ell_{1}}(X) \cdots t_{n}^{e_{n}}(X)$. We shall now prove by induction on $m$ that

$$
\pi(\exp u X) \pi_{W}\left(X^{m}\right) \psi=\sum_{\mu \geq m} \psi_{\mu}\left(\frac{d^{m}}{d u^{m}} u^{\mu}\right) \quad(|u|<r) .
$$

This is true for $m=0$. Now

$$
\begin{aligned}
\pi_{W}\left(X^{m+1}\right) \psi & =\pi_{W}(X) \pi_{W}\left(X^{m}\right) \psi \\
& =\lim _{s \rightarrow 0} \frac{1}{s}\left\{\pi(\exp s X) \pi_{W}\left(X^{m}\right) \psi-\pi_{W}\left(X^{m}\right) \psi\right\} .
\end{aligned}
$$

Since $\pi(\exp u X)$ is a bounded operator,

$$
\pi(\exp u X) \pi_{W}\left(X^{m+1}\right) \psi=\lim _{s \rightarrow 0} \frac{1}{s}[\pi(\exp (s+u) X)-\pi(\exp u X)] \psi^{\prime}
$$

where $\psi^{\prime}=\pi_{W}\left(X^{m}\right) \psi$. But

$$
\pi(\exp u X) \psi^{\prime}=\sum_{\mu \geq m} \psi_{\mu}\left(\frac{d^{m}}{d u^{m}} w^{\mu}\right) \quad(|u|<r)
$$

by our induction hypothesis. Hence

$$
\pi(\exp u X)_{\pi_{W}}\left(X^{m+1}\right) \psi=\sum_{\mu \geqq m+1} \psi_{\mu} \frac{d^{m+1}}{d u^{m+1}} u^{\mu} \quad(|u|<r)
$$

from Lemma 21 and so our assertion is proved. Now if we put $u=0$ we get

$$
\pi_{W}\left(X^{m}\right) \psi=m ! \psi_{m}=m ! \sum_{e_{1}+\cdots+e_{n}=m} \psi\left(e_{1}, \cdots, e_{n}\right) t_{1}^{e_{1}}(X) \cdots t_{n}^{\epsilon_{n}}(X) .
$$

Therefore if $X \in V$

$$
\pi(\exp X) \psi=\sum_{m \geqq 0} \frac{1}{m !} \pi_{W}\left(X^{m}\right) \psi .
$$

Corollary. Let $\psi$ be a well-behaved element and let $\mathrm{Cl}\left(\pi_{W}(\mathfrak{B}) \psi\right)$ denote the 
closure of $\pi_{W}(\mathfrak{B}) \psi$ in $\mathfrak{S}$. Then $\mathrm{Cl}\left(\pi_{W}(\mathfrak{B}) \psi\right)$ is invariant under $\pi(G)$.

Let $\psi_{0}$ be any element in $U=\pi_{W}(\mathfrak{B}) \psi$ and consider any continuous linear function $\phi$ on $\mathfrak{W}$ which vanishes on $U$. Since $\psi_{0} \in W$ the function $x \rightarrow \phi\left(\pi(x) \psi_{0}\right)$ $(x \in G)$ is an analytic function on $G$. On the other hand in view of the above theorem there exists a neighbourhood $V$ of zero in $g_{0}$ such that

$$
\pi(\exp X) \psi_{0}=\sum_{m \geqq 0} \frac{1}{m !} \pi_{W}\left(x^{m}\right) \psi_{0}
$$

Therefore

$$
\phi\left(\pi(\exp X) \psi_{0}\right)=\sum_{m \geq 0} \frac{1}{m !} \phi\left(\pi_{W}\left(X^{m}\right) \psi_{0}\right) .
$$

But $\pi_{W}\left(X^{m}\right) \psi_{0} \in U$ and therefore $\phi\left(\pi_{W}\left(X^{m}\right) \psi_{0}\right)=0$. Hence $\phi\left(\pi(\exp X) \psi_{0}\right)=0$ for $X \in V$ and therefore $\phi\left(\pi(x) \psi_{0}\right)$ vanishes on a neighbourhood of 1 in $G$. But since it is an analytic function on $G$ it follows that $\phi\left(\pi(x) \psi_{0}\right)=0$ for all $x \in G$. Keeping $x$ fixed and varying $\phi$ we deduce from the Hahn-Banach theorem that $\pi(x) \psi_{0} \in \mathrm{Cl}(U)$. This proves that $\pi(x) U \subset \mathrm{Cl}(U)(x \in G)$ and therefore by continuity $\pi(x) \mathrm{Cl}(U) \subset \mathrm{Cl}(U)$.

8. Well-behaved functions. Let $\pi$ be a representation of $G$ on a Banach space $\mathfrak{S}$. Then $|\pi(x)|=\sup _{|\psi| \leqq 1}|\pi(x) \psi|(\psi \in \mathfrak{S})$ is a semi-continuous function on $G$ and therefore it is measurable (with respect to the Haar measure). Let $L(G, \pi)$ denote the space of all (complex-valued) measurable functions $f$ on $G$ such that

$$
\|f\|=\int_{G}|f(x)||\pi(x)| d x<\infty
$$

where $d x$ is the element of the left-invariant Haar measure on $G$. Then with respect to the norm \|\|$, L(G, \pi)$ is a Banach space and we get a representation $\lambda$ of $G$ on $L(G, \pi)$ if we define $\lambda(y) f$ to be the function whose value at $x$ is $f\left(y^{-1} x\right)(x, y \in G, f \in L(G, \pi))$. In fact,

$$
\|\lambda(y) f\|=\int\left|f\left(y^{-1} x\right)\right||\pi(x)| d x=\int|f(x)||\pi(y x)| d x \leqq|\pi(y)|\|f\|
$$

and therefore $\lambda(y)$ is a bounded operator and

$$
\|\lambda(y)\|=\sup _{\|f\|=1}\|\lambda(y) f\| \leqq|\pi(y)| .
$$

This shows that $\|\lambda(y)\|$ remains bounded on a compact set. Therefore, in order to prove that $\lambda$ is a representation, it is sufficient to show that $\lim _{x \rightarrow 1}\left\|\lambda(x) f_{0}-f_{0}\right\|=0$ for any $f_{0} \in L(G, \pi)$. Let $V$ be a compact neighbourhood of 1 in $G$ and $m$ an upper bound for $\|\lambda(x)\|$ on $V$. Given any $\epsilon>0$ we can choose a continuous function $g$ which vanishes outside a compact set such 
that $\left\|f_{0}-g\right\| \leqq \epsilon$. Since $|\pi(x)|$ remains bounded on a compact set, it is clear that we can find a neighbourhood $U$ of $1(U \subset V)$ such that

$$
\|\lambda(x) g-g\|=\int\left|g\left(x^{-1} y\right)-g(y)\right||\pi(y)| d y \leqq \epsilon .
$$

Then

$$
\begin{aligned}
\left\|\lambda(x) f_{0}-f_{0}\right\| & =\left\|\lambda(x)\left(f_{0}-g\right)-\left(f_{0}-g\right)+(\lambda(x) g-g)\right\| \\
& \leqq\|\lambda(x)\| \epsilon+\epsilon+\epsilon \leqq(m+2) \epsilon
\end{aligned}
$$

This proves that $\lim _{x \rightarrow 1}\left\|\lambda(x) f_{0}-f_{0}\right\|=0$ and therefore $\lambda$ is indeed a representation.

Let $\mathfrak{O}$ be the Banach space of all bounded linear operators on $\mathfrak{S}$ with the norm $|A|=\sup _{|\psi|} \leqq 1 \quad|A \psi| \quad(A \in \mathcal{O}, \psi \in \mathfrak{G})$. If $f \in L(G, \pi)$, the integral $\int_{G} f(x) \pi(x) d x$ has a well-defined meaning in $\mathfrak{O}$ since $\int_{G}|f(x) \pi(x)| d x=\|f\|<\infty$. Put $T_{f}=\int f(x) \pi(x) d x$. Then the mapping $T: f \rightarrow T_{f}$ is a continuous linear mapping of $L(G, \pi)$ into $\mathfrak{D}$ since $\left|T_{f}\right| \leqq\|f\|$. Now let $\mathfrak{B}_{\pi}$ denote the set of all elements in $L(G, \pi)$ which are well-behaved under $\lambda$. Then for any $\psi \in \mathfrak{E}$,

$$
\pi(x) T_{f} \psi=\int_{G} f(y) \pi(x y) \psi d y=\int_{G} f\left(x^{-1} y\right) \pi(y) \psi d y=T_{\lambda(x) f} \psi .
$$

But the mapping $x \rightarrow \lambda(x) f$ is analytic and $T$ is linear and continuous. Similarly $A \rightarrow A \psi(A \in \mathcal{O})$ is a continuous linear mapping of $\mathfrak{D}$ into $\mathfrak{Q}$ since $|A \psi| \leqq|A||\psi|$. Hence $x \rightarrow T_{\lambda(x) f} \psi=\pi(x) T_{f} \psi$ is an analytic mapping of $G$ into $\mathfrak{S}$ (Lemma 18). Thus we have the following result.

Lemma 24. Let $f \in \mathfrak{B}_{\pi}$ and $\psi \in \mathfrak{S}$. Then $\int_{G} f(x) \pi(x) \psi d x$ is a well-behaved element of $\mathfrak{S}$ (with respect to $\pi$ ).

We shall now investigate the question of approximating arbitrary elements in $\mathfrak{S}$ by well-behaved elements. Let $L_{1}(G)$ denote the space of all functions on $G$ which are summable with respect to the Haar measure.

Definitions. Let $\mu(x)$ be any measurable function on $G$ which is real and non-negative and let $\left\{f_{n}(x)\right\}$ be a sequence of functions in $L_{1}(G)$. We shall call it a Dirac $\mu$-sequence if the following conditions hold:

(1) $\int f_{n}(x) d x=1$ and $\lim _{n \rightarrow \infty} \int_{G}\left|f_{n}(x)\right| d x=1$.

(2) For any measurable neighbourhood $V$ of 1 in $G$,

$$
\lim _{n \rightarrow \infty} \int_{G-\nabla}\left|f_{n}(x)\right|(1+\mu(x)) d x=0 .
$$

If $\mu=1$ we call it just a Dirac sequence.

Lemma 25. Suppose there exists a Dirac $|\pi(x)|$-sequence $\left\{f_{n}(x)\right\}$ on $G$ such that $f_{n} \in \mathfrak{B}_{\pi}$. Then the space $W$ of all well-behaved elements is dense in $\mathfrak{W}$. 
Let $\psi$ be an element in $\mathfrak{S}$. Given any $\epsilon>0$ we can find, due to the continuity of the representation $\pi$, an open neighbourhood $V$ of 1 in $G$ such that $|\pi(x) \psi-\psi| \leqq \epsilon|\psi|$ for $x \in V$. Then if $\psi_{n}=T_{f_{n}} \psi$,

$$
\begin{aligned}
\psi_{n}-\psi & =\int_{G} f_{n}(x)(\pi(x) \psi-\psi) d x \\
& =\int_{V} f_{n}(x)(\pi(x) \psi-\psi) d x+\int_{G-V} f_{n}(x)(\pi(x) \psi-\psi) d x .
\end{aligned}
$$

Therefore

$$
\left|\psi_{n}-\psi\right| \leqq \epsilon|\psi| \int_{G}\left|f_{n}(x)\right| d x+|\psi| \int_{G-V}\left|f_{n}(x)\right|(1+|\pi(x)|) d x \leqq 2 \epsilon|\psi|
$$

if $n$ is sufficiently large. Hence $\psi=\lim _{n \rightarrow \infty} \psi_{n}$. But since $\psi_{n} \in W$ (Lemma 24), it follows that $W$ is dense in $\mathfrak{S}$.

We shall now give a method for constructing such a sequence under suitable assumptions regarding $G$.

Suppose $g_{0}$ is the direct sum of two subalgebras $\mathfrak{t}_{0}$ and $\xi_{0}$ and $K$ and $S$ are the analytic subgroups of $G$ corresponding to $\mathfrak{I}_{0}$ and $\varepsilon_{0}$ respectively. Consider the mapping $\Phi:(u, s) \rightarrow u s(u \in K, s \in S)$ of $K \times S$ into $G$. Clearly it is analytic.

Lemma 26. $\Phi$ is everywhere regular $\left(^{8}\right)$ on $K \times S$.

Since the tangent space of a Lie group at any point may be identified under left translation by its Lie algebra (which is the tangent space at 1), it is clear that the tangent space of $K \times S$ at any point is the direct sum of $\mathfrak{f}_{0}$ and $\xi_{0}$ and therefore it may be identified with $g_{0}$. Let $d \Phi$ be the differential of $\Phi$ and let $u_{0} \in K$ and $s_{0} \in S$ be two fixed elements. Then we see without difficulty that $(d \Phi)_{u_{0}, \varepsilon_{0}} U=\operatorname{Ad}\left(s_{0}^{-1}\right) U,(d \Phi)_{u_{0}, \varepsilon_{0}} Y=Y$ for $Y \in \mathfrak{l}_{0}$ and $U \in \mathbb{8}_{0}$. Here $x \rightarrow \operatorname{Ad}(x)$ is the adjoint representation of $G$. Let $s \rightarrow \operatorname{Ad}(s)(s \in S)$ denote the adjoint representation of $S$. Then it follows that

$$
\operatorname{det}\left((d \Phi)_{u_{0}, s_{0}}\right)=\frac{\operatorname{det} \operatorname{Ad}_{s}\left(s_{0}\right)}{\operatorname{det} \operatorname{Ad}\left(s_{0}\right)} \neq 0
$$

and so $\Phi$ is regular at $\left(u_{0}, s_{0}\right)$.

Corollary. If $\Phi$ is a 1-1 mapping of $K \times S$ onto $G$, it is an analytic isomorphism of $K \times S$ with $G$.

For since $\Phi$ is regular, the inverse mapping is analytic.

Lemma 27. Suppose $\Phi$ is a 1-1 mapping of $K \times S$ onto G. Let $d u, d s$, and $d x$ denote the left-invariant Haar measure on $K, S$, and $G$ respectively. Then under suitable normalisation of these measures we have 


$$
d x=\frac{\operatorname{det} \operatorname{Ad}_{S}(s)}{\operatorname{det} \operatorname{Ad}(s)} d u d s \quad(x=u s, u \in K, s \in S) .
$$

This follows immediately from the fact that

$$
\operatorname{det}\left((d \Phi)_{u, s}\right)=\operatorname{det} \operatorname{Ad}_{\boldsymbol{s}}(s) / \operatorname{det} \operatorname{Ad}(s) .
$$

Definition. Let $\$$ be a Lie algebra over a field of characteristic zero. We say that 8 is quasi-nilpotent if it can be written as the direct sum of an abelian subalgebra a and a nilpotent ideal $\mathfrak{n}$. A connected Lie group $S$ is called quasi-nilpotent if its Lie algebra is quasi-nilpotent.

From now on let $S$ denote a simply-connected quasi-nilpotent Lie group and $z_{0}$ its Lie algebra. Let $z_{0}=\mathfrak{a}_{0}+\mathfrak{n}_{0}$ where $\mathfrak{a}_{0}$ is an abelian subalgebra and $\mathfrak{n}_{0}$ a nilpotent ideal in $\Xi_{0}$ and the sum is direct. It is known that there exists a faithful matrix representation of $S$ (see for example [8 (b)]). Therefore we may consider $S$ a linear group and $B_{0}$ a linear Lie algebra. Since every matrix with complex coefficients may also be regarded as a real matrix of twice the degree, we may further assume that all matrices in $S$ and $\xi_{0}$ are real. Let 8 be the Lie algebra over $C$ spanned by $\delta_{0}$. Then is the complexification of $8_{0}$. Let $S_{c}$ be the analytic group of matrices corresponding to 8 . Then $S_{c} \supset S$ and, since 8 is a complex algebra, $S_{c}$ is a complex analytic group. Thus we have imbedded $S$ in a complex analytic group $S_{c}$ whose Lie algebra $\&$ is the complexification of $\boldsymbol{B}_{0}$. We may assume that $S_{c}$ is simply-connected, for otherwise we could replace it by its universal covering group and thus obtain an imbedding of $S$ into a simply-connected complex group. Let $\mathfrak{a}$ and $\mathfrak{n}$ be the subalgebra of spanned by $\mathfrak{a}_{0}$ and $\mathfrak{n}_{0}$ respectively, and $A_{c}, N_{c}, A$, and $N$ the analytic subgroups corresponding to $\mathfrak{a}, \mathfrak{n}, \mathfrak{a}_{0}$, and $\mathfrak{n}_{0}$ respectively. All these groups are simply-connected and $(n, a) \rightarrow n a\left(n \in N_{c}, a \in A_{c}\right)$ is a topological mapping of $N_{c} \times A_{c}$ onto $S_{c}$. Moreover, $n a \in S$ if and only if $n \in N$ and $a \in A$. Since $\mathfrak{a}$ and $\mathfrak{n}$ are complex Euclidean spaces we may regard them as complex analytic manifolds in the obvious way. Then the mappings $H \rightarrow \exp H$ and $X \rightarrow \exp X(H \in \mathfrak{a}, X \in \mathfrak{n})$ define holomorphic isomorphisms of $\mathfrak{a}$ with $A_{c}$ and $\mathfrak{n}$ with $N_{c}$ respectively.

For any $x \in S_{c}$ and $n \in N_{c}$ write $x n=\tau_{x}(n) a$ where $\tau_{x}(n) \in N_{c}$ and $a \in A_{c}$. Then $\tau_{x}$ is a topological mapping of $N_{c}$ onto itself and $\tau_{x^{-1}} y=\left(\tau_{x}\right)^{-1} \tau_{y}\left(x, y \in S_{c}\right)$. Let $\theta$ denote the conjugation of 8 with respect to its real form $\varepsilon_{0}$, so that $\theta\left(X+(-1)^{1 / 2} Y\right)=X-(-1)^{1 / 2} Y\left(X, Y \in B_{0}\right)$. We extend $\theta$ to an automorphism $x \rightarrow \theta x$ of $S_{c}$.

Let $f$ be a complex-valued function on $N_{c}$. We shall say that $f$ is linear (polynomial) if the function $X \rightarrow f(\exp X)(X \in \mathfrak{n})$ is a linear (polynomial) function on $\mathfrak{n}$. Similarly, we call a function $f$ on $N_{c} \times N_{c}$ a polynomial function if $f\left(n, n^{\prime}\right)=\sum_{1 \leqq i \leqq k} f_{i}(n) f_{i}^{\prime}\left(n^{\prime}\right)\left(n, n^{\prime} \in N_{c}\right)$ where $f_{i}, f_{i}^{\prime}(1 \leqq i \leqq k)$ are polynomial functions on $N_{c}$. Let $\left(l_{1}, \cdots, l_{r}\right)$ be a base for linear functions on $N_{c}$. Then every polynomial function $f$ on $N_{c}$ is uniquely expressible as a poly- 
nomial $P\left(l_{1}, \cdots, l_{r}\right)$ in $l_{1}, \cdots, l_{r}$. We define the degree of $f$ to be the degree of $P$. It is then clear that the polynomial functions of degree $\leqq \mu$ form a vector space of finite dimension.

For any function $f$ on $N_{c}$ we denote by $f_{x}\left(x \in S_{c}\right)$ the function $n$ $\rightarrow f\left(\tau_{x^{-1}}(n)\right)\left(n \in N_{c}\right)$. If $f$ is a polynomial function, then so is $f_{x}$. It is clearly sufficient to prove this when $f$ is linear. It is then known that for any $n_{0} \in N_{c}$ the function $n \rightarrow f\left(n_{0} n\right)$ is a polynomial function (see Birkhoff [2]). Now if $x^{-1}=n_{0} a_{0}\left(n_{0} \in N_{c}, a_{0} \in A_{c}\right), f\left(\tau_{x^{-1}}(n)\right)=f\left(n_{0} a_{0} n a_{0}^{-1}\right)=f_{1}\left(a_{0} n a_{0}^{-1}\right)$ where $f_{1}$ is a polynomial function. On the other hand

$$
a_{0}(\exp X) a_{0}^{-1}=\exp \left(\operatorname{Ad}\left(a_{0}\right) X\right)
$$

where $y \rightarrow \operatorname{Ad}(y)$ is the adjoint representation of $S_{c}$. Hence the functions $n \rightarrow l_{i}\left(a_{0} n a_{0}^{-1}\right) \quad(1 \leqq i \leqq r)$ are all linear and this proves that the function $f_{x}: n \rightarrow f_{1}\left(a_{0} n a_{0}^{-1}\right)$ is a polynomial function. Moreover if $l$ is a linear function on $N_{c}$, the function $\left(n_{0}, n\right) \rightarrow l\left(n_{0} n\right)$ is a polynomial function on $N_{c} \times N_{c}$ (see Birkhoff [2]) and so it follows that there exists an integer $\mu$ independent of $n_{0}$ such that every function of the form $n \rightarrow l\left(n_{0} n\right)$ ( $l$ linear) is of degree $\mu$. This means that the vector space $\mathfrak{Q}$ spanned by the functions $\left(l_{i}\right)_{x}(1 \leqq i \leqq r$, $\left.x \in S_{c}\right)$ is finite-dimensional. Since $\left(f_{x}\right)_{y}=f_{y x}$ for any function $f$ on $N_{c}$ $(x, y \in G)$, it follows that $Q_{x} \in \mathfrak{Q}$ for every $Q \in \mathfrak{Q}$.

We may choose the functions $l_{i}, 1 \leqq i \leqq r$, in such a way that they are real on $N$. Then $l_{i}(\theta n)=\overline{l_{i}(n)}\left(n \in N_{c}\right)$ where the bar denotes complex-conjugate. From this it follows that $\left(l_{i}\right)_{\theta x}$ coincides with $\overline{\left(l_{i}\right)_{x}}$ on $N$. Hence for every $Q \in \mathfrak{Q}$ there is a function $Q^{\prime} \in \mathfrak{Q}$ which coincides with $\bar{Q}$ on $N$. This shows that $\mathfrak{Q}$ is spanned by functions which are real on $N$. Moreover $l_{i} \in \mathfrak{Q}$, $1 \leqq i \leqq r$. Let $\left(Q_{1}, \cdots, Q_{m}\right)$ be a base for $\mathfrak{Q}$ such that the functions $Q_{j}$ are real on $N$ and the functions $l_{i}, 1 \leqq i \leqq r$, are included among $\left(Q_{1}, \cdots, Q_{m}\right)$. Then

$$
\left(Q_{i}\right)_{x}=\sum_{j=1}^{m} a_{i j}(x) Q_{j}
$$

and the mapping $x \rightarrow\left(a_{i j}(x)\right)=\alpha(x)$ is a matrix representation of $S_{c}$. If $l$ is a linear function on $N_{c},(n, x) \rightarrow l_{x}(n)$ is clearly a holomorphic function on $N_{c} \times S_{c}$. Therefore the same holds for the functions $(n, x) \rightarrow f_{x}(n)$ where $f$ is a polynomial function on $N_{c}$. Therefore the functions $a_{i j}$ are holomorphic on $S_{c}$. Let $\Re c$ denote the real part of a complex number $c$.

Lemma 28. Let $c_{j}, 1 \leqq j \leqq m$, be given complex numbers and let $x_{i}^{\prime}+c_{i}$ $=\sum_{j=1}^{m} b_{i j}\left(x_{j}+c_{j}\right)\left(b_{i j} \in C, x_{j} \in R\right)$. There exists a constant $\epsilon>0$ such that if $\max _{i, j}\left|b_{i j}-\delta_{i j}\right| \leqq \epsilon$, then

$$
\Re\left(\sum_{i=1}^{m} x_{i}^{\prime 2}\right) \geqq \frac{1}{2} \sum_{i=1}^{m} x_{i}^{2}
$$


for all systems of real numbers $x_{1}, \cdots, x_{m}$ such that $\sum_{i=1}^{m} x_{i}^{2} \geqq 1$.

Put $c_{i j}=b_{i j}-\delta_{i j}, M=\max _{i, j}\left|c_{i j}\right|$, and $c=\max _{j}\left|c_{j}\right|$. Then

$$
x_{i}^{\prime}=x_{i}+\sum_{j} c_{i j}\left(x_{j}+c_{j}\right)
$$

and therefore

$$
\sum_{i} x_{i}^{\prime 2}=\sum_{i} x_{i}^{2}+2 \sum_{i, j} c_{i j} x_{i}\left(x_{j}+c_{j}\right)+\sum_{i}\left(\sum_{j} c_{i j}\left(x_{j}+c_{j}\right)\right)^{2} .
$$

Hence

$$
\left|\sum_{i}\left(x_{i}^{\prime 2}-x_{i}^{2}\right)\right| \leqq m\left(2 M+2 M c+M^{2}(1+c)^{2}\right) \sum_{i} x_{i}^{2}
$$

provided $\sum_{i} x_{i}^{2} \geqq 1$. Hence if $M$ is sufficiently small

$$
\left|\sum_{i}\left(x_{i}^{\prime 2}-x_{i}^{2}\right)\right| \leqq \frac{1}{2} \sum_{i} x_{i}^{2}
$$

and therefore

$$
\Re\left(\sum_{i=1}^{m} x_{i}^{\prime 2}\right) \geqq \frac{1}{2} \sum_{i=1}^{m} x_{i}^{\prime 2} .
$$

Since the functions $a_{i j}(x)$ are continuous and $a_{i j}(1)=\delta_{i j}$ it follows from the above lemma that there exists a neighbourhood $V^{\prime}$ of 1 in $S_{c}$ such that if

$$
u(n a)=\sum_{i=1}^{n}\left(Q_{i}(n)-Q_{i}(1)\right)^{2} \quad\left(n \in N_{c}, a \in A_{c}\right),
$$

then

$$
\Re\left(u\left(x_{0} n a\right)\right) \geqq \frac{1}{2} u(n) \geqq \frac{1}{2} \sum_{i=1}^{r} l_{i}^{2}(n)
$$

for $n \in N, a \in A$, and $x_{0} \in V^{\prime}$ provided $\sum_{i=1}^{r} l_{i}^{2}(n) \geqq 1$.

Let us call a function $\lambda$ on $A_{c}$ linear if the function $H \rightarrow \lambda(\exp H)(H \in \mathfrak{a})$ is linear on $a$. Choose a base $\left(\lambda_{1}, \cdots, \lambda_{s}\right)$ for the space of linear functions on $A_{c}$ such that $\lambda_{i}$ are real on $A$ and let $\lambda_{0}$ denote the constant function 1 . Then if $a, a^{\prime} \in A_{c}, \lambda_{i}\left(a a^{\prime}\right)=\lambda_{i}\left(a^{\prime}\right)+\lambda_{i}(a) \lambda_{0}\left(a^{\prime}\right), 1 \leqq i \leqq s$. Put

$$
v(n a)=\sum_{j=1}^{s} \lambda_{j}^{2}(a) \quad\left(n \in N_{c}, a \in A_{c}\right) .
$$

Then making use of an argument similar to that of Lemma 28, we see that there exists a compact neighbourhood $V$ of 1 in $S_{c}\left(V \subset V^{\prime}\right)$ such that 


$$
\Re\left(v\left(x_{0} n a\right)\right) \geqq \frac{1}{2} v(n a) \geqq \frac{1}{2} \sum_{j=1}^{8} \lambda_{j}^{2}(a)
$$

for $x_{0} \in V, n \in N$, and $a \in A$. Now put $F_{\nu}(x)=e^{-\nu\{u(x)+v(x)\}}\left(x \in S_{c}\right)$ where $\nu$ is a positive real number. Then $F_{\nu}$ is holomorphic on $S_{c}$ and

$$
\left|F_{\nu}\left(x_{0} x\right)\right| \leqq M_{\nu} \exp \left\{-\frac{\nu}{2}\left(\sum_{i=1}^{r} l_{i}^{2}(n)+\sum_{j=1}^{s} \lambda_{j}^{2}(a)\right)\right\}
$$

for $x_{0} \in V$ and $x \in S(x=n a, n \in N, a \in A)$. Here $M_{\nu}$ is the supremum of $e^{\nu / 2}\left|e^{-\nu u\left(x_{0}\right)}\right|$ for $x_{0} \in V$, and $\sum l_{t}^{2}(n) \leqq 1(n \in N)$.

Now let $\pi$ be a representation of $S$ on a Banach space $\mathfrak{W}$. We shall prove that $F_{\nu} \in \mathfrak{B}_{\pi}$. Let us say that a function $f$ on $S$ is of at most exponential growth if there exist real constants $M_{1}, M_{2} \geqq 0$ such that

$$
|f(n a)| \leqq M_{1} \exp \left(M_{2} \max _{i, j}\left(\left|l_{i}(n)\right|,\left|\lambda_{j}(a)\right|\right)\right) \quad(n \in N, a \in A) .
$$

Clearly the product of two such functions is again of the same type. Now consider the function $|\pi(x)|$. Let $M$ denote an upper bound for $|\pi(x)|$ on the compact set consisting of all points $x=n a$ in $S(n \in N, a \in A)$ such that $\max _{i, j}\left\{\left|l_{i}(n)\right|,\left|\lambda_{j}(a)\right|\right\} \leqq 1$. For any $x \in S$ let $q$ denote the least integer such that $q \geqq \max _{i, j}\left\{\left|l_{i}(n)\right|,\left|\lambda_{j}(a)\right|\right\} \quad(x=n a)$. Then we can choose elements $n^{\prime} \in N$ and $a^{\prime} \in A$ such that $n=n^{\prime q}$ and $a=a^{\prime q}$ and

$$
|\pi(x)| \leqq|\pi(n)||\pi(a)| \leqq\left|\pi\left(n^{\prime}\right)\right|^{q}\left|\pi\left(a^{\prime}\right)\right|^{q} \leqq M^{2 q}
$$

since $\left|l_{i}\left(n^{\prime}\right)\right|=(1 / q)\left|l_{i}(n)\right| \leqq 1$ and $\left|\lambda_{j}\left(a^{\prime}\right)\right|=(1 / q)\left|\lambda_{j}(a)\right| \leqq 1$. Since $q \leqq 1$ $+\max _{i, j}\left\{\left|l_{i}(n)\right|, \lambda_{j}(a)\right\}$, we see that $|\pi(x)|$ is at most of exponential growth. Now consider the Haar measure $d x$ on $S$. Making use of Lemma 27 we find that

$$
d x=\operatorname{det}\left(\operatorname{Ad}_{S}\left(a^{-1}\right)\right) d n d a \quad(x=n a)
$$

when $\operatorname{Ad}_{S}$ is the adjoint representation of $S$ and $d n, d a$ are the Haar measures of $N$ and $A$ respectively. Since $N$ is nilpotent and $A$ is abelian,

$$
d n d a=d l_{1} \cdots d l_{r} d \lambda_{1} \cdots d \lambda_{s} .
$$

Moreover, since the function $n a \rightarrow \operatorname{det} \operatorname{Ad}_{S}\left(a^{-1}\right)$ is clearly of at most exponential growth, it follows that

$$
|\pi(x)| d x=\mu(x) d l_{1} \cdots d l_{r} d \lambda_{1} \cdots d \lambda_{s},
$$

where $\mu(x)$ is a function of at most exponential growth. Therefore it is clear that

$$
\int g_{v}(x)|\pi(x)| d x<\infty
$$


where

$$
g_{\nu}(n a)=\exp \left\{-\frac{\nu}{2}\left(\sum_{i=1}^{r} l_{i}^{2}(n)+\sum_{i=1}^{s} \lambda_{i}^{2}(a)\right)\right\} \quad(n \in N, a \in A) .
$$

We have seen above that we can choose an open connected neighbourhood $W$ of 0 in 8 such that

$$
\left|F_{\nu}(\exp (-Z) x)\right| \leqq M_{\nu} g_{\nu}(x) \quad(Z \in W, x \in S)
$$

where $Z \rightarrow \exp Z$ is the exponential mapping of into $S_{c}$. Therefore the function $x \rightarrow F_{\nu}(\exp (-Z) x)$ is summable with respect to the measure $|\pi(x)| d x$ on $S$. Let $L(S, \pi)$ denote the Banach space of functions corresponding to the representation $\pi$, which was introduced in $\S 7$, and let $\psi_{\nu}(Z)$ be the element in $L(S, \pi)$ which is represented by the function $x \rightarrow F_{\nu}(\exp (-Z) x)(Z \in W)$. Then all the conditions of Lemma 23 are fulfilled and therefore $Z \rightarrow \psi_{\nu}(Z)$ is a holomorphic mapping of $W$ into $L(S, \pi)$. Let $\sigma$ denote the representation of $S$ on $L(S, \pi)$ as it was defined in $\S 7$. Then $\sigma(\exp X) \psi_{\nu}=\psi_{\nu}(X)\left(X \in 8_{0}\right)$ where $\psi_{\nu}$ is the element in $L(S, \pi)$ corresponding to $F_{\nu}$. Therefore the mapping $X \rightarrow \sigma(\exp X) \psi_{\nu}\left(X \in \mathbb{8}_{0}\right)$ is analytic at $X=0$ and so $F_{\nu} \in \mathfrak{S}_{\pi}$.

Now consider $J_{\nu}=\int F_{\nu}(x) d x$. If $n \in N$ and $a \in A$, let $n_{\nu}$ and $a_{\nu}$ denote two elements in $N$ and $A$ respectively such that $l_{i}\left(n_{\nu}\right)=\nu^{1 / 2} l_{i}(n), 1 \leqq i \leqq r$, and $\lambda_{j}\left(a_{\nu}\right)=\nu^{1 / 2} \lambda_{j}(a), 1 \leqq j \leqq s$. Moreover if

$$
P=\sum_{(p)} c\left(p_{1}, \cdots, p_{r}\right) l_{1}^{p_{1}} \cdots l_{r}^{p_{r}} \quad\left(c\left(p_{1}, \cdots, p_{r}\right) \in R\right)
$$

is any polynomial function on $N_{c}$, we put $P^{*}(n)=\sum_{(p)}\left|c\left(p_{1}, \cdots, p_{r}\right)\right|$ $\left|l_{1}(n)\right| p_{1} \cdots\left|l_{r}(n)\right| p_{r}$. Then it is clear that $\nu^{1 / 2}|P(n)| \leqq \nu^{1 / 2} P^{*}(n) \leqq P^{*}\left(n_{\nu}\right)$ if $P(1)=0$. Hence if $Q_{i}^{\prime}(n)=Q_{i}(n)-Q_{i}(1)$,

$$
\begin{aligned}
F_{\nu}(n a) & =\exp \left\{-\nu \sum_{i=1}^{m} Q_{i}^{\prime 2}(n)-\nu \sum_{j=1}^{s} \lambda_{j}^{2}(a)\right\} \\
& \geqq \exp \left\{-\sum_{i=1}^{m} Q_{i}^{\prime * 2}\left(n_{\nu}\right)-\sum_{j=1}^{s} \lambda_{j}^{2}\left(a_{\nu}\right)\right\} .
\end{aligned}
$$

On the other hand

$$
J_{\nu}=\int F_{\nu}(n a) \operatorname{det}\left(\exp \operatorname{Ad}_{S}\left(a^{-1}\right)\right) d l_{1} \cdots d l_{r} d \lambda_{1} \cdots d \lambda_{S}
$$

and we can find real numbers $M_{1}, M_{2}>0$ such that

$$
\operatorname{det}\left(\exp \operatorname{Ad}_{S}(a)\right) \leqq M_{1} \exp \left(M_{2} \sum_{i=1}^{s}\left|\lambda_{i}(a)\right|\right)
$$

Hence 


$$
\operatorname{det}\left(\exp \operatorname{Ad}_{s}\left(a^{-1}\right)\right) \geqq M_{1}^{-1} \exp \left(-M_{2} \sum_{i=1}^{8}\left|\lambda_{i}(a)\right|\right)
$$

and

$$
\begin{aligned}
J_{\nu} \geqq M_{1}^{-1} \int \exp & \left\{-\sum_{i=1}^{m} Q_{i}^{\prime * 2}\left(n_{\nu}\right)-\sum_{j=1}^{s} \lambda_{j}^{2}\left(a_{v}\right)\right. \\
& \left.-M_{2} \sum_{j=1}^{s}\left|\lambda_{j}\left(a_{v}\right)\right|\right\} d l_{1} \cdots d l_{r} d \lambda_{1} \cdots d \lambda_{s}=\frac{c}{\nu^{(r+s) / 2}}>0,
\end{aligned}
$$

where $c$ is the value of the right-hand side when $\nu=1$. On the other hand, let $V_{\delta}$ be the neighbourhood of 1 in $S$ consisting of all points na $\in S$ such that $\left|l_{i}(n)\right|<\delta,\left|\lambda_{i}(a)\right|<\delta(\delta>0)$. Then let us consider the integral

$$
J_{\nu}(\delta)=\int_{S-\nabla_{\delta}} F_{\nu}(x)(1+|\pi(x)|) d x .
$$

We can find positive constants $M_{3}$ and $M_{4}$ such that

$$
(1+|\pi(n a)|) \operatorname{det}\left(\exp \operatorname{Ad}_{S}\left(a^{-1}\right)\right) \leqq M_{3} \exp M_{4}\left(\sum_{i=1}^{r}\left|l_{i}(n)\right|+\sum_{j=1}^{\dot{r}}\left|\lambda_{j}(a)\right|\right) .
$$

Put

$$
P_{1}(n, a)=\sum_{i=1}^{r} l_{i}^{2}(n)+\sum_{j=1}^{s} \lambda_{j}^{2}(a) \text { and } P_{2}(n, a)=\sum_{i=1}^{r}\left|l_{i}(n)\right|+\sum_{j=1}^{s}\left|\lambda_{j}(a)\right| \text {. }
$$

Then $\nu P_{1}(n, a)=P_{1}\left(n_{\nu}, a_{\nu}\right)$ and $\nu^{1 / 2} P_{2}(n, a)=P_{2}\left(n_{\nu}, a_{\nu}\right)$ and therefore

$$
\begin{aligned}
J_{\nu}(\delta) \leqq & M_{3} \int_{S-V_{\delta}} \exp \left\{-\nu P_{1}(n, a)+\nu^{1 / 2} M_{4} P_{2}(n, a)\right\} d l_{1} \cdots d l_{r} d \lambda_{1} \cdots d \lambda_{s} \\
\leqq & M_{3} e^{-\nu(r+s) \delta^{2} / 2} \int_{S-V_{\delta}} \exp \left\{-\frac{\nu}{2} P_{1}(n, a)\right. \\
& \left.\quad+\nu^{1 / 2} M_{4} P_{2}(n, a)\right\} d l_{1} \cdots d l_{r} d \lambda_{1} \cdots d \lambda_{s}
\end{aligned}
$$

where

$$
c^{\prime}=M_{3} \int_{S-V_{3}} \exp \left\{-\frac{1}{2} P_{1}(n, a)+M_{4} P_{2}(n, a)\right\} d l_{1} \cdots d l_{r} d \lambda_{1} \cdots d \lambda_{2}<\infty .
$$

Therefore 


$$
\frac{J_{\nu}(\delta)}{J_{\nu}} \leqq \frac{c^{\prime}}{c} e^{-\nu(r+s) \delta^{2} / 2 \cdot \nu^{\nu+\rho}}
$$

and so

$$
\lim _{\nu \rightarrow \infty} \frac{J_{\nu}(\delta)}{J_{\nu}}=0 .
$$

Hence if we put $f_{\nu}=F_{\nu} / J_{\nu}$, the sequence $\left\{f_{1}, f_{2}, \ldots\right\}$ is a $|\pi(x)|$-Dirac-sequence of functions in $\mathfrak{W}_{\pi}$. Therefore from Lemma 25 the space of wellbehaved elements is dense in $\mathfrak{S}$. We can now prove the following theorem:

THEOREM 3. Suppose a connected Lie group $G$ has two analytic subgroups $K$ and $S$ such that the following two conditions are fulfilled: (a) $K$ is compact and $S$ is quasi-nilpotent; (b) every element in $G$ can be written uniquely in the form us $(u \in K, s \in S)$. Let $\pi$ be a representation of $G$ on a Banach space $\mathfrak{S}$ and let $\mathfrak{S}_{0}$ denote the set of all well-behaved elements $\psi$ in $\mathfrak{S}$ such that the linear space spanned by the elements $\pi(u) \psi(u \in K)$ is of finite dimensions. Then $\mathfrak{S}_{0}$ is dense in $\mathfrak{S}$.

The restriction of $\pi$ on $S$ defines a representation $\rho$ of $S$ on $\mathfrak{S}$. Since $\rho$ may also be regarded as a representation of the simply-connected covering group of $S$, it follows from the result proved above that the set of elements which are well-behaved under $\rho$ is dense in $\mathfrak{S}$. Hence given any $\psi_{0} \in \mathfrak{S}$ and $\epsilon>0$ we can find an element $\psi$ which is well-behaved under $\rho$ and such that $\left|\psi_{0}-\psi\right| \leqq \epsilon$. Choose a neighborhood $U$ of 1 in $K$ such that $|\pi(u) \psi-\psi| \leqq \epsilon$ for $u \in U$ and let $d u$ denote the element of Haar measure on $K$ such that $\int_{K} d u=1$. Select a continuous, real, non-negative function $f$ on $K$ such that $f=0$ outside $U$ and $\int_{K} f d u=1$. Then if $\psi_{1}=\int_{K} f(u) \pi(u) \psi d u$,

$$
\left|\psi_{1}-\psi\right| \leqq \int_{K} f(u)|\pi(u) \psi-\psi| d u \leqq \epsilon .
$$

Let $\Re$ be the set of all finite linear combinations (with complex coefficients) of the coefficients of finite-dimensional matrix representations of $K$. Then for any $\eta>0$ we can find a function $\omega \in \Re$ such that $|f(u)-\omega(u)| \leqq \eta(u \in K)$. Hence

$$
\left|\int_{K}(f(u)-\omega(u)) \pi(u) \psi d u\right| \leqq M \eta|\psi| \leqq M \eta\left(\left|\psi_{0}\right|+\epsilon\right)
$$

where $M$ is an upper bound for $|\pi(u)|$ on the compact set $K$. Therefore if

$$
\psi_{2}=\int_{K} \omega(u) \pi(u) \psi d u,\left|\psi_{0}-\psi_{2}\right| \leqq 2 \epsilon+M \eta\left(\left|\psi_{0}\right|+\epsilon\right) \leqq 3 \epsilon
$$

if $\eta$ is sufficiently small. Since $\omega \in \Re$ we see easily that the elements 
$\pi(u) \psi_{2}(u \in K)$ span a finite-dimensional subspace of $\mathfrak{W}$. Therefore in order to prove the theorem it is sufficient to show that the element $\psi_{2}$ is wellbehaved under $\pi(G)$. This follows from the lemma given below.

LEMMA 29. Let $\omega$ be an analytic function on $K$ and $\psi$ an element in $\mathfrak{S}$ which is well-behaved under $\rho$. Then $\int_{K} \omega(u) \pi(u) \psi d u$ is well-behaved under $\pi(G)$.

For any $x \in G$ and $u \in K$ put $x u=u_{x} s(x, u)$ where $u_{x} \in K$ and $s(x, u) \in K$. Then we know (see the corollary to Lemma 26) that $(x, u) \rightarrow u_{x}$ and $(x, u)$ $\rightarrow s(x, u)$ are analytic mappings of $G \times K$ into $K$ and $S$ respectively. Now put $\psi_{0}=\int_{K} \omega(u) \pi(u) \psi d u$. Then

$$
\pi(x) \psi_{0}=\int_{K} \omega(u) \pi(x u) \psi d u .
$$

But for $x$ fixed, the mapping $u \rightarrow u_{x}(u \in K)$ is an analytic isomorphism of the manifold structure of $K$ with itself. Since $G$ is connected, this isomorphism is orientation preserving. From this we conclude that

$$
d u_{x}=D(x, u) d u
$$

where $D(x, u)$ is an analytic function of $G \times K$ which is everywhere positive. Hence

$$
\begin{aligned}
\pi(x) \psi_{0} & =\int_{K} \omega\left(u_{x^{-1}}\right) \pi\left(x u_{x^{-1}}\right) \psi d u_{x^{-1}} \\
& =\int_{K} \omega\left(u_{x^{-1}}\right) D\left(x^{-1}, u\right) \pi(u) \pi\left(\left(s\left(x^{-1}, u\right)\right)^{-1}\right) \psi d u .
\end{aligned}
$$

On the other hand, since $\psi$ is well-behaved under $\rho$, it follows from Lemmas 16 and 17 that the mapping is an analytic mapping of $G \times K$ into $\mathfrak{S}$. Let $t_{1}, \cdots, t_{n}$ be a coordinate system in $G$ at 1 such that $t_{j}(1)=0(1 \leqq j \leqq n)$. Then for each $u_{0} \in K$ we can find an open connected neighbourhood $V_{u_{0}}$ of $u_{0}$ in $K$ and a coordinate system $q_{1}, \cdots, q_{k}$ in $K$ valid on $V_{u_{0}}$ with the following properties: (1) $q_{i}\left(u_{0}\right)=0,1 \leqq i \leqq k$; (2) there exists a power series $P_{u_{0}}(q, t)$ in $\left(q_{1}, \cdots, q_{k}\right)$ and $\left(t_{1}, \cdots, t_{n}\right)$ with coefficients in $\mathfrak{S}$ and an open neighbourhood $W_{u_{0}}$ of 1 in $G$ such that the coordinates $\left(t_{1}, \cdots, t_{n}\right)$ are valid on $W_{u_{0}}$ and the power series converges to $\psi(x, u)$ on $W_{u_{0}} \times V_{u_{0}}$. Suppose

$$
P_{u_{0}}(q, t)=\sum_{(a),(e)} \psi_{u_{0}}(a, e) q_{1}^{a_{1}} \cdots q_{k}^{a_{k} t_{1}^{e_{1}}} \cdots t_{n}^{\boldsymbol{e}_{n}} \quad\left(\psi_{u_{0}}(a, e) \in \mathfrak{W}\right)
$$

and put

$$
\psi_{u_{0}}(a, e)=\sum_{(a)} \psi_{u_{0}}(a, e) q_{1}^{a_{1}}(u) \cdots q_{k}^{a_{k}}(u) \quad\left(u \in V_{u_{0}}\right)
$$

Then the mapping $u \rightarrow \psi_{u_{0}}(u, e)$ is an analytic mapping of $V_{u_{0}}$ into $\mathfrak{S}$. Choose 
a number $r\left(u_{0}\right)>0$ such that the series $P_{u_{0}}(q, t)$ converges if $\max _{i, j}\left(\left|q_{j}\right|,\left|t_{i}\right|\right)$ $\leqq r\left(u_{0}\right)$. Let $U_{u_{0}}$ be a neighbourhood of $u_{\theta}$ (in $K$ ) such that $U_{u_{0}} \subset V_{u_{0}}$ and $\max$, $\left|q_{j}(u)\right| \leqq r\left(u_{0}\right)\left(u \in U_{u_{0}}\right)$. Similarly we may suppose that $\max _{i}\left|t_{i}(x)\right| \leqq r\left(u_{0}\right)$ for $x \in W_{u_{0}}$. Since $K$ is compact, there exists a finite set of points $u_{1}, \cdots, u_{p}$ such that $K=U_{u_{1}} \cup U_{u_{2}} \cup \cdots \cup U_{u_{p}}$. Put $W=W_{u_{1}} \cap W_{u_{2}} \cap \cdots \cap W_{u_{p}}$ and $r=\min \left\{r\left(u_{1}\right), \cdots, r\left(u_{p}\right)\right\}$. Then if $u \in U_{u_{i}} \cap U_{u_{j}}(1 \leqq i, j \leqq p)$ and $x \in W$,

$$
\psi(x, u)=\sum_{(e)} \psi_{u_{i}}(u, e) t_{1}^{e_{1}}(x) \cdots t_{n}^{e_{n}}(x)=\sum_{(e)} \psi_{u_{j}}(u, e) t_{1}^{e_{1}}(x) \cdots t_{n}^{e_{n}}(x)
$$

and therefore $\psi_{u_{i}}(u, e)=\psi_{u_{j}}(u, e)$. Thus for each $(e)$ there exists a mapping $u \rightarrow \psi(u, e)$ of $K$ into $\mathfrak{S}$ which coincides on $U_{u_{i}}$ with $u \rightarrow \psi_{u_{i}}(u, e)$. This mapping is clearly analytic and

$$
\psi(x, u)=\sum_{(e)} \psi(u, e) t_{1}^{e_{1}}(x) \cdots t_{n}^{e_{n}}(x) \quad(u \in K, x \in W) .
$$

Now if $u \in U_{u_{i}}$ and $x \in W$,

$$
\sum_{(e)}\left|\psi(u, e) t_{1}^{e_{1}}(x) \cdots t_{n}^{e_{n}}(x)\right| \leqq \sum_{(e)}\left|\psi_{u_{i}}(u, e)\right|\left(r\left(u_{i}\right)\right)^{e_{1}+\cdots+e_{n}}<\infty .
$$

Moreover since $K$ is compact, we can find a real number $M$ such that $|\pi(u)|$ $\leqq M$ for all $u \in K$. Then

$$
\sum_{(e)}\left|\pi(u) \psi(u, e) t_{1}^{e_{1}}(x) \cdots t_{n}^{e_{n}}(x)\right| \leqq M \sum_{(e)}\left|\psi(u, e) t_{1}^{e_{1}}(x) \cdots t_{n}^{e_{n}}(x)\right|
$$

and therefore the series

$$
\sum_{(e)} \pi(u) \psi(u, e) t_{1}^{\epsilon_{1}}(x) \cdots t_{n}^{\epsilon_{n}}(x)
$$

converges uniformly to $\pi(u) \psi(x, u)$ on $K \times W$. Therefore

$$
\begin{aligned}
\pi(x) \psi_{0} & =\int_{K} \pi(u) \psi(x, u) d u \\
& =\sum_{(e)}\left(\int_{K} \pi(u) \psi(u, e) d u\right) t_{1}^{e_{1}}(x) \cdots t_{n}^{e_{n}}(x) \quad(x \in W) .
\end{aligned}
$$

This proves that the mapping $x \rightarrow \pi(x) \psi_{0}$ is analytic at 1 and so $\psi_{0}$ is wellbehaved under $\pi$.

\section{Part III. Representations of a Semisimple Lie group}

9. Permissible representations. We shall now apply the results of Part II to representations of a connected semisimple Lie group $G$ on a Banach space $\mathfrak{S}$. Since every such representation may also be regarded as a representation of the simply-connected covering group of $G$, we may assume that $G$ itself 
is simple-connected. Let $\mathfrak{g}_{0}$ be its Lie algebra. We define $\mathfrak{l}_{0}, \mathfrak{h}_{\mathfrak{p}_{0}}$, and $\mathfrak{n}_{0}$ as in Part I, $\$ 2$. Let $K, A_{+}$, and $N$ be the analytic subgroups of $G$ corresponding to $\mathfrak{g}_{0}, \mathfrak{h}_{p_{0}}$, and $\mathfrak{n}_{0}$ respectively. They are all simply-connected. Moreover, $S=A_{+} N$ is a quasi-nilpotent group, and Iwasawa [9] has shown that the mapping $(u, s) \rightarrow u s(u \in K, s \in S)$ is a topological mapping of $K \times S$ onto $G$. However, in general $K$ is not compact and therefore Theorem 3 is not applicable immediately. Therefore we shall now give a method for extending the results of this theorem to the present case.

We know that $\mathfrak{f}_{0}$ is reductive (Lemma 3 ). Let $\mathfrak{c}_{0}$ be the center of $\mathfrak{f}_{0}$ and $D$ the analytic subgroup of $G$ corresponding to $\mathfrak{c}_{0}$. Then $K$ is the direct product of its commutator group $K^{\prime}$ and $D$. Moreover if $Z$ is the center of $G$, then $K / D \cap Z$ is compact $\left({ }^{9}\right)$. We shall say that a representation $\pi$ of $G$ on $\mathfrak{S}$ is permissible if $\pi(z)$ is a scalar multiple of the unit operator for $z \in D \cap Z$. Assuming that $\pi$ is permissible, we can choose a complex-valued linear function $\mu$ on $\mathfrak{c}_{0}$ such that $\pi(\exp \Gamma)=e^{\mu(\Gamma)} \pi(1)$ whenever $\exp \Gamma \in D \cap Z\left(\Gamma \in \mathfrak{c}_{0}\right)$. Let $u \rightarrow u^{*}$ denote the natural mapping of $K$ on $K^{*}=K / D \cap Z$. Then it is easy to verify that $e^{-\mu(\Gamma)} \pi(u \exp \Gamma)\left(u \in K^{\prime}, \Gamma \in \mathfrak{c}_{0}\right)$ depends only on $(u \exp \Gamma)^{*}$ and so, if we denote it by $\pi^{*}\left((u \exp \Gamma)^{*}\right)$, the mapping $u^{*} \rightarrow \pi^{*}\left(u^{*}\right)$ is a representation of $K^{*}$ on $\mathfrak{S}$. Let $\psi_{0}$ be a given element in $\mathfrak{S}$ and $\epsilon$ a positive number. Since $S$ is quasi-nilpotent, we can find an element $\psi \in \mathfrak{S}$ which is well-behaved under $\pi(S)$ and such that $\left|\psi-\psi_{0}\right| \leqq \epsilon$. Moreover, since $K^{*}$ is compact, we can choose a finite linear combination $\omega$ of the coefficients of a finite-dimensional matrix representation of $K^{*}$ such that

$$
\left|\int_{K^{*}} \omega\left(u^{*}\right) \pi^{*}\left(u^{*}\right) \psi d u^{*}-\psi\right| \leqq \epsilon .
$$

and therefore

$$
\left|\int_{K^{*}} \omega\left(u^{*}\right) \pi^{*}\left(u^{*}\right) \psi d u^{*}-\psi_{0}\right| \leqq 2 \epsilon .
$$

(Here $d u^{*}$ is the element of the Haar measure on $K^{*}$ normalised in such a way that $\int_{K^{*}} d u^{*}=1$.) We now claim that $\phi=\int_{K^{*}} \omega\left(u^{*}\right) \pi^{*}\left(u^{*}\right) \psi d u^{*}$ is wellbehaved under $\pi(G)$. Consider the element

$$
\pi(x) \phi=\int_{K^{*}} \omega\left(u^{*}\right) \pi(x) \pi^{*}\left(u^{*}\right) \psi d u^{*} \quad(x \in G) .
$$

For any $u \in K$ let $\Gamma(u)$ denote the unique element in $c_{0}$ such that $u$ $=(\exp \Gamma(u)) v\left(v \in K^{\prime}\right)$. Moreover let $x u=u_{x} s(x, u)\left(x \in G, u_{x} \in K, s(x, u) \in S\right)$. Then it is easy to verify that for a fixed $x$ the elements $\left(u_{x}\right)^{*}, \Gamma\left(u_{x}\right)-\Gamma(u)$, and $s(x, u)$ depend only on $u^{*}$. Hence we may write them as $u_{x}^{*}, \Gamma\left(x, u^{*}\right)$, and

( $\left.{ }^{g}\right)$ This follows from the fact that the image of $K$ into the adjoint group of $G$ is compact (see for example Mostow [13]). 
$s\left(x, u^{*}\right)$ respectively. It is clear that the mappings $\left(x, u^{*}\right) \rightarrow u_{x}^{*},\left(x, u^{*}\right)$ $\rightarrow \Gamma\left(x, u^{*}\right),\left(x, u^{*}\right) \rightarrow s(x, u)$ are all analytic and

$$
\begin{aligned}
e^{-\mu(\Gamma(u))} \pi(x) \pi(u) & =e^{-\mu(\Gamma(u))} \pi\left(u_{x}\right) \pi(s(x, u)) \\
& =e^{\mu\left(\Gamma\left(x, u^{*}\right)\right)} \pi^{*}\left(u_{x}^{*}\right) \pi\left(s\left(x, u^{*}\right)\right) .
\end{aligned}
$$

Therefore

$$
\pi(x) \phi=\int_{K^{*}} \omega\left(u^{*}\right) e^{\mu\left(\Gamma\left(x, u^{*}\right)\right)} \pi^{*}\left(u_{x}^{*}\right) \pi\left(s\left(x, u^{*}\right)\right) \psi d u^{*}
$$

and we conclude exactly as in the proof of Lemma 29 that

$$
\pi(x) \phi=\int_{K^{*}} \pi^{*}\left(u^{*}\right) \psi\left(x, u^{*}\right) d u^{*}
$$

where $\left(x, u^{*}\right) \rightarrow \psi\left(x, u^{*}\right)$ is an analytic mapping of $G \times K^{*}$ into $\mathfrak{G}$. Since $K^{*}$ is compact we can now use the same argument as in the proof of Lemma 29 and show that the mapping $x \rightarrow \pi(x) \phi$ is analytic at $x=1$. Therefore $\phi$ is wellbehaved under $\pi$.

Let $\Omega$ be the set of all equivalence classes of finite-dimensional irreducible representations of $K$. For any $\mathfrak{D} \in \Omega$ we denote by $\mathfrak{S}_{\mathfrak{D}}$ the set of all elements $\psi \in \mathfrak{S}$ with the following property: there exists a finite-dimensional linear space $U$ containing $\psi$ which is invariant and semisimple under $\pi(K)$ and is such that the representation of $K$ induced on every simple subspace of $U$ lies in $\mathfrak{D}$. We shall say that an element $\psi \in \mathfrak{S}$ transforms under $K$ (or $\pi(K)$ ) according to $\mathfrak{D}$ if $\psi \in \mathfrak{S}_{\mathfrak{D}}$. Let $W$ be the space of all elements in $\mathfrak{D}$ which are well-behaved under $\pi(G)$. We have seen above that $W \cap\left(\sum \mathbb{D} \in \Omega \mathfrak{D}_{\mathfrak{D}}\right)$ is dense in $\mathfrak{S}$. But since $W$ is stable under $\pi(G)$, it follows that $W \cap\left(\sum \mathscr{D} \in \Omega \mathfrak{D} \mathbb{D}\right)$ $=\sum \sum_{\mathbb{Q}} W_{\mathbb{D}}$ where $W_{\mathbb{D}}=W \cap \Phi_{\mathbb{D}}$ (see Lemma 6). Thus we have the following theorem:

THEOREM 4. Let $\pi$ be a permissible representation of $G$ on $\mathfrak{S}$ and $W$ the set of all well-behaved elements in $\mathfrak{S}$. Then if $W_{\mathbb{D}}=W \cap \mathfrak{D}_{\mathfrak{D}}(\mathfrak{D} \in \Omega)$, the space $\sum_{\mathbb{D} \in \Omega} W_{D}$ is dense in $\mathfrak{S}$.

For any linear subspace $V$ of $\mathfrak{S}$ which is invariant under $\pi(K)$ put $V_{\mathfrak{D}}=V \cap \mathfrak{S}_{\mathbb{D}}$. Then we shall prove the following lemma:

Lemma 30. Let $\pi$ be a permissible representation of $G$ on $\mathfrak{S}$ and $V$ a subspace of $\mathfrak{S}$ invariant under $\pi(K)$. Then if $\sum_{\mathbb{D} \in \Omega} V_{\mathfrak{D}}$ is dense in $\mathfrak{S}, \mathfrak{W}_{\mathfrak{D}}=\mathrm{Cl}\left(V_{\mathfrak{D}}\right)\left({ }^{10}\right)$.

We keep to the notation introduced above. For any $\mathfrak{D} \in \Omega$ let $\chi D$ denote the character of the class $\mathfrak{D}$. Suppose $\mathfrak{E}_{\mathfrak{D}} \neq\{0\}$. Then it is easily seen that $\chi \mathfrak{D}(u) e^{-\mu(\Gamma(u))}(u \in K)$ depends only on $u^{*}$. We may therefore denote it by

(10) $\mathrm{Cl}$ denotes closure in $\mathfrak{S}$. 
$\chi_{\mathfrak{D}}^{*}\left(u^{*}\right)$. Let $E_{\mathfrak{D}}=d(\mathfrak{D}) \int_{K^{*}} \overline{\chi_{\mathfrak{D}}^{*}\left(u^{*}\right)} \pi^{*}\left(u^{*}\right) d u^{*}$ where $d(\mathfrak{D})$ is the degree in $\mathfrak{D}$ and the bar denotes complex conjugate. $E_{D}$ is a bounded linear operator such that $E_{\mathscr{D}}^{2}=E_{\mathfrak{D}}$ and $\mathfrak{S}_{\mathfrak{D}}=E_{\mathfrak{D}} \mathfrak{S}$. Hence $\mathfrak{S}_{\mathfrak{D}}$ as closed in $\mathfrak{S}$. Moreover $V_{0}$ $=\sum_{\mathscr{D} \in \Omega} V_{\mathbb{D}}$ is stable under $\pi(K)$ and the linear space spanned by $\pi(u) \psi(u \in K)$ for any $\psi \in V_{0}$ is of finite dimension. Hence $V_{0}$ is stable under $E_{\mathfrak{D}}$. Now let $\psi \in \mathfrak{S D}_{\mathfrak{D}}$. We can choose a sequence $\psi_{n} \in V_{0}$ such that $\psi=\lim _{n \rightarrow \infty} \psi_{n}$. Then $\psi=E_{\triangleright} \psi=\lim _{n \rightarrow \infty} E_{D} \psi_{n}$. But $E_{\triangleright} \psi_{n} \in V_{0} \cap E_{D} \mathfrak{C} \subset V_{D}$. Therefore $V_{D}$ is dense in $\mathfrak{S}_{\mathfrak{D}}$ and so $\mathfrak{S}_{\mathfrak{D}}=\mathrm{Cl}\left(V_{\mathfrak{D}}\right)$.

Notice that the operator $E_{D}$ is bounded and $E_{D} \psi=\psi$ or 0 according as $\psi \in \mathfrak{S}_{\mathfrak{D}}$ or $\psi \in \mathfrak{S}_{\mathfrak{D}},\left(\mathfrak{D}^{\prime} \neq \mathfrak{D}\right)$. (We define $E_{\mathfrak{D}}$ to be zero in case $\mathfrak{S}_{\mathfrak{D}}=\{0\}$.) Since $\sum_{\mathbb{D}^{\prime} \in \Omega} \mathfrak{S}_{\mathfrak{D}}$, is dense in $\mathfrak{S}, E_{\mathbb{D}}$ is uniquely characterised by these properties. We shall call $E_{\mathbb{D}}$ the canonical projection of $\mathfrak{S}$ on $\mathfrak{S}_{\mathfrak{D}}$. It is clear that $E_{\mathfrak{D}} E_{\mathfrak{D}^{\prime}}=E_{\mathfrak{D}}$ or 0 according as $\mathfrak{D}^{\prime}=\mathfrak{D}$ or $\mathfrak{D}^{\prime} \neq \mathfrak{D}\left(\mathfrak{D}, \mathfrak{D}^{\prime} \in \Omega\right)$.

Let $\pi$ be a representation of $G$ on $\mathfrak{S}$ and $U$ a linear subspace of $\mathfrak{S}$. We shall say that $U$ is differentiable if there exists a representation $\pi_{U}$ of $g_{0}$ on $U$ sưch that $\pi_{U}(X) \psi=\lim _{t \rightarrow 0}(1 / t)(\pi(\exp t X) \psi-\psi)\left(X \in \mathfrak{g}_{0}, \psi \in U\right)$. It is clear that the sum of two differentiable spaces is again differentiable. Hence there exists a largest differentiable space, namely the union of all differentiable subspaces of $\mathfrak{S}$. We say that an element $\psi \in \mathfrak{S}$ is differentiable (under $\pi$ ) if it lies in this union. It is obvious that the Gårding subspace and the space of well-behaved elements are both differentiable. We now state without proof the following lemma.

LeMma 31. Let $\pi$ be a permissible representation of $G$ on $\mathfrak{S}$ and let $E_{\mathfrak{D}}$ denote the projection of $\mathfrak{S}$ on $\mathfrak{S E D}_{\mathbb{D}}$. Then if $\psi$ is a differentiable element in $\mathfrak{S}$, the series $\sum_{\mathbb{D} \in \Omega} E_{\triangleright} \psi$ converges to $\psi$.

We shall not make use of this result anywhere in this paper except in the proof of Theorem 9. A proof of this lemma will be given in a subsequent paper.

10. Quasi-simple representations. Let $\pi$ be a representation of $G$ on $\mathfrak{S}$ and $V$ the Gårding subspace of $\mathfrak{S}$. We denote by $\mathfrak{g}$ the complexification of $\mathfrak{g}_{0}$, by $\mathfrak{B}$ the universal enveloping algebra of $\mathfrak{g}$, by $\mathbb{Z}$ the center of $\mathfrak{B}$, and by $\pi_{V}$ the Gårding representation of $\mathfrak{B}$ on $V$.

Definition. The representation $\pi$ is called quasi-simple if (1) $\pi$ is permissible and (2) there exists a homomorphism $\chi$ of 3 into $C$ such that $\pi_{V}(z) \psi$ $=\chi(z) \psi$ for all $z \in 3$ and $\psi \in V . \chi$ is then called the infinitesimal character of $\pi$.

Let $U$ be the space of all elements in $\mathfrak{S}$ which are differentiable under a representation $\pi$. We denote by $\pi_{U}$ the representation of $\mathscr{B}$ on $U$ such that

$$
\pi_{U}(X) \psi=\lim _{t \rightarrow 0} \frac{1}{t}\{\pi(\exp t X) \psi-\psi\} \quad\left(X \in g_{0}, \psi \in U\right) .
$$

Lemma 32. Let $U_{0}$ be a linear subspace of $U$ and $\chi$ a homomorphism of 3 into $C$ such that $\pi_{U}(z) \psi=\chi(z) \psi$ for all $z \in B$ and $\psi \in U_{0}$. Then if $U_{0}$ is dense in 
$\mathfrak{S}, \pi_{U}(z) \psi=\chi(z) \psi$ for all $z \in \mathfrak{Z}$ and $\psi \in U$.

If we put $U_{0}=V$ in the above lemma we get the following corollary.

COROLLARY. Let $\pi$ be a quasi-simple representation of $G$ on $\mathfrak{S}$ and $\pi_{W}$ the corresponding representation of $B$ on the space $W$ of all well-behaved elements. Then $\pi_{W}(z) \psi=\chi(z) \psi(z \in Z, \psi \in W)$ where $\chi$ is the infinitesimal character of $\pi$.

In order to prove the above lemma, we first establish a simple result which was pointed out to me by Mautner. Let $\widetilde{\mathfrak{F}}$ be the space of all bounded linear functions $\alpha$ on $\mathfrak{S}$ taken with the weak topology. If $\widetilde{\psi} \in \widetilde{\mathfrak{F}}$ and $\psi \in \mathfrak{S}$ we denote by $(\tilde{\psi}, \psi)$ the value of the linear function $\tilde{\psi}$ at $\psi$. Let $A$ be a bounded linear operator on $\mathfrak{S}$. Then the mapping $\tilde{A}: \tilde{\psi} \rightarrow \tilde{A} \tilde{\psi}(\tilde{\psi} \in \tilde{\mathfrak{F}})$ defined by the condition $(\tilde{A} \tilde{\psi}, \psi)=(\tilde{\psi}, A \psi)(\psi \in \mathfrak{S})$ is a continuous linear transformation on $\tilde{\mathfrak{S}}$. $\tilde{A}$ is called the adjoint of $A$. Let $M$ be a linear subspace of $\mathfrak{S}$ and $\mathscr{B}$ a linear transformation of $M$ into $\mathfrak{S}$. We put $|B|_{M}=\sup _{|\psi| \leqq 1}|B \psi|(\psi \in M)$.

Lemma 33. Let $M$ and $N$ be linear subspaces of $\mathfrak{S}$ such that $M \supset N$ and $N$ is dense in $\mathfrak{S}$. Let $A$ and $B$ be linear transformations in $M$ and $N$ respectively such that $A$ coincides with $B$ on $N$. Let $\tilde{M}$ be a dense linear subspace of $\widetilde{\mathfrak{W}}$. Suppose there exists a linear transformation $\tilde{A}$ in $\tilde{M}$ such that $(\tilde{A} \tilde{\phi}, \psi)$ $=(\tilde{\phi}, A \psi)$ for all $\tilde{\phi} \in \tilde{M}$ and $\psi \in M$. Then $|A|_{M}=|B|_{N}$.

It is sufficient to prove that $|A|_{M} \leqq|B|_{N}$ and so we may assume that $\left|B_{N}\right|<\infty$. Now

$$
|(\tilde{A} \tilde{\phi}, \psi)|=|(\tilde{\phi}, A \psi)|=|(\tilde{\phi}, B \psi)| \leqq|B|_{N}|\tilde{\phi}||\psi|
$$

if $\tilde{\phi} \in \tilde{M}, \psi \in N$. Since $N$ is dense in $\mathfrak{E}$ we get by continuity

$$
|(\tilde{A} \tilde{\phi}, \psi)| \leqq|B|_{N}|\tilde{\phi}||\psi| \quad(\tilde{\phi} \in \tilde{M}) .
$$

So in particular if $\psi \in M$

$$
|(\tilde{\phi}, A \psi)|=|(\tilde{A} \tilde{\phi}, \psi)| \leqq|B|_{N}|\tilde{\phi}||\psi| \quad(\tilde{\phi} \in \tilde{M}) .
$$

Since $\tilde{M}$ is dense in $\widetilde{\mathfrak{G}}$ we conclude that

$$
|(\tilde{\phi}, A \psi)| \leqq|B|_{N}|\tilde{\phi}||\psi| \quad(\tilde{\phi} \in \tilde{\mathfrak{F}}, \psi \in M) .
$$

But from a theorem of Banach $[1$, p. 55], we can choose $\tilde{\phi}$ so that $(\tilde{\phi}, A \psi)$ $=|A \psi|$ and $|\tilde{\phi}|=1$. Hence $|A \psi| \leqq|B|_{N}|\psi|$. This proves that $|A|_{M} \leqq|B|_{N}$.

Now we come to the proof of Lemma 32. For any $f \in C_{c}^{\infty}(G)$ let $A_{f}$ denote the operator $\int f\left(x^{-1}\right) \pi(x) d x$ and $\tilde{A}_{f}$ its adjoint. Let $\tilde{V}$ be the subspace of $\tilde{\mathfrak{E}}$ spanned by the elements of the form $\tilde{A}_{f} \tilde{\psi}\left(f \in C_{c}^{\infty}(G), \tilde{\psi} \in \tilde{\mathfrak{E}}\right)$. It is well known that there exists a sequence $\left\{f_{n}\right\}$ of functions in $C_{c}^{\infty}(G)$ with the following properties: (1) $\int f_{n} d x=1$ and $\lim _{n \rightarrow \infty} \int\left|f_{n}\right| d x=1$; (2) all but a finite number of the functions $f_{n}$ are zero outside any given neighbourhood of 1 . From this it follows immediately that $\lim _{n \rightarrow \infty} A_{f_{n}} \psi=\psi(\psi \in \mathfrak{S})$ and therefore $\lim _{n \rightarrow \infty} \tilde{A}_{n} \tilde{\psi}$ $=\tilde{\psi}(\tilde{\psi} \in \tilde{\mathfrak{W}})$. Hence $\tilde{V}$ is dense in $\widetilde{\mathfrak{W}}$. If $X \in \mathfrak{g}_{0}$ and $f \in C_{c}^{\infty}(G)$ we denote by $X f$ 
the function $x \rightarrow\{d f(x \exp t X) / d t\}_{t \rightarrow 0}$. This defines a representation of $\mathfrak{g}_{0}$ on $C_{c}^{\infty}(G)$ which may be extended uniquely to a representation of $\mathfrak{B}$. Let $\sigma(x)$ denote the adjoint of $\pi\left(x^{-1}\right)$. Then if $\psi \in \mathfrak{S}$ and $\tilde{\psi} \in \tilde{\mathfrak{E}}$ we find that

$$
\begin{aligned}
\lim _{t \rightarrow 0} \frac{1}{t}\left(\sigma(\exp t X) \tilde{A} f \tilde{\psi}-\tilde{A}_{f} \tilde{\psi}, \psi\right) & =\lim _{t \rightarrow 0} \frac{1}{t}\left(\tilde{\psi}, \pi(\exp -t X) A_{f} \psi-A_{f} \psi\right) \\
& =\left(\tilde{\psi}, A_{-x,} \psi\right)=\left(\tilde{A}_{-X f} \tilde{\psi}, \psi\right) .
\end{aligned}
$$

This shows that

$$
\lim _{t \rightarrow 0} \frac{1}{t}\left(\sigma(\exp t X) \tilde{A} f \tilde{\psi}-\tilde{A}_{f} \tilde{\psi}\right)=\tilde{A}_{-X j} \tilde{\psi}
$$

and therefore we get a representation $\sigma \tilde{V}$ of $\mathfrak{B}$ on $\tilde{V}$ such that

$$
\sigma \tilde{V}(X) \tilde{\psi}=\lim _{t \rightarrow 0} \frac{1}{t}(\sigma(\exp t X) \tilde{\psi}-\tilde{\psi}) \quad\left(\tilde{\psi} \in \tilde{V}, X \in g_{0}\right) .
$$

Hence

$$
\begin{aligned}
(\sigma \tilde{V}(X) \tilde{\psi}, \psi) & =\lim _{t \rightarrow 0} \frac{1}{t}(\sigma(\exp t X) \tilde{\psi}-\tilde{\psi}, \psi) \\
& =\lim _{t \rightarrow 0} \frac{1}{t}(\tilde{\psi}, \pi(\exp -t X) \psi-\psi)=\left(\tilde{\psi}, \pi_{V}(-X) \psi\right)
\end{aligned}
$$

if $\psi \in U, \tilde{\psi} \in \tilde{V}$ and $X \in \mathfrak{g}_{0}$. Let $\phi$ denote the antiautomorphism of $\mathfrak{B}$ (over $C$ ) such that $\phi(X)=-X(X \in \mathfrak{g})$. Then $\left(\tilde{\psi}, \pi_{U}(b) \psi\right)=\left(\sigma_{\tilde{V}}(\phi(b)) \tilde{\psi}, \psi\right) \quad(\psi \in U$, $\tilde{\psi} \in \tilde{V}, b \in \mathfrak{B})$. Now let $z$ be any element in $\mathbb{Z}$ and $B$ the restriction of the operator $\pi_{U}(z)$ on $U_{0}$. Then $B$ is $\chi(z)$ times the unit operator and so is bounded. On the other hand, $\tilde{V}$ is dense in $\widetilde{\mathfrak{W}}$ and $\left(\tilde{\psi}, \pi_{U}(z) \psi\right)=(\sigma \widetilde{V}(\phi(z) \tilde{\psi}, \psi)$ $(\tilde{\psi} \in \tilde{V}, \psi \in U)$. Since $U_{0}$ is dense in $\mathfrak{S}$ we conclude from Lemma 33 that $\pi_{U}(z)$ is also bounded. Let $A$ denote the unique bounded extension of the operator $\pi_{U}(z)$ on $\mathfrak{S}$. Then $A-\chi(z) I$ is bounded and since it is zero on $U_{0}$ it must be zero. Therefore $\pi_{U}(z) \psi=A \psi=\chi(z) \psi$ for $\psi \in U$. This proves the lemma.

Let $\pi$ be a representation of $G$ on $\mathfrak{S}$ and $U$ any linear subspace of $\mathfrak{S}$ which is stable under $\pi(K)$. Then for any $\mathfrak{D} \in \Omega$, we denote by $U_{\triangleright}$ the set of all elements in $U$ which transform under $\pi(K)$ according to $\mathfrak{D}$.

LeMma 33. Let $\pi$ be a quasi-simple representation of $G$ on $\mathfrak{W}$ and $\pi_{W}$ the corresponding representation of $\mathfrak{B}$ on the space $W$ of all well-behaved elements. Suppose $\psi_{0} \in \sum_{\mathbb{D} \in \Omega} W_{\triangleright}$ and $U=\mathrm{Cl}\left(\pi_{W}(\mathfrak{B}) \psi_{0}\right)$ (where $\mathrm{Cl}$ denotes closure in $\mathfrak{S )}$. Then $U$ is invariant under $\pi(G), \pi_{W}(\mathfrak{B}) \psi_{0}=\sum_{\mathbb{Q} \in \Omega} U_{\mathbb{D}}$, and $\operatorname{dim} U_{\triangleright}<\infty$ $(\mathfrak{D} \in \Omega)$.

We use the notation of Part I and denote by $\mathfrak{l}$ the subspace of $\mathfrak{g}$ spanned by $\mathfrak{l}_{0}$ over $C$. Let $\mathfrak{X}$ be the subalgebra of $\mathfrak{B}$ generated by $(1, \mathfrak{l})$. Since $K$ is 
simply-connected there is a 1-1 correspondence between finite-dimensional irreducible representations of $\mathfrak{l}_{0}$ (and therefore of $\mathfrak{t}$ or $\mathfrak{X}$ ) and those of $K$. Hence any $\mathfrak{D} \in \Omega$ may be looked upon as an equivalence class of finite-dimensional irreducible representations of $\mathfrak{t}$. We shall regard it in this way whenever it is convenient to do so.

We already know from the corollary to Theorem 2 that $U$ is stable under $\pi(G)$. Put $U_{0}=\pi_{W}(\mathfrak{B}) \psi_{0}$. It follows from Lemma 9 that $U_{0} \subset \sum_{\triangleright \in \Omega} W_{\triangleright}$ and therefore $U_{0}=\sum_{\mathscr{D} \in \Omega} V_{0} \cap W_{\triangleright}$ (Lemma 6). Let $\mathfrak{V}$ be the set of all elements $x \in \mathfrak{X}$ such that $\pi_{W}(x) \psi_{0}=0$. Then $\mathfrak{Y}$ is a left ideal in $\mathfrak{X}$ which satisfies the conditions of Theorem 1. Let $a \rightarrow a^{*}(a \in \mathfrak{B})$ denote the natural mapping and $\pi^{*}$ the natural representation of $\mathscr{B}$ on $\mathfrak{B}^{*}=\mathfrak{B} / \mathfrak{B} \mathfrak{Y}$. Put $\alpha\left(b^{*}\right)=\pi_{W}(b) \psi_{0}(b \in \mathfrak{B})$. Then $b^{*} \rightarrow \alpha\left(b^{*}\right)$ is a well-defined mapping of $\mathfrak{B}^{*}$ into $U_{0}$ and $\alpha\left(\pi^{*}(a) b^{*}\right)$ $=\pi_{W}(a) \alpha\left(b^{*}\right)(a, b \in \mathfrak{B})$. Now $\mathfrak{B}^{*}=\sum \mathfrak{D} \in \Omega \mathfrak{B}_{\mathfrak{D}}^{*}$ in the notation of Theorem 1 and therefore $\alpha\left(\mathfrak{B}_{\mathfrak{D}}^{*}\right)=U_{0} \cap W_{\mathfrak{D}}(\mathfrak{D} \in \Omega)$. Moreover for every $\mathfrak{D} \in \Omega$ we can choose a finite set of elements $b_{1}{ }^{*}, \cdots, b_{k}^{*} \in \mathfrak{B}_{\mathfrak{D}}^{*}$ such that $\mathfrak{B}_{\mathfrak{D}}^{*}$ $=\sum_{i=1}^{k} \pi^{*}(3) b_{i}^{*}$. Hence $U_{0} \cap W_{\mathbb{D}}=\sum_{i=1}^{k} \pi_{W}(3) \alpha\left(b_{i}^{*}\right)$. But since $\pi$ is quasisimple, it follows from the corollary to Lemma 32 that $\pi_{W}(z)=\chi(z) \pi_{W}(1)$ $(z \in 3)$ where $\chi$ is the infinitesimal character of $\pi$. Therefore $\alpha\left(b_{i}^{*}\right), 1 \leqq i \leqq k$, span $U_{0} \cap W_{\triangleright}$ and so $\operatorname{dim}\left(U_{0} \cap W_{\mathfrak{D}}\right)<\infty$. Now $U_{0} \cap W_{\mathfrak{D}}=U_{0} \cap \mathfrak{Q}_{\mathfrak{D}}=U_{0} \cap U_{\mathfrak{D}}$. Since $U_{0}=\sum_{\Phi \in \Omega} U_{0} \cap U_{\triangleright}$ is dense in $U$, it follows from Lemma 30 that $U_{D}=U_{0} \cap U_{D}$. But the dimension of $U_{0} \cap U_{D}$ is finite and therefore $U_{D}$ $=U_{0} \cap U_{D}$. Hence $U_{0}=\sum_{\mathbb{E} \in \Omega} U_{\triangleright}$ and $\operatorname{dim} U_{\triangleright}<\infty$.

Lemma 34. Let $\pi$ be a permissible representation of $G$ on $\mathfrak{S}$ such that $\operatorname{dim} \mathfrak{E}_{\mathbb{D}}<\infty$ for every $\mathfrak{D} \in \Omega$. Then every element $\psi_{0}$ in $\sum_{\mathbb{D} \in \Omega} \mathfrak{S}_{\mathbb{D}}$ is wellbehaved and $\pi_{W}(\mathfrak{B}) \psi_{0}=\sum \sum_{\mathbb{Q} \in \Omega} U_{\triangleright}$ where $U=\mathrm{Cl}\left(\pi_{W}(\mathfrak{B}) \psi_{0}\right)$.

We know from Theorem 4 and Lemma 31 that $\mathfrak{S}_{\mathfrak{D}}=\bar{W}_{\mathfrak{D}}$. But since $\operatorname{dim} \mathfrak{S}_{\mathfrak{D}}<\infty, \mathfrak{S}_{\mathfrak{D}}=W_{\mathfrak{D}}$ and therefore $\psi_{0}$ is well-behaved. Put $V=\pi_{W}(\mathfrak{B}) \psi_{0}$ and $V_{\mathbb{D}}=V \cap U_{\mathfrak{D}}$. Then $\psi_{0} \in \sum_{\mathbb{D} \in \Omega} V_{\mathbb{D}}$ and therefore, from Lemma 6 , $V=\sum_{\triangleright \in \Omega} V_{\triangleright}$. Since $V$ is dense in $U$ we conclude from Lemma 31 that $V_{\triangleright}$ is dense in $U_{\mathfrak{D}}$. But $\operatorname{dim} V_{\mathfrak{D}} \leqq \operatorname{dim} \mathfrak{S}_{\mathfrak{D}}<\infty$. Hence $U_{\mathfrak{D}}=V_{\triangleright}$ and therefore $V=\sum \mathbb{D} \in \Omega \quad U_{\mathfrak{D}}$.

The following theorem is of decisive significance for our purpose.

TheOREM 5. Let $\pi$ be a permissible representation of $G$ on $\mathfrak{S}$ such that $\operatorname{dim} \mathfrak{S}_{\mathfrak{D}}<\infty$ for all $\mathfrak{D} \in \Omega$. For any $\psi_{0} \in \sum_{\mathfrak{D} \in \Omega} \mathfrak{S}_{\mathfrak{D}}$ put $U^{\prime}=\pi_{W}(\mathfrak{B}) \psi_{0}$ and $U=\mathrm{Cl}\left(U^{\prime}\right)$. Let $M$ be the set of all closed linear subspaces of $U$ which are invariant under $\pi(G)$. Similarly let $M^{\prime}$ be the set of all linear subspaces of $U^{\prime}$ which are invariant under $\pi_{W}(\mathfrak{B})$. Then there exists a 1-1 mapping $V \rightarrow V^{\prime}$ of $M$ onto $M^{\prime}$ such that $V^{\prime}=V \cap U^{\prime}, V=\mathrm{Cl}\left(V^{\prime}\right)$, and $V^{\prime}=\sum_{\triangleright \in \Omega} V_{\triangleright}$.

Notice that since $\operatorname{dim} \mathfrak{S}_{\mathbb{D}}<\infty$ it follows from Lemma 34 that $\psi_{0}$ is wellbehaved, $U$ is invariant under $\pi(G)$, and $U^{\prime}=\sum_{\mathbb{D}^{\Omega}} U_{\mathfrak{D}}$. For any $V \in M$ put $V^{\prime}=V \cap U^{\prime}$. Then if $\psi \in V^{\prime}$ and $X \in g_{0}$, 


$$
\pi_{W}(X) \psi=\lim _{t \rightarrow 0} \frac{1}{t}\{\pi(\exp t X) \psi-\psi\} \in V \cap U^{\prime}=V^{\prime}
$$

since $V$ is closed. Hence $V$ is stable under $\pi_{W}(\mathfrak{B})$ and therefore, from Lemma 6, $V^{\prime}=\sum_{\mathfrak{D} \in \Omega} V^{\prime} \cap U_{\mathfrak{D}}=\sum_{\mathfrak{D} \in \Omega} V_{\mathfrak{D}}$. Moreover $\sum_{\mathfrak{D} \in \Omega} V_{\mathfrak{D}}$ is dense in $V$ (Theorem 4). Hence $V=\mathrm{Cl}\left(V^{\prime}\right)$.

Conversely let $V^{\prime} \in M^{\prime}$. Then, again from Lemma $6, V^{\prime}=\sum_{\mathfrak{D} \in \Omega} V^{\prime} \cap U_{\mathfrak{D}}$. Since every element in $V^{\prime}$ is well-behaved $V=\mathrm{Cl}\left(V^{\prime}\right)$ is invariant under $\pi(G)$. But then, $V_{\mathfrak{D}}=\mathrm{Cl}\left(V^{\prime} \cap U_{\mathfrak{D}}\right) \quad\left(\right.$ Lemma 30). Since $\operatorname{dim}\left(V^{\prime} \cap U_{\mathfrak{D}}\right)$ $\leqq \operatorname{dim} \mathfrak{S}_{\mathfrak{D}}<\infty, V_{\mathfrak{D}}=V^{\prime} \cap U_{\mathfrak{D}}$ and so $V^{\prime}=\sum_{\mathfrak{D} \in \Omega} V_{\mathfrak{D}}$. Moreover since $V \in M$ we know from the earlier part of the proof that $V \cap U^{\prime}=\sum_{\mathfrak{D} \in \Omega} V_{\mathfrak{D}}$. Therefore $V \cap U^{\prime}=V^{\prime}$ and the theorem is proved.

Let $\mathfrak{S}_{1}, \mathfrak{W}_{2}$ be two closed subspaces of $\mathfrak{S}$ both invariant under $\pi(G)$. We say that $\mathfrak{W}_{2}$ is maximal in $\mathfrak{W}_{1}$ (with respect to $\pi$ ) if (11) $\mathfrak{W}_{1}>\mathfrak{W}_{2}$ and there exists no closed subspace $\mathfrak{W}_{3}$ invariant under $\pi(G)$ such that $\mathfrak{S}_{1}>\mathfrak{S}_{3}>\mathfrak{W}_{2}$. Then we get the following corollary from Theorem 5 .

CoRollary 1. If $U \neq\{0\}$ there exists an element $V \in M$ such that $V$ is maximal in $U$.

Let $\mathfrak{M}_{0}$ be the left ideal in $\mathfrak{B}$ consisting of all elements $b \in \mathfrak{B}$ such that $\pi_{W}(b) \psi_{0}=0$. Since $U \neq\{0\}, \psi_{0} \neq 0$ and therefore $1 \in \mathfrak{M}_{0}$. Therefore by Zorn's lemma there exists a maximal left ideal $\mathfrak{M}$ in $\mathfrak{B}$ containing $\mathfrak{M}_{\mathbf{0}}$. Put $V^{\prime}$ $=\pi_{W}(\mathfrak{M}) \psi_{0}$. Then it is clear that $V^{\prime}$ is not properly contained in any element of $M^{\prime}$ other than $U^{\prime}$. Put $V=\mathrm{Cl}\left(V^{\prime}\right)$. We claim $V$ is maximal in $U$. For let $U \supset S \supset V(S \in M)$. Then $U^{\prime} \supset S^{\prime} \supset V^{\prime}$ and therefore $S^{\prime}=U^{\prime}$ or $V^{\prime}$. Since the mapping from $M$ to $M^{\prime}$ is 1-1 this proves that $S=U$ or $V$ and $U \neq V$ and this establishes our assertion.

COROllary 2. Let $\pi$ be a quasi-simple representation of $G$ on $\mathfrak{S} \neq\{0\}$. Then it is possible to find two closed invariant subspaces $U$ and $V$ in $\mathfrak{S}$ such that $V$ is maximal in $U$.

As before let $W$ be the space of well-behaved elements in $\mathfrak{S}$. Then $\sum_{\mathfrak{D} \in \Omega} W_{\mathfrak{D}}$ is dense in $\mathfrak{S}$ (Theorem 4). Choose $\psi_{0} \in \sum_{\mathfrak{D} \in \Omega} W_{\mathfrak{D}}, \psi_{0} \neq 0$, and put $U=\mathrm{Cl}\left(\pi_{W}(\mathfrak{B}) \psi_{0}\right)$. From Lemma 33, $\operatorname{dim} U_{\mathfrak{D}}<\infty(\mathfrak{D} \in \Omega)$ and so our result follows immediately from Corollary 1 above.

Let $\pi_{1}, \pi_{2}$ be two representations of $G$ on the Banach spaces $\mathfrak{S}_{1}$ and $\mathfrak{W}_{2}$ respectively. We say that they are equivalent if there exists a linear mapping $S$ which maps $\mathfrak{S}_{1}$ topologically onto $\mathfrak{S}_{2}$ such that $\pi_{2}(x) S=S \pi_{1}(x)$ for all $x \in G$. However in case $\pi_{1}$ and $\pi_{2}$ are both permissible we introduce the concept of a new kind of equivalence as follows. Let $W_{i}$ be the space of all well-behaved elements in $\mathfrak{S}_{i}$, and let $\pi_{W i}$ be the representation of $\mathfrak{B}$ on $W_{i}(i=1,2)$. Put

(11) $A$ and $B$ being two sets we write $A>B$ or $A<B$ if $B \supset A$ and $A \neq B$. 
$W_{i, \mathscr{D}}=W_{\boldsymbol{i}} \cap\left(\mathfrak{Y}_{i}\right)_{\mathfrak{D}}$ and $\mathfrak{S}_{\mathfrak{i}}^{0}=\sum_{\mathscr{D} \in \Omega} W_{\boldsymbol{i}, \mathfrak{D}}$. Then we know from Theorem 4 and Lemma 9 that $\mathfrak{S}_{i}^{0}$ is dense in $\mathfrak{S}_{i}$, and it is stable under $\pi_{W_{i}}(\mathfrak{B})$. Let $\pi_{i}^{0}$ denote the representation of $\mathfrak{B}$ induced on $\mathfrak{S}_{\mathfrak{i}}^{0}$. We shall say that the representations $\pi_{1}, \pi_{2}$ are infinitesimally equivalent if there exists a 1-1 linear mapping $\alpha$ of $\mathfrak{S}_{1}^{0}$ onto $\mathfrak{S}_{2}^{0}$ such that $\pi_{2}^{0}(b) \alpha(\psi)=\alpha\left(\pi_{1}^{0}(b) \psi\right)$ for all $b \in \mathfrak{B}$ and $\psi \in \mathfrak{S}_{1}^{0}$. It is clear that if $\pi_{1}, \pi_{2}$ are equivalent they are also infinitesimally equivalent, but the converse is not true in general. However we shall see later $(\$ 11)$ that for irreducible unitary representations on Hilbert spaces these two concepts of equivalence actually coincide.

11. Unitary representations on a Hilbert space. In this section $\mathfrak{S}$ shall stand for a Hilbert space. A representation $\pi$ of $G$ on $\mathfrak{S}$ is called unitary if the operator $\pi(x)$ is unitary for every $x \in G$. For any two elements $\phi, \psi$ in $\mathfrak{E}$ we denote by $(\phi, \psi)$ their scalar product.

THEOREM 6. Let $\pi$ be an irreducible representation of $G$ on a Hilbert space $\mathfrak{S}$. Then every element in $\sum_{\mathbb{D} \in \Omega} \mathfrak{S}_{\mathfrak{D}}$ is well-behaved and $\operatorname{dim} \mathfrak{D}_{\mathfrak{D}}<\infty(\mathfrak{D} \in \Omega)$. Moreover $\sum_{\mathfrak{D} \in \Omega} \mathfrak{S}_{\mathfrak{D}}$ is dense in $\mathfrak{S}$.

It is known that an irreducible unitary representation of $G$ on $\mathfrak{S}$ is quasisimple (see Mautner [12] and Segal [15]). Let $W$ be the space of all wellbehaved elements in $\mathfrak{S}$. Then $\sum_{\mathfrak{D} \in \Omega} W_{\triangleright}$ is dense in $\mathfrak{S}$ (Theorem 4). Choose $\psi_{0} \in \sum_{\mathscr{D} \in \Omega} W_{\mathfrak{D}}, \psi_{0} \neq 0$. Since $\pi$ is irreducible it follows from Lemma 33 that $\mathfrak{S}=\mathrm{Cl}\left(\pi_{W}(\mathfrak{B}) \psi_{0}\right), \pi_{W}(\mathfrak{B}) \psi_{0}=\sum_{\mathfrak{D} \in \mathfrak{\Omega}} \mathfrak{S}_{\mathfrak{D}}$, and $\operatorname{dim} \mathfrak{S}_{\mathfrak{D}}<\infty$. This proves the theorem.

THEOREM 7. Let $\pi$ be a quasi-simple unitary representation of $G$ on $\mathfrak{S} \neq\{0\}$. Then there exists a minimal $\left.{ }^{12}\right)$ closed invariant subspace in $\mathfrak{S}$.

From Corollary 2 of Theorem 5 we can choose two closed invariant subspaces $U$ and $S$ such that $S$ is maximal in $U$. Let $V$ be the orthogonal complement of $S$ in $U$. Since $\pi$ is unitary it is clear that $V$ is invariant and minimal.

The above theorem has the following significance in relation to the theory of Murray and von Neumann [14]. Let $\pi$ be a unitary representation of $G$ on $\mathfrak{E}$ and $\mathfrak{A}$ the smallest weakly closed algebra of bounded operators on $\mathfrak{S}$ which contains $\pi(G)$. Suppose $\mathfrak{A}$ is a factor, i.e. the center of $\mathfrak{A}$ consists of scalar multiples of the unit operator. Then it can be shown that $\pi$ must be quasi-simple and we conclude from Theorem 7 that there exists a closed subspace $V \neq\{0\}$ which is invariant and irreducible under $\pi(G)$ and therefore under $\mathfrak{A}$. But then it follows from the results of Murray and von Neumann (Lemmas 5.3.1, 5.3.8, 8.6.1) that $\mathfrak{A}$ is of type $\mathrm{I}_{n}$ or $\mathrm{I}_{\infty}$. Thus we have proved that any factor arising from a unitary representation of semisimple Lie group in a Hilbert space is necessarily of type I.

The following theorem shows that for irreducible unitary representations infinitesimal equivalence is the same as ordinary equivalence.

$\left.{ }^{(12}\right)$ A closed invariant subspace $V$ is called minimal if $\{0\}$ is maximal in $V$. 
THEOREM 8. Let $\pi_{1}, \pi_{2}$ be two irreducible unitary representations of $G$ on the Hilbert spaces $\mathfrak{S}_{1}$ and $\mathfrak{S}_{2}$ respectively. Then they are infinitesimally equivalent if and only if they are equivalent.

It follows from Theorem 6 that $\operatorname{dim} \mathfrak{S}_{i, \mathfrak{D}}<\infty(\mathfrak{D} \in \Omega)$, every element in

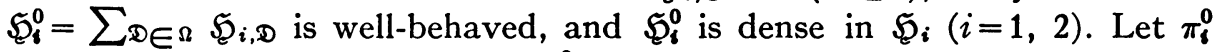
denote the representation of $\mathfrak{B}$ on $\mathfrak{W}_{\mathfrak{l}}^{0}$. Then if $\pi_{1}, \pi_{2}$ are infinitesimally equivalent there exists a 1-1 linear mapping $\alpha$ of $\mathfrak{S}_{1}^{0}$ onto $\mathfrak{E}_{2}^{0}$ such that $\alpha\left(\pi_{1}^{0}(b) \psi\right)$ $=\pi_{2}^{0}(b) \alpha \psi$ for all $\psi \in \mathfrak{S}_{1}^{0}$ and $b \in \mathfrak{B}$. It is clear that $\alpha\left(\mathfrak{W}_{1, D}\right)=\mathfrak{W}_{2, \mathbb{D}}(\mathfrak{D} \in \Omega)$ and the representations of $K$ induced on $\mathfrak{E}_{1, D}$ and $\mathfrak{E}_{2, D}$ are unitary and equivalent to each other. Therefore there exists a 1-1 linear mapping $\beta_{D}$ of $\mathfrak{W}_{1, D}$ onto $\mathfrak{W}_{2, D}$ such that if $\phi_{1}, \psi_{1} \in \mathfrak{W}_{1, D}$ and $\phi_{2}=\beta_{D} \phi_{1}, \psi_{2}=\beta_{D} \psi_{1}$ then $\left(\phi_{1}, \pi_{1}(u) \psi_{1}\right)=\left(\phi_{2}, \pi_{2}(u) \psi_{2}\right)$ for all $u \in K$. Let $\beta$ be the 1-1 linear mapping of $\mathfrak{W}_{1}^{0}$ onto $\mathfrak{W}_{2}^{0}$ such that $\beta$ coincides with $\beta_{\mathfrak{D}}$ on $\mathfrak{W}_{1, \mathfrak{D}}$. Now it is easily seen that for two distinct elements $\mathfrak{D}, \mathfrak{D}^{\prime} \in \Omega$ the spaces $\mathfrak{S}_{i, \mathscr{D}}$ and $\mathfrak{S}_{i, \mathscr{D}^{\prime}}$ are mutually orthogonal and therefore $\left(\phi, \pi_{1}(u) \psi\right)=\left(\beta \phi, \pi_{2}(u) \beta \psi\right)$ for all $\phi, \psi \in \mathfrak{W}_{1}^{0}$ and $u \in K$. In particular $|\psi|=|\beta \psi|\left(\psi \in \mathfrak{S}_{1}^{0}\right)$ and since $\mathfrak{W}_{i}^{0}$ is dense in $\mathfrak{S}_{i}$ it follows that $\beta$ can be extended uniquely to an isometric mapping of $\mathfrak{W}_{1}$ onto $\mathfrak{W}_{2}$. Let $S$ denote the linear transformation of $\mathfrak{W}_{1}^{0}$ onto itself given by $S \psi=\beta^{-1} \alpha \psi$ $\left(\psi \in \mathfrak{S}_{1}^{0}\right)$. Since $\alpha$ and $\beta$ are isomorphisms of $\mathfrak{S}_{1}^{0}$ onto $\mathfrak{S}_{2}^{0}$ it follows that the inverse transformation $S^{-1}=\alpha^{-1} \beta$ exists. Moreover it is obvious that $S$ and $S^{-1}$ leave $\mathfrak{S}_{1, \mathbb{D}}$ invariant $(\mathfrak{D} \in \Omega)$. Since $\operatorname{dim} \mathfrak{S}_{1, \mathcal{D}}<\infty$ it follows immediately that there exists a linear transformation $S^{*}$ of $\mathfrak{W}_{1}^{0}$ into itself such that

$$
\left(S^{*} \phi, \psi\right)=(\phi, S \psi) \quad\left(\phi, \psi \in \mathfrak{W}_{1}^{0}\right) .
$$

Furthermore the inverse transformation $S^{*-1}$ exists and $S^{*}, S^{*-1}$ leave $\mathfrak{W}_{1, \mathfrak{D}}$ invariant for all $\mathfrak{D} \in \Omega$. Put $A=S^{*} S$. Then we claim that

$$
A \pi_{1}^{0}(b) A^{-1}=\pi_{1}^{0}(b)
$$

First notice that if $X \in \mathfrak{g}_{0}$ and $\phi_{i}, \psi_{i} \in \mathfrak{W}_{i}^{0}(i=1,2)$ then

$$
\begin{aligned}
\left(\phi_{i}, \pi_{i}^{0}(X) \psi_{i}\right) & =\lim _{t \rightarrow 0} \frac{1}{t}\left(\phi_{i}, \pi_{i}(\exp t X) \psi_{i}-\psi_{i}\right) \\
& =\lim _{t \rightarrow 0} \frac{1}{t}\left(\pi_{i}(\exp -t X) \phi_{i}-\phi_{i}, \psi_{i}\right)=-\left(\pi_{i}^{0}(X) \phi_{i}, \psi_{i}\right)
\end{aligned}
$$

since $\pi_{i}$ is a unitary representation. Hence if $\phi, \psi \in \mathfrak{F}_{1}^{0}$,

$$
\begin{aligned}
\left(\phi, A \pi_{1}^{0}(X) A^{-1} \psi\right) & =\left(\phi, S^{*} S \pi_{1}^{0}(X) S^{-1} S^{*-1} \psi\right) \\
& =\left(S \phi, S \pi_{1}^{0}(X) S^{-1} S^{*-1} \psi\right) \\
& =\left(\beta S \phi, \pi_{2}^{0}(X) \beta S^{*-1} \psi\right)
\end{aligned}
$$

since $\beta S \pi_{1}^{0}(X) S^{-1}=\alpha \pi_{1}^{0}(X) \alpha^{-1} \beta=\pi_{2}^{0}(X) \beta$. Therefore 


$$
\begin{aligned}
\left(\phi, A \pi_{1}^{0}(X) A^{-1} \psi\right) & =\left(\alpha \phi, \pi_{2}^{0}(X) \beta S^{*-1} \psi\right)=-\left(\pi_{2}^{0}(X) \alpha \phi, \beta S^{*-1} \psi\right) \\
& =-\left(\alpha \pi_{1}^{0}(X) \phi, \beta S^{*-1} \psi\right) \\
& =-\left(\beta^{-1} \alpha \pi_{1}^{0}(X) \phi, S^{*-1} \psi\right) \\
& =-\left(S \pi_{1}^{0}(X) \phi, S^{*-1} \psi\right) \\
& =-\left(\pi_{1}^{0}(X) \phi, \psi\right)=\left(\phi, \pi_{1}^{0}(X) \psi\right) .
\end{aligned}
$$

Since this is true for all $\phi \in \mathfrak{S}_{1}^{0}$ and since $\mathfrak{W}_{1}^{0}$ is dense in $\mathfrak{W}_{1}$ we conclude that

$$
A \pi_{1}^{0}(X) A^{-1} \psi=\pi_{1}^{0}(X) \psi \quad\left(\psi \in \mathfrak{S}_{1}^{0}, X \in \mathfrak{g}_{0}\right)
$$

and from this our assertion follows immediately.

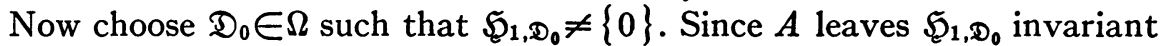
and since $\operatorname{dim} \mathfrak{E}_{1, D_{0}}<\infty$ we can find an element $\psi_{0} \in \mathfrak{W}_{1, D_{0}}\left(\psi_{0} \neq 0\right)$ and a complex number $c$ such that $A \psi_{0}=c \psi_{0}$. But $c\left|\psi_{0}\right|^{2}=\left(\psi_{0}, S^{*} S \psi_{0}\right)=\left|S \psi_{0}\right|^{2}$ and $S \psi_{0} \neq 0$. Therefore $c$ is real and positive. Moreover $A \pi_{1}^{0}(b) \psi_{0}=\pi_{2}^{0}(b) A \psi_{0}$ $=c \pi_{1}^{0}(b) \psi_{0}(b \in \mathfrak{B})$. Let $V=\pi_{1}^{0}(\mathscr{B}) \psi_{0}$. Since $\pi_{1}$ is irreducible it follows from Lemma 33 that $\bar{V}=\mathfrak{S}_{1}$ and $V=\sum_{\mathscr{D} \in \Omega} \mathfrak{W}_{1, D}=\mathfrak{W}_{1}^{0}$. Therefore $A \psi=c \psi$ for all $\psi \in \mathfrak{S}_{1}^{0}$. Now $|\alpha \psi|^{2}=|S \psi|^{2}=(\psi, A \psi)=c|\psi|^{2}\left(\psi \in \mathfrak{W}_{1}^{0}\right)$ and so $\alpha$ is continuous. Hence it may be extended uniquely to a linear mapping of $\mathfrak{S}_{1}$ into $\mathfrak{S}_{2}$ such that $|\alpha \psi|=c^{1 / 2}|\psi|\left(\psi \in \mathfrak{W}_{1}\right)$. Since $c>0, \alpha$ maps $\mathfrak{S}_{1}$ topologically into $\mathfrak{W}_{2}$ and therefore $\alpha \mathfrak{W}_{1}$, being complete, is closed in $\mathfrak{S}_{2}$. But $\alpha \mathfrak{W}_{1} \supset \mathfrak{E}_{2}^{0}$ and $\mathfrak{S}_{2}^{0}$ is dense in $\mathfrak{S}_{2}$. Hence $\alpha \mathfrak{S}_{1}=\mathfrak{S}_{2}$.

Now let $\psi \in \mathfrak{S}_{1}^{0}$. Then by Theorem 2 we can choose a neighbourhood $V$ of zero in $g_{0}$ such that if $X \in V, \pi_{1}(\exp X) \psi=\sum_{n \geq 0}(1 / n !) \pi_{1}^{0}\left(X^{n}\right) \psi$ and $\pi_{2}(\exp X) \alpha \psi=\sum_{n \geq 0}(1 / n !) \pi_{2}^{0}\left(X^{n}\right) \alpha \psi$. But since $\alpha$ is a continuous linear mapping we find that

$$
\alpha \pi_{1}(\exp X) \psi=\sum_{n \geqq 0} \frac{1}{n !} \alpha \pi_{1}^{0}\left(X^{n}\right) \psi=\sum_{n \geqq 0} \frac{1}{n !} \pi_{2}^{0}\left(X^{n}\right) \alpha \psi=\pi_{2}(\exp X) \alpha \psi
$$

$(X \in V)$.

Put $\psi(X)=\alpha \pi_{1}(\exp X) \psi-\pi_{2}(\exp X) \alpha \psi\left(X \in \mathfrak{g}_{0}\right)$. It is obvious that the mapping $X \rightarrow \psi(X)$ is analytic. Hence if $\phi$ is an element in $\mathfrak{W}_{2},(\phi, \psi(X))$ is an analytic function on $\mathfrak{g}_{0}$ which is zero on $V$. This however implies that $(\phi, \psi(X))=0$ for all $X \in \mathfrak{g}_{0}$. This being true for every $\phi \in \mathfrak{W}_{2}$ it follows that $\psi(X)=0\left(X \in \mathfrak{g}_{0}\right)$. Hence $\alpha \pi_{1}(\exp X) \psi=\pi_{2}(\exp X) \alpha \psi\left(X \in \mathfrak{g}_{0}, \psi \in \mathfrak{W}_{1}^{0}\right)$. But $\mathfrak{S}_{1}^{0}$ is dense in $\mathfrak{W}_{1}$, and therefore this relation is actually true for all $\psi \in \mathfrak{W}_{1}$. Moreover since $G$ is generated by the elements $\exp X\left(X \in \mathfrak{g}_{0}\right)$, it follows that $\alpha \pi_{1}(x) \psi=\pi_{2}(x) \alpha \psi\left(x \in G, \psi \in \mathfrak{S}_{1}\right)$ and therefore $\pi_{1}, \pi_{2}$ are equivalent. Conversely, if $\pi_{1}, \pi_{2}$ are equivalent, it is obvious that they are infinitesimally equivalent. Hence the theorem.

Let $\pi$ be a permissible representation of $G$ on a Banach space $\mathfrak{S}$ and let $W$ be the space of all well-behaved elements in $\mathfrak{g}$. Put $\mathfrak{\bigotimes}_{0}=\sum \sum_{\mathbb{Q} \in \mathfrak{\Omega}} W_{\mathfrak{D}}$. We 
know that $\mathfrak{g}_{0}$ is stable under the representation of $\mathfrak{B}$ on $W$ (Lemma 9 ). Let $\pi_{0}$ denote the representation of $\mathfrak{B}$ on $\mathfrak{E}_{0}$. We shall say that $\pi$ is infinitesimally unitary if it is possible to define a scalar product $(\psi, \phi)\left(\psi, \phi \in \mathfrak{W}_{0}\right)$ such that under this product $\mathfrak{S}_{0}$ becomes a (possibly incomplete) Hilbert space and $\left(\psi, \pi_{0}(X) \phi\right)=-\left(\pi_{0}(X) \psi, \phi\right)$ for all $\psi, \phi \in \mathfrak{g}_{0}$ and $X \in \mathfrak{g}_{0}$.

THEOREм 9. Let $\pi$ be a quasi-simple irreducible representation of $G$ on a Banach space. Then if $\pi$ is infinitesimally unitary, it is infinitesimally equivalent to a unitary representation $\sigma$ of $G$ on a Hilbert space. Moreover, $\sigma$ is unique, apart from equivalence, and it is irreducible.

We keep to the above notation. Then it follows from Lemma 33 that $\mathfrak{S}_{0}=\sum \mathfrak{D}_{\mathbb{Q}} \mathfrak{S}_{\mathfrak{D}}$. Let $(\phi, \psi)$ be the scalar product in $\mathfrak{S}_{0}$ satisfying the abovementioned properties with respect to $\pi_{0}$. We can then complete $\mathfrak{S}_{0}$ with respect to the norm $\|\psi\|=(\psi, \psi)^{1 / 2}\left(\psi \in \mathfrak{S}_{0}\right)$ and thus obtain a (complete) Hilbert space $V$. We shall now define a representation $\sigma$ of $G$ on $V$.

Let $\psi_{0}$ be a fixed element in $\mathfrak{S}_{0}\left(\psi_{0} \neq 0\right)$. Consider the mapping

$$
T:(x, y, z) \rightarrow \pi(x) \pi(y) \pi(z) \psi_{0}=\pi(x y z) \psi_{0} \quad(x, y, z \in G)
$$

of $G \times G \times G$ into $\mathfrak{S}$. Since $\psi_{0}$ is well-behaved since $(x, y, z) \rightarrow x y z$ is an analytic mapping of $G \times G \times G$ into $G, T$ is analytic (Lemma 16). Hence we can find an open convex neighbourhood $U$ of zero in $g_{0}$ such that $U=-U$ and

$$
\pi(\exp r X) \pi(\exp s Y) \pi(\exp t Z) \psi_{0}=\sum_{m, n, p \geqq 0} \psi_{m, n, p}(X, Y, Z) r^{m} s^{n} t^{p}
$$

$\left(\psi_{m, n, p}(X, Y, Z) \in \mathfrak{S}\right)$ for $X, Y, Z \in U$ provided $|r|,|s|,|t| \leqq 1$. Now from the corollary to Lemma 15 we find that

$$
\begin{aligned}
\left\{\frac{\partial^{m}}{\partial r^{m}} \frac{\partial^{n}}{\partial s^{n}} \frac{\partial^{p}}{\partial t^{p}}\left(\pi(\exp r X) \pi(\exp s Y) \pi(\exp t Z) \psi_{0}\right)\right\}_{r=s=t=0} & \\
& =m ! n ! p ! \psi_{m, n, p}(X, Y, Z) .
\end{aligned}
$$

Moreover we know from Theorem 2 that

$$
\pi(\exp t Z) \psi_{0}=\sum_{p \geqq 0} \frac{t^{p}}{p !} \pi_{0}\left(Z^{p}\right) \psi_{0}
$$

if $|t|$ is sufficiently small. Since $\pi(\exp r X) \pi(\exp s Y)$ is a bounded operator,

$$
\pi(\exp r X) \pi(\exp s Y) \pi(\exp t Z) \psi_{0}=\sum_{p} \frac{t^{p}}{p !} \pi(\exp r X) \pi(\exp s Y) \pi_{0}\left(Z^{p}\right) \psi_{0}
$$

Hence

$$
\left\{\frac{\partial^{p}}{\partial t^{p}}\left(\pi(\exp r X) \pi(\exp s Y) \pi(\exp t Z) \psi_{0}\right)\right\}_{t=0}=\pi(\exp r X) \pi(\exp s Y) \pi_{0}\left(Z^{p}\right) \psi_{0}
$$


Again since $\pi_{0}\left(Z^{p}\right) \psi_{0}$ is well-behaved,

$$
\pi(\exp s Y) \pi_{0}\left(Z^{p}\right) \psi_{0}=\sum \frac{s^{n}}{n !} \pi_{0}\left(Y^{n}\right) \pi_{0}\left(Z^{p}\right) \psi_{0}
$$

provided $|s|$ is sufficiently small and so we find in the same way as above that

$$
\left\{\frac{\partial^{n}}{\partial s^{n}}\left(\pi(\exp r X) \pi(\exp s Y) \pi_{0}\left(Z^{p}\right) \psi_{0}\right)\right\}_{8=0}=\pi(\exp r X) \pi_{0}\left(Y^{n}\right) \pi_{0}\left(Z^{p}\right) \psi_{0} .
$$

Repeating the same argument once more we get finally

$$
\begin{aligned}
\left\{\frac{\partial^{m+n+p}}{\partial r^{m} \partial s^{n} \partial t^{p}}\left(\pi(\exp r X) \pi(\exp s Y) \pi(\exp t Z) \psi_{0}\right)\right\}_{r=8=t=0} & \\
& =\pi_{0}\left(X^{m}\right) \pi_{0}\left(Y^{n}\right) \pi_{0}\left(Z^{p}\right) \psi_{0} .
\end{aligned}
$$

Hence

$$
\psi_{m, n, p}(X, Y, Z)=\frac{1}{m ! n ! p !} \pi_{0}\left(X^{m} Y^{n} Z^{p}\right) \psi_{0}
$$

and

$$
\pi(\exp r X) \pi(\exp s Y) \pi(\exp t Z) \psi_{0}=\sum_{m, n, p \geqq 0} \frac{r^{m} s^{n} t^{p}}{m ! n ! p !} \pi_{0}\left(X^{m} Y^{n} Z^{p}\right) \psi_{0}
$$

provided $|r|,|s|,|t| \leqq 1$. Let $Z_{1}, \cdots, Z_{q}$ be a base for $g_{0}$ over $R$. We can choose $\epsilon>0$ such that if $\left|t_{1}\right|, \cdots,\left|t_{q}\right| \leqq \epsilon, Z_{t}=t_{1} Z_{1}+\cdots+t_{q} Z_{q}$ lies in $U$ $\left(t_{i} \in R, 1 \leqq i \leqq q\right)$. Hence

$$
\pi(\exp r X) \pi(\exp s Y) \pi\left(\exp Z_{t}\right) \psi_{0}=\sum_{m, n, p \geqq 0} \frac{r^{m} s^{n}}{m ! n ! p !} \pi_{0}\left(X^{m} Y^{n} Z_{t}^{p}\right) \psi_{0}
$$

But

$$
Z_{t}^{p}=p ! \sum \frac{t_{1}^{p_{1}} t_{2}^{p_{2}} \cdots t_{q}^{p_{q}}}{p_{1} ! p_{2} ! \cdots p_{q} !} Z\left(p_{1}, p_{2}, \cdots, p_{q}\right)
$$

where the sum is over all integers $p_{1}, \cdots, p_{q} \geqq 0$ such that $p_{1}+p_{2}+\cdots$ $+p_{q}=p$ and

$$
Z\left(p_{1}, p_{2}, \cdots, p_{q}\right)=\frac{1}{p !} \sum_{\omega} Z_{i_{\omega(1)}} Z_{i_{\omega(2)}} \cdots Z_{i_{\omega(p)}}
$$

Here $\left\{i_{1}, i_{2}, \cdots, i_{p}\right\}$ is a set of $p$ integers such that exactly $p_{j}$ of them are equal to $j(1 \leqq j \leqq q)$ and the sum is over all permutations $\omega$ of the $p$ integers $(1,2, \cdots, p)$. Therefore 


$$
\begin{aligned}
\left\{\frac{\partial^{p_{1}+\cdots+p_{q}}}{\phi t_{1}^{p_{1}} \cdots \partial t_{q}^{p_{q}}}(\pi(\exp r X) \pi(\exp s Y)\right. & \left.\left.\pi\left(\exp Z_{t}\right) \psi_{0}\right)\right\}_{t_{1}=t_{2}=\cdots-t_{q}=0} \\
& =\sum_{m, n \geqq 0} \frac{r^{m} s^{n}}{m ! n !} \pi_{0}\left(X^{m} Y^{n}\right) \pi_{0}\left(Z\left(p_{1}, \cdots, p_{q}\right)\right) \psi_{0} .
\end{aligned}
$$

Since the elements $Z\left(p_{1}, p_{2}, \cdots, p_{q}\right)$ all taken together span $\mathfrak{B}$ (see [8(a)]) it follows that

$$
\pi(\exp r X) \pi(\exp s Y) \pi_{0}(b) \psi_{0}=\sum_{m, n} \frac{r^{m} s^{n}}{m ! n !} \pi_{0}\left(X^{m} Y^{n}\right) \pi_{0}(b) \psi_{0} \quad(X, Y \in U)
$$

for any $b \in \mathfrak{B}$ and $|r|,|s| \leqq 1$. However, since $\pi$ is quasi-simple and irreducible, it follows from Lemma 33 that $\pi_{0}(\mathfrak{B}) \psi_{0}=\mathfrak{S}_{0}$. Therefore if we let $\dot{r}=s=1$ we get

$$
\pi(\exp X) \pi(\exp Y) \psi=\sum_{m, n \geqq 0} \frac{1}{m ! n !} \pi_{0}\left(X^{m} Y^{n}\right) \psi
$$

for all $\psi \in \mathfrak{S}_{0}$ and $X, Y \in U$. Now put $\exp _{m} Z=1+Z+Z^{2} / 2 !+\cdots+Z^{m} / m$ ! $\in \mathscr{B}$ for any $Z \in g_{0}$. Then the above equation may be written as follows:

$\pi(\exp X) \pi(\exp Y) \psi=\lim _{m \rightarrow \infty, n \rightarrow \infty}(\mathfrak{S}) \pi_{0}\left(\exp _{m} X\right) \pi_{0}\left(\exp _{n} Y\right) \psi\left(X, Y \in U, \psi \in \mathfrak{S}_{0}\right)$.

Here $\lim (\mathfrak{S})$ means limit in $\mathfrak{S}$.

Let $E_{\mathfrak{D}}$ denote the projection of $\mathfrak{S}_{\mathfrak{D}}$ on $\mathfrak{S}_{\mathfrak{D}}$ (see $\$ 8$ ). Since $\mathfrak{S}_{\mathfrak{D}}$ is a finitedimensional subspace of $V$ it may itself be regarded a Hilbert space. Moreover $\mathfrak{S}_{\mathfrak{D}}$ is invariant under $\pi(K)$ and since $\left(\phi_{1}, \pi_{0}(X) \phi_{2}\right)=-\left(\pi_{0}(X) \phi_{1}, \phi_{2}\right) \quad\left(X \in \mathfrak{f}_{0}\right.$, $\left.\phi_{1}, \phi_{2} \in \mathfrak{S}_{\mathfrak{D}}\right)$ it is clear that the representation of $K$ induced on $\mathfrak{S}_{\mathfrak{D}}$ is unitary. From this it follows immediately that if $\mathfrak{D}_{1} \neq \mathfrak{D}_{2}$, then $\mathfrak{S}_{\mathfrak{D}_{1}}$ and $\mathfrak{S}_{\mathfrak{D}_{2}}$ are $\mathrm{mu}$ tually orthogonal subspaces of $\mathfrak{S}_{0}\left(\mathfrak{D}_{1}, \mathfrak{D}_{2} \in \Omega\right)$. Hence $\left(E_{\mathfrak{D}} \phi_{1}, \phi_{2}\right)=\left(\phi_{1}, E_{\mathfrak{D}} \phi_{2}\right)$ $\left(\phi_{1}, \phi_{2} \in \mathfrak{S}_{0}\right)$. For any finite subset $F$ of $\Omega$ put $E_{F}=\sum_{\mathscr{D} \in F} E_{\mathfrak{D}}$. Then $E_{F}$ is a bounded linear operator on $\mathfrak{S}$ and therefore if $\psi \in \mathfrak{S}_{0}$,

$\lim _{m \rightarrow \infty, n \rightarrow \infty},(\mathfrak{S}) E_{F} \pi_{0}\left(\exp _{m} X\right) \pi_{0}\left(\exp _{n} Y\right) \psi=E_{F} \pi(\exp X) \pi(\exp Y) \psi \quad(X, Y \in U)$.

But $E_{F} \mathfrak{W}=\sum_{\mathfrak{D} \in F} \mathfrak{W}_{\mathfrak{D}}$ is a finite-dimensional subspace contained in $\mathfrak{S}_{0}$. Hence the two topologies induced in $E_{F} \mathfrak{S}$ by $\mathfrak{S}$ and $V$ are the same. Therefore

$$
\lim _{m \rightarrow \infty, n \rightarrow \infty}(V) E_{F} \pi_{0}\left(\exp _{m} X\right) \pi_{0}\left(\exp _{n} Y\right) \psi=E_{F} \pi(\exp X) \pi(\exp Y) \psi \quad(X, Y \in U)
$$

where $\lim (V)$ denotes limit in $V$. Now

$$
\begin{aligned}
\left\|\pi_{0}\left(\exp _{m} X\right) \psi\right\|^{2} & =\left(\pi_{0}\left(\exp _{m} X\right) \psi, \pi_{0}\left(\exp _{m} X\right) \psi\right) \\
& =\left(\psi, \pi_{0}\left(\exp _{m}(-X) \exp _{m} X\right) \psi\right) \quad\left(X \in \mathfrak{g}_{0}\right)
\end{aligned}
$$


since $\left(\pi_{0}(Z) \phi_{1}, \phi_{2}\right)=-\left(\phi_{1}, \pi_{0}(Z) \phi_{2}\right)$ for all $\phi_{1}, \phi_{2} \in \mathfrak{S}_{0}$ and $Z \in \mathfrak{g}_{0}$. Choose $F$ such that $\psi \in E_{F} \mathfrak{W}$. Then if $X \in U$ we have

$$
\begin{aligned}
\lim _{m \rightarrow \infty}\left\|\pi_{0}\left(\exp _{m} X\right) \psi\right\|^{2} & =\lim _{m \rightarrow \infty}\left(\psi, \pi_{0}\left(\exp _{m}(-X)\right) \pi_{0}\left(\exp _{m} X\right) \psi\right) \\
& =\lim _{m \rightarrow \infty}\left(\psi, E_{F} \pi_{0}\left(\exp _{m}(-X)\right) \pi_{0}\left(\exp _{m} X\right) \psi\right) \\
& =\left(\psi, \lim _{m \rightarrow \infty} E_{F} \pi_{0}\left(\exp _{m}(-X)\right) \pi_{0}\left(\exp _{m} X\right) \psi\right) \\
& =\left(\psi, E_{F} \pi(\exp -X) \pi(\exp X) \psi\right)=\left(\psi, E_{F} \psi\right)=\|\psi\|^{2} .
\end{aligned}
$$

This proves that the sequence $\pi_{0}\left(\exp _{m} X\right) \psi$ is bounded in $V$. Moreover, for any $\phi \in \mathfrak{S}_{0}$ we can choose a finite subset $F$ of $\Omega$ such that $\phi \in E_{F} \mathfrak{S}$ and therefore

$$
\lim _{m \rightarrow \infty}\left(\phi, \pi_{0}\left(\exp _{m} X\right) \psi\right)=\lim _{m \rightarrow \infty}\left(\phi, E_{F} \pi_{0}\left(\exp _{m} X\right) \psi\right)=\left(\phi, E_{F} \pi(\exp X) \psi\right) .
$$

Since $\mathfrak{S}_{0}$ is dense in $V$, it follows that the sequence $\pi_{0}\left(\exp _{m} X\right) \psi$ is weakly convergent in $V$. Let $A(X) \psi$ denote the weak limit of this sequence in $V$. Then it is obvious that $A(X)$ is a linear operator with domain $\mathfrak{S}_{0}$. We shall now show that $A(X)$ is bounded. Let $\phi \in \mathfrak{W}_{0}$. Then

$$
(\phi, A(X) \psi)=\lim _{n \rightarrow \infty}\left(\phi, \pi_{0}\left(\exp _{n} X\right) \psi\right) .
$$

Hence $|(\phi, A(X) \psi)| \leqq\|\phi\| \lim _{n \rightarrow \infty}\left\|\pi_{0}\left(\exp _{n} X\right) \psi\right\|=\|\phi\|\|\psi\|$ as we have seen above. Since $\mathfrak{S}_{0}$ is dense in $V$ it follows that

$$
|(\phi, A(X) \psi)| \leqq\|\phi\|\|\psi\|
$$

for all $\phi \in V$ and therefore $\|A(X) \psi\| \leqq\|\psi\|$. As this is true for all $\psi \in \mathfrak{S}_{0}$, $A(X)$ is bounded and therefore it may be extended in a unique manner to a bounded linear operator on $V$. We shall denote this extension again by $A(X)$. Then it is clear that

$$
\|A(X)\|=\sup _{\|\psi\| \leqq 1}\|A(X) \psi\| \leqq 1 \quad(\psi \in V) .
$$

We now claim that $A(X)$ is a unitary operator. For if $X, Y \in U$ and $\phi, \psi \in \mathfrak{S}_{0}$,

$$
(\phi, A(X) A(Y) \psi) \doteq\left(A^{*}(X) \phi, A(Y) \psi\right)
$$

where $A^{*}(X)$ is the adjoint of $A(X)$. Therefore

$$
\begin{aligned}
(\phi, A(X) A(Y) \psi) & =\lim _{n \rightarrow \infty}\left(A^{*}(X) \phi, \pi_{0}\left(\exp _{n} Y\right) \psi\right) \\
& =\lim _{n \rightarrow \infty}\left(\phi, A(X) \pi_{0}\left(\exp _{n} Y\right) \psi\right) \\
& =\lim _{n \rightarrow \infty}\left(\lim _{m \rightarrow \infty}\left(\phi, \pi_{0}\left(\exp _{m} X\right) \pi_{0}\left(\exp _{n} Y\right) \psi\right)\right.
\end{aligned}
$$


since $\pi_{0}\left(\exp _{n} Y\right) \in \mathfrak{S}_{0}$. Now choose $F$ such that $\phi \in E_{F} \mathfrak{g}$. Then we have seen above that

$$
\left(\phi, E_{F} \pi(\exp X) \pi(\exp Y) \psi\right)=\lim _{m \rightarrow \infty, n \rightarrow \infty}\left(\phi, \pi_{0}\left(\exp _{m} X\right) \pi_{0}\left(\exp _{n} Y\right) \psi\right) .
$$

Therefore

$$
(\phi, A(X) A(Y) \psi)=\left(\phi, E_{F} \pi(\exp X) \pi(\exp Y) \psi\right) .
$$

In particular if we put $Y=-X$ we get

$$
(\phi, A(X) A(-X) \psi)=\left(\phi, E_{F} \psi\right)=(\phi, \psi) .
$$

Since $A(X) A(-X)$ is a bounded operator and since $\mathfrak{S}_{0}$ is dense in $\mathfrak{S}$, we conclude that $A(X) A(-X)=I$ where $I$ is the unit operator. Replacing $X$ by $-X$ we obtain $A(-X) A(X)=I$. This shows that $A(X)$ is regular (i.e. it has a two-sided bounded inverse). Moreover,

$$
\|\psi\|=\|A(-X) A(X) \psi\| \leqq\|A(X) \psi\| \leqq\|\psi\|
$$

since $\|A(-X)\|$ and $\|A(X)\|$ are both $\leqq 1$. Therefore $\|A(X) \psi\|=\|\psi\|$ and this proves that $A(X)$ is unitary.

For any $\mathfrak{D} \in \Omega$ let $E_{\mathfrak{D}}^{\prime}$ denote the orthogonal projection of $V$ on $\mathfrak{E}_{\mathfrak{D}}$. Since $\mathfrak{S}_{0}$ is dense in $V$, it is clear that for any $\psi \in V$ the series $\sum \sum_{\mathbb{E} \in \Omega} E_{\mathscr{D}}^{\prime} \psi$ converges to $\psi$ in $V$. For any finite subset of $F$ of $\Omega$ put $E_{F}^{\prime}=\sum_{D \in F} E_{F}^{\prime}$. It is obvious that the operators $E_{F}^{\prime}$ and $E_{F}$ coincide on $\mathfrak{S}_{0}$. We shall now prove that if $X_{1}, \cdots, X_{r} \in U$ and $\psi \in \mathfrak{E}_{0}$ then

$$
E_{F}^{\prime} A\left(X_{1}\right) A\left(X_{2}\right) \cdots A\left(X_{r}\right) \psi=E_{F} \pi\left(\exp X_{1}\right) \pi\left(\exp X_{2}\right) \cdots \pi\left(\exp \dot{X_{r}}\right) \psi
$$

for any finite subset $F$ of $\Omega$. First notice that since strong and weak convergence in $E_{F}^{\prime} V$ are the same,

$$
E_{F}^{\prime} A\left(X_{1}\right) \psi=\lim _{n \rightarrow \infty} E_{F} \pi_{0}\left(\exp _{n} X_{1}\right) \psi=E_{F} \pi\left(\exp X_{1}\right) \psi
$$

and therefore our statement is true if $r=1$. So we may assume $r \geqq 2$ and use induction on $r$. Put $\phi=\pi\left(\exp X_{2}\right) \cdots \pi\left(\exp X_{r}\right) \psi$ and $\phi^{\prime}=A\left(X_{2}\right) \cdots$ $A\left(X_{r}\right) \psi$. Then if $F_{1}$ is any finite subset of $\Omega$ it follows from our induction hypothesis that

$$
E_{F_{1}}^{\prime} \phi^{\prime}=E_{F_{1}} \phi=\phi_{F_{1}} \text { (say). }
$$

Moreover $\phi_{F_{1}} \in E_{F_{1}} \mathfrak{S} \subset \mathfrak{S}_{0}$, and therefore

$$
E_{F}^{\prime} A\left(X_{1}\right) \phi_{F_{1}}=E_{F} \pi\left(\exp X_{1}\right) \phi_{F} .
$$

Since $\psi$ is well-behaved in $\mathfrak{G}$, the same holds for $\phi$. Therefore from Lemma 31 the series $\sum_{\mathbb{D} \in \Omega} E_{\mathbb{D} \phi}$ converges to $\phi$ in $\mathfrak{S}$. On the other hand, we have seen 
above that $\sum_{\mathscr{D} \in \Omega} E_{\mathfrak{D}}^{\prime} \phi^{\prime}$ converges to $\phi^{\prime}$ in $V$. Therefore, since $E_{F}^{\prime} A\left(X_{1}\right)$ and $E_{F} \pi\left(\exp X_{1}\right)$ are bounded operators in $V$ and $\mathfrak{S}$ respectively and since $\phi_{F_{1}}=\sum_{\mathfrak{D} \in F_{1}} E_{\mathfrak{D} \phi}=\sum_{\mathfrak{D} \in F_{1}} E_{\mathfrak{D}}^{\prime} \phi^{\prime}$, it follows from the above result that

$$
E_{F}^{\prime} A\left(X_{1}\right) \phi^{\prime}=E_{F} \pi\left(\exp X_{1}\right) \phi
$$

and this proves our assertion.

Now suppose $X_{1}, \cdots, X_{r}$ is a finite set of elements in $U$ such that $\exp X_{1} \cdots \exp X_{r}=1$. Then for any $\phi, \psi \in \mathfrak{W}_{0}$,

$$
\begin{aligned}
\left(\phi, A\left(X_{1}\right) A\left(X_{2}\right) \cdots A\left(X_{r}\right) \psi\right) & =\left(\phi, E_{F}^{\prime} A\left(X_{1}\right) \cdots A\left(X_{r}\right) \psi\right) \\
& =\left(\phi, E_{F} \pi\left(\exp X_{1}\right) \cdots \pi\left(\exp X_{r}\right) \psi\right) \\
& =\left(\phi, E_{F} \psi\right)=(\phi, \psi)
\end{aligned}
$$

where $F$ is so chosen that $\phi \in E_{F} \mathfrak{E}$. Since $\mathfrak{S}_{0}$ is dense in $V$ and since $A\left(X_{1}\right) A\left(X_{2}\right) \cdots A\left(X_{r}\right)$ is a bounded operator in $V$, this proves that $A\left(X_{1}\right) A\left(X_{2}\right) \cdots A\left(X_{r}\right)=I$.

We know that every $x \in G$ can be written in the form $x=\exp X_{1} \exp X_{2} \cdots$ $\exp X_{r}\left(X_{i} \in U, 1 \leqq i \leqq r\right)$. Then if we put $\sigma(x)=A\left(X_{1}\right) \cdots A\left(X_{r}\right)$ it is clear from what we have proved above that $\sigma(x)$ depends only on $x$ and not on the choice of $X_{1}, \cdots, X_{r}$ and $\sigma(x y)=\sigma(x) \sigma(y)$. Moreover $\sigma(x)$ is obviously a unitary operator and $\sigma(1)=I$. Hence it follows from well known arguments (see Godement $[7(\mathrm{a})]$ ) that in order to show that $\sigma$ is a representation of $G$ on $V$ it is sufficient to prove that $\lim _{x \rightarrow 1}(\phi, \sigma(x) \psi)=(\phi, \psi)$ for any two elements $\phi, \psi \in V$. First suppose $\phi, \psi \in \mathfrak{W}_{0}$. Choose $F$ so that $\phi \in E_{F} \mathfrak{W}$. Then

$$
E_{F}^{\prime} A(X) \psi=E_{F} \pi(\exp X) \psi
$$

and therefore

$$
(\phi, \sigma(\exp X) \psi)=\left(\phi, E_{F} \pi(\exp X) \psi\right) .
$$

Now as $X \rightarrow 0, \pi(\exp X) \psi \rightarrow \psi$ and since $E_{F}$ is a bounded operator on $\mathfrak{E}$, $E_{F} \pi(\exp X) \psi \rightarrow E_{F} \psi$. Hence

$$
\lim _{X \rightarrow 0}(\phi, \sigma(\exp X) \psi)=\left(\phi, E_{F} \psi\right)=(\phi, \psi) .
$$

Now if we take into account the fact that $\mathfrak{W}_{0}$ is dense in $V$ and $\sigma(x)$ is unitary, it follows immediately that $\lim _{x \rightarrow 1}(\phi, \sigma(x) \psi)=(\phi, \psi)$ for all $\phi, \psi \in V$. Therefore $\sigma$ is a unitary representation of $G$ on $V$. Moreover, it is obvious that for any $u \in K$ the operators $\sigma(u)$ and $\pi(u)$ coincide on $\mathfrak{S}_{0}$ and therefore since $\pi$ is a permissible representation and since $\mathfrak{S}_{0}$ is dense in $V, \sigma$ is also permissible. Hence it follows from Lemmas 30 and 34 that $V_{\mathfrak{D}}=\mathfrak{S}_{\mathfrak{D}}$ and every element in $\mathfrak{S}_{0}$ is well-behaved under $\sigma$. Let $X \in U$ and $\phi, \psi \in \mathfrak{S}_{0}$. Then

$$
(\phi, \sigma(\exp X) \psi)=\left(\phi, E_{F}^{\prime} \sigma(\exp X) \psi\right)=\left(\phi, E_{F} \pi(\exp X) \psi\right)
$$


where $F$ is so chosen that $\phi \in E_{F} \mathfrak{W}$. Therefore

$$
\begin{aligned}
\lim _{t \rightarrow 0} \frac{1}{t}(\phi, \sigma(\exp t X) \psi-\psi) & =\lim _{t \rightarrow 0} \frac{1}{t}\left(\phi, E_{F}(\pi(\exp t X) \psi-\psi)\right) \\
& =\left(\phi, E_{F} \pi_{0}(X) \psi\right)=\left(\phi, \pi_{0}(X) \psi\right) .
\end{aligned}
$$

Since $\mathfrak{S}_{0}$ is dense in $V$ and since $\psi$ is well-behaved under $\sigma$ this shows that

$$
\lim _{t \rightarrow 0} \frac{1}{t}(\sigma(\exp t X) \psi-\psi)=\pi_{0}(X) \psi .
$$

Therefore the representation of $\mathfrak{B}$ on $\mathfrak{E}_{0}$ corresponding to $\sigma$ coincides with $\pi_{0}$, and this proves that $\pi$ and $\sigma$ are infinitesimally equivalent. Since $\pi$ is irreducible, it follows from Theorem 5 that $\mathfrak{S}_{0}$ is irreducible under $\pi_{0}$ and therefore $\sigma$ is also irreducible.

Finally, if $\tau$ is any permissible unitary representation of $G$ on a Hilbert space $\Re$ which is infinitesimally equivalent to $\pi$ it is clear that $\tau$ is quasisimple and $\operatorname{dim} \Re_{D}=\operatorname{dim} \mathfrak{E}_{\mathbb{D}}<\infty$. Therefore, from Theorem $5, \tau$ is irreducible. Since $\sigma$ and $\tau$ are irreducible unitary representations which are infinitesimally equivalent, it follows from Theorem 8 that they are equivalent.

12. Explicit construction of some representations. We shall now give a method for constructing a certain class $\left.{ }^{13}\right)$ of representations of $G$. This is a generalisation of a method given by Gelfand and Naimark [6(a), (b)] in the case when $G$ is a complex semi-simple Lie group (see also [8(e), Part IV]).

First we compute the Haar measure of $G$ in terms of the Haar measures $\left({ }^{14}\right)$ of $K, A_{+}$, and $N$, respectively. The group $A_{+}$being simply-connected, we denote by $\log h\left(h \in A_{+}\right)$the unique element $H \in \mathfrak{h}_{\mathfrak{p}_{0}}$ such that $h=\exp H$. Moreover set $\rho=2^{-1} \sum_{\alpha \in P} \alpha$ where the notation is that of Lemma 5.

Lemma 35. Let $d x, d u, d h$, and $d n$ denote the elements of Haar measures on $G, K, A_{+}$, and $N$ respectively. Then

$$
d x=e^{2 \rho(\log h)} d u d h d n \quad\left(x=u h n, u \in K, h \in A_{+}, n \in N\right) .
$$

Let $S=A_{+} N$. Then in the notation of Lemma $27 d x=\mu(s) d u d s(x=u s$, $u \in K, s \in S$ where $\mu(s)=\operatorname{det} \operatorname{Ad}_{s}(s) / \operatorname{det} \operatorname{Ad}(s)$ and $d s$ is the element of the left-invariant measure on $S$ ). But since $G$ is semi-simple, det $\operatorname{Ad}(x)=1$ for all $x \in G$. Hence $\mu(s)=\operatorname{det} \operatorname{Ad}_{s}(s)$. Moreover, by applying the same lemma to $S=A_{+} N$ we get

$$
d s=\frac{\operatorname{det} \operatorname{Ad}_{N}(n)}{\operatorname{det} \operatorname{Ad}_{s}(n)} d h d n \quad\left(s=h n, h \in A_{+}, n \in N\right)
$$

(13) It seems very likely that this method gives all irreducible quasi-simple representations within infinitesimal equivalence (see [8(d)]).

(14) For the meaning of the various symbols see the beginning of Part III ( $\$ 8$ ). 
where $n \rightarrow \operatorname{Ad}_{N}(n)$ is the adjoint representation of $N$. Since $N$ is nilpotent, $\operatorname{det} \operatorname{Ad}_{N}(n)=1$ and therefore

$$
d x=\operatorname{det} \operatorname{Ad}_{S}(h) d u d h d n=e^{\mathrm{sp}(\sigma(\log h))} d u d h d n=e^{2 \rho(\log h)} d u d h d n
$$

in the notation of Lemma 5 .

Let $u \rightarrow u^{*}$ denote the natural mapping of $K$ on $K^{*}=K / D \cap Z$ and let $d u^{*}$ denote the element of Haar measure of $K^{*}$ so normalised that $\int_{K^{*}} d u^{*}=1$. Let $L_{2}\left(K^{*}\right)$ denote the Hilbert space of all square integrable functions $f$ on $K^{*}$. We shall now define a class of representations of $G$ on $L_{2}\left(K^{*}\right)$. For any $x \in G$ and $u \in K$ write $x u=u_{x} h(x, u) n(x, u)\left(u_{x} \in K, h(x, u) \in A_{+}, n(x, u) \in N\right)$. Moreover, let $\Gamma(u)$ denote the unique element in $c_{0}$ such that $u \exp (-\Gamma(u))$ lies in the commutator group $K^{\prime}$ of $K$. Then it is easy to verify that $\left(u_{x}\right)^{*}, \Gamma\left(u_{x}\right)$ $-\Gamma(u)$, and $h(x, u)$ depend only on $x$ and $u^{*}$. Hence we may write them as $u_{x}^{*}, \Gamma\left(x, u^{*}\right)$, and $h\left(x, u^{*}\right)$ respectively. It is obvious that these elements depend continuously on $\left(x, u^{*}\right)$. Put $H\left(x, u^{*}\right)=\log h\left(x, u^{*}\right)$.

Lemma 36. If $x, y \in G$, then $u_{y x}^{*}=\left(u_{x}^{*}\right)_{y}$ and

$$
H\left(y x, u^{*}\right)=H\left(y, u_{x}^{*}\right)+H\left(x, u^{*}\right), \quad \Gamma\left(y x, u^{*}\right)=\Gamma\left(y, u_{x}^{*}\right)+\Gamma\left(x, u^{*}\right)
$$

For we have $x u=u_{x} h(x, u) n_{1}\left(n_{1} \in N\right)$ and therefore

$$
\begin{aligned}
y x u & =y u_{x} h(x, u) n_{1}=\left(u_{x}\right)_{y} h\left(y, u_{x}\right) n_{2} h(x, u) n_{1} \quad\left(n_{2} \in N\right) \\
& =\left(u_{x}\right)_{y} h\left(y, u_{x}\right) h(x, u) n_{2}^{\prime} n_{1}
\end{aligned}
$$

where $n_{2}^{\prime}=(h(x, u))^{-1} n_{2} h(x, u) \in N$. Therefore

$$
(u)_{y x}=\left(u_{x}\right)_{y}, \quad h(y x, u)=h\left(y, u_{x}\right) h(x, u)
$$

and

$$
\begin{aligned}
\Gamma(y x, u) & =\Gamma\left(u_{y x}\right)-\Gamma(u)=\Gamma\left(\left(u_{x}\right)_{y}\right)-\Gamma\left(u_{x}\right)+\Gamma\left(u_{x}\right)-\Gamma(u) \\
& =\Gamma\left(y, u_{x}\right)+\Gamma(x, u) .
\end{aligned}
$$

The statements of the lemma now follow immediately.

Let $\nu$ and $\mu$ be (complex-valued) linear functions on $\mathfrak{h}_{\mathfrak{p}_{0}}$ and $\mathfrak{c}_{0}$ respectively. Then we define a representation $\pi_{\mu, \nu}$ of $G$ on $L_{2}\left(K^{*}\right)$ as follows. For any $f \in L_{2}\left(K^{*}\right), \pi_{\mu, \nu}(x) f(x \in G)$ is the function $g$ given by

$$
g\left(u^{*}\right)=e^{-\mu\left(\Gamma\left(x-1 u^{*}\right)\right)} e^{-\nu^{\prime}\left(H\left(x-1, u^{*}\right)\right)} f\left(u_{x-1}^{*}\right)
$$

where $\nu^{\prime}=\nu+2 \rho$. First of all, we have to verify that $g \in L_{2}\left(K^{*}\right)$. Notice that if $y=u h n\left(u \in K, h \in A_{+}, n \in N\right)$ then

$$
x y=u_{x} h(x, u) h\left(h^{-1} n(x, u) h n\right) .
$$

Hence if $x$ is fixed,

$$
e^{2 \rho(\log h)} d u d h d n=d y=d(x y)=e^{2 \rho(\log (h(x, u) h))} d u_{x} d h d n
$$


since $d(h(x, u) h)=d h$ for fixed $x$ and $u$ and $d\left(\left(h^{-1} n(x, u) h\right) n\right)=d n$ for fixed $x, u$, and $h$. This shows that $d u=e^{2 \rho(H(x, u))} d u_{x}$ and from this it follows immediately that

$$
d u^{*}=e^{2 \rho\left(H\left(x, u^{*}\right)\right)} d u_{x}^{*}
$$

since $K$ and $K^{*}$ are locally isomorphic. Hence

$$
\int_{K^{*}}\left|g\left(u^{*}\right)\right|^{2} d u^{*}=\int_{K^{*}}\left|g\left(u_{x}^{*}\right)\right|^{2} d u_{x}^{*}=\int_{K^{*}}\left|g\left(u_{x}^{*}\right) e^{-\rho\left(H\left(x, u^{*}\right)\right)}\right|^{2} d u^{*} .
$$

Now $\Gamma\left(x^{-1}, u_{x}^{*}\right)=-\Gamma\left(x, u^{*}\right)$ and $H\left(x^{-1}, u_{x}^{*}\right)=-H\left(x, u^{*}\right)$ from Lemma 36. Therefore

$$
g\left(u_{x}^{*}\right) e^{-\rho\left(H\left(x, u^{*}\right)\right)}=e^{\mu\left(\Gamma\left(x, u^{*}\right)\right)} e^{(v+\rho)\left(H\left(x, u^{*}\right)\right)} f\left(u^{*}\right) .
$$

The function $\left|e^{\mu\left(\Gamma\left(x, u^{*}\right)\right)} e^{(\nu+\rho)\left(B\left(x, u^{*}\right)\right)}\right|$ being continuous is bounded on the compact set $K^{*}$ and therefore if $M_{x}$ is a bound for it

$$
\int_{K^{*}}\left|g\left(u^{*}\right)\right|^{2} d u^{*} \leqq M_{x}^{2} \int\left|f\left(u^{*}\right)\right|^{2} d u^{*}<\infty .
$$

Hence $g \in L_{2}\left(K^{*}\right)$ and $\left|\pi_{\mu, \nu}(x)\right| \leqq M_{x}$, so that $\pi_{\mu, \nu}(x)$ is bounded. Moreover, it follows easily from Lemma 36 that $\pi_{\mu, \nu}(y) \pi_{\mu, \nu}(x)=\pi_{\mu, \nu}(y x)(x, y \in G)$. Therefore, it only remains to verify the continuity of $\pi_{\mu, v}$. Let $U$ be a compact neighbourhood of 1 in $G$. Then

$$
\sup _{x \in U, u^{*} \in K^{*}}\left|e^{\mu\left(\Gamma\left(x, u^{*}\right)\right)} e^{(v+\rho)\left(H\left(x, u^{*}\right)\right)}\right|=M_{U}<\infty
$$

since $e^{\mu\left(\Gamma\left(x, u^{*}\right)\right)} e^{(v+\rho)\left(B\left(x, u^{*}\right)\right)}$ is a continuous function on $G \times K^{*}$. Hence $\left|\pi_{\mu, \nu}(x)\right|$

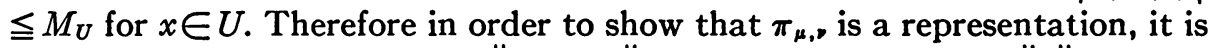
sufficient to prove that $\lim _{x \rightarrow 1}\left\|\pi_{\mu, n} f-f\right\|=0\left(f \in L_{2}\left(K^{*}\right)\right)$ where $\|\cdot\|$ denotes the usual norm in $L_{2}\left(K^{*}\right)$. Given $\epsilon>0$, choose a continuous function $g$ on $K^{*}$ such that $\|f-g\| \leqq \epsilon$. Then it is clear that we can find a neighbourhood $V$ of 1 in $G(V \subset U)$ such that $\left|\pi_{\mu, \nu}(x) g-g\right| \leqq \epsilon(x \in V)$. Hence

$$
\left\|\pi_{\mu, \nu}(x) g-g\right\|^{2}=\int_{K^{*}}\left|\pi_{\mu, \nu}(x) g-g\right|^{2} d u^{*} \leqq \epsilon^{2} \quad(x \in V)
$$

and

$$
\begin{aligned}
\left\|\pi_{\mu, \nu}(x) f-f\right\| & =\left\|\pi_{\mu, \nu}(x)(f-g)-(f-g)+\left(\pi_{\mu, \nu}(x) g-g\right)\right\| & \\
& \leqq\left|\pi_{\mu, \nu}(x)\right| \epsilon+\epsilon+\epsilon \leqq(M,+2) \epsilon & (x \in V) .
\end{aligned}
$$

This proves that $\pi$ is a representation of $G$.

Let $\lambda$ be the left regular representation of $K^{*}$ on $L_{2}\left(K^{*}\right)$ so that $\lambda\left(v^{*}\right) f=g$ $\left(f \in L_{2}\left(K^{*}\right), v^{*} \in K^{*}\right)$ where $g\left(u^{*}\right)=f\left(v^{*-1} u^{*}\right)$. Then we check easily that 


$$
\lambda\left(u^{*}\right)=e^{-\mu(\Gamma(u))} \pi_{\mu, \nu}(u)
$$

Let $\mathfrak{S}=L_{2}\left(K^{*}\right)$ and let $\mathfrak{S C D}_{\mathfrak{D}}(\mathfrak{D} \in \Omega)$ denote the set of all elements in $\mathfrak{E}$ which transform under $\pi_{\mu, \nu}(K)$ according to $\mathfrak{D}$. Then if $\mathfrak{E}_{\mathfrak{D}} \neq\{0\}$, it is clear that there exists an equivalence class $\mathfrak{D}^{*}$ of finite-dimensional irreducible representations of $K^{*}$ such that every element of $\mathfrak{S}_{\mathfrak{D}}$ transforms under $\lambda$ according to $\mathfrak{D}^{*}$. The degrees of the representations in $\mathfrak{D}$ and $\mathfrak{D}^{*}$ are obviously the same. Hence if $d(D)$ denotes this degree, it follows from well known results on compact groups that $\operatorname{dim} \mathfrak{W}_{\mathfrak{D}} \leqq(d(\mathfrak{D}))^{2}$. Moreover $\pi_{\mu, \nu}$ is permissible since $\pi_{\mu, \nu}(x)=e^{\mu(\Gamma(x))} \pi_{\mu, \nu}(1)$ if $x \in D \cap Z$. Therefore from Lemma 34 every element in $\sum_{\mathbb{D} \in \Omega} \mathfrak{S}_{\mathbb{D}}$ is well-behaved under $\pi_{\mu, v}$. We have seen above that if $f \in L_{2}\left(K^{*}\right)$

$$
\int_{K^{*}}\left|\pi_{\mu, \nu}(x) f\right|^{2} d u^{*}=\int_{K^{*}}\left|e^{\mu\left(\Gamma\left(x, u^{*}\right)\right)} e^{(\nu+\rho)\left(H\left(x, u^{*}\right)\right)} f\left(u^{*}\right)\right|^{2} d u^{*} .
$$

Therefore if the linear functions $\mu$ and $\nu+\rho$ take purely imaginary values on $\mathfrak{c}_{0}$ and $\mathfrak{h}_{\mathfrak{p}_{0}}$, the representation is unitary

Let $M$ be the analytic subgroup of $G$ corresponding to the algebra $\left.{ }^{(15}\right) \mathfrak{m}_{0}$ $=\mathfrak{m} \cap g_{0}$ and let $x \rightarrow x^{*}$ denote the natural mapping of $G$ on $G / D \cap Z=G^{*}$. Since $D \subset K$, the mapping $\left(u^{*}, a, n\right) \rightarrow u^{*} a^{*} n^{*}\left(u^{*} \in K^{*}, a \in A_{+}, n \in N\right)$ is a homeomorphism of $K^{*} \times A_{+} \times N$ onto $G^{*}$. Therefore $A_{+}^{*}$ is closed in $G^{*}$. Since $\mathfrak{m}_{0}$ is the centraliser of $\mathfrak{h}_{\mathfrak{p}_{0}}$ in $\mathfrak{l}_{0}$ (Lemma 4), $M^{*}$ is the component of identity of the centraliser of $A_{+}^{*}$ in $K^{*}$. Therefore $M^{*}$ is closed and hence compact.

Let $\tau$ denote the right regular representation of $K^{*}$ so that

$$
\left(\tau\left(u^{*}\right) f\right)\left(v^{*}\right)=f\left(v^{*} u^{*}\right) \quad\left(f \in L_{2}\left(K^{*}\right) ; u^{*}, v^{*} \in K^{*}\right) .
$$

Lemma 37. Let $m \in M$ and $x \in G$. Then $\tau\left(m^{*}\right)$ commutes with $\pi_{\mu, \nu}(x)$.

Let $u \in K$. Then $x u=u_{x} h(x, u) n(n \in N)$. From Lemmas 4 and 5, $n^{\prime}$ $=m^{-1} n m \in N$ and $h(x, u) m=m h(x, u)$. Hence $x u m=u_{x} m h(x, u) n^{\prime}$ and therefore $(u m)_{x}=u_{x} m, h(x, u m)=h(x, u)$ and $\Gamma(x, u m)=\Gamma(x, u)$. Our assertion follows immediately from these facts.

Let $\sigma$ be an irreducible representation of $M$ on a finite-dimensional space $V$. It is clear that we can choose $\mu$ such that $\sigma(\exp \Gamma)=e^{\mu(\Gamma)} \sigma(1)$ if $\exp \Gamma$ $\in M \cap Z\left(\Gamma \in \mathfrak{c}_{0}\right)$. Put $\sigma^{*}\left(m^{*}\right)=e^{-\mu(\Gamma(m))} \sigma(m)(m \in M)$. Then $\sigma^{*}$ is an irreducible representation of $M^{*}$ on $V$. Now it follows from well known results (see [17]) on compact groups that we can choose a continuous function $\phi$ on $K^{*}$ such that the linear space spanned by the functions $\tau\left(m^{*}\right) \phi\left(m^{*} \in M^{*}\right)$ is of finite dimension and the representation of $M^{*}$ induced on it under $\tau$ is irreducible and dual to $\sigma^{*}$. Let $\mathfrak{S}_{\phi}$ be the smallest closed subspace of $L_{2}\left(K^{*}\right)$ containing $\phi$ which is invariant under $\pi_{\mu, \nu}$. Let $\pi_{\mu, \nu}^{\prime}$ denote the representation

(is) See $\$ 2$ (Lemma 4) for the definition of $\mathfrak{m}$. 
of $G$ induced on $\mathfrak{W}_{\phi}$ under $\pi_{\mu, \nu}$. Then it can be shown that $\pi_{\mu, \nu}^{\prime}$ is quasi-simple. Let $\delta$ be the equivalence class of the representation $\sigma$. Since $\mathfrak{m}$ is reductive in $\mathfrak{l}$ (Lemma 5), every class $\mathfrak{D} \in \Omega$ is fully reducible with respect to $M$. Let $(D: \delta)$ denote the number of times $\delta$ occurs in this reduction. Then it follows from the Frobenius reciprocity relation (see $\left[17\right.$, p. 83]) that $\operatorname{dim}\left(\mathfrak{S}_{\phi}\right)_{\mathbb{D}}$ $\leqq d(\mathfrak{D})(\mathfrak{D}: \delta)$.

We shall return to a more detailed study of these representations of $G$ in another paper.

\section{REFERENCES}

1. S. Banach, Leçons sur les éspaces linéares, Warsaw, 1932.

2. G. Birkhoff, Ann. of Math. vol. 38 (1937) pp. 526-532.

3. E. Cartan, J. Math. Pures Appl. vol. 8 (1929) pp. 1-33.

4. C. Chevalley, Theory of Lie groups, Princeton University Press, 1946.

5. L. Gårding, Proc. Nat. Acad. Sci. U.S.A. vol. 33 (1947) pp. 331-332.

6. I. M. Gelfand and M. A. Naimark, (a) Doklady Akademii Nauk SSSR. N.S. vol. 63 (1948) pp. 225-228.
(b) Trudi Mat. Inst. Steklova vol. 36 (1950).

7. R. Godement, (a) Trans. Amer. Math. Soc. vol. 63 (1948) pp. 1-84.

- (b) J. Math. Pures Appl. vol. 30 (1951) pp. 1-110.

-, (c) On spherical functions, Trans. Amer. Math. Soc. vol. 73 (1952) pp. 496-566.

8. Harish-Chandra, (a) Ann. of Math. vol. 50 (1949) pp. 900-915.

(b) Proc. Amer. Math. Soc. vol. 1 (1950) pp. 205-210.

(c) Proc. Nat. Acad. Sci. U.S.A. vol. 37 (1951) pp. 170-173:

(d) Proc. Nat. Acad. Sci. U.S.A. vol. 37 (1951) pp. 362-365, 366-369, 691-694.

(e) Trans. Amer. Math. Soc. vol. 70 (1951) pp. 28-96.

9. K. Iwasawa, Ann. of Math. vol. 50 (1949) pp. 507-557.

10. N. Jacobson, Ann. of Math. vol. 36 (1935) pp. 875-881.

11. L. Koszul, Bull. Soc. Math. France vol. 78 (1950) pp. 65-127.

12. F. I. Mautner, Ann. of Math. vol. 52 (1950) pp. 528-556.

13. G. D. Mostow, Bull. Amer. Math. Soc. vol. 55 (1949) pp. 969-980.

14. F. J. Murray and J. von Neumann, Ann. of Math. vol. 37 (1936) pp. 116-229.

15. I. E. Segal, Proc. Amer. Math. Soc. vol. 3 (1952) pp. 13-15.

16. B. L. van der Waerden, Moderne Algebra, Berlin, Springer, 1937. 1940.

17. A. Weil, L'integration dans les groupes topologiques et ses applications, Paris, Hermann,

\section{Columbia University,} NEW YoRK, N Y. 